\title{
Sensitivity Enhanced NMR
}

\author{
Dissertation \\ for the award of the degree \\ Doctor rerum naturalium \\ of the University of Göttingen
}

within the doctoral program Physics of Biological and Complex Systems of the Georg-August University School of Science (GAUSS)

submitted by

Philip Lottmann

from Hildesheim

Göttingen 2013 


\section{Thesis Committee}

Prof. Dr. Christian Griesinger

NMR based Structural Biology, Max Planck Institute for Biophysical Chemistry, Göttingen

Prof. Dr. Marina Bennati

Electron Spin Resonance Spectroscopy, Max Planck Institute for Biophysical Chemistry, Göttingen

Prof. Dr. Andreas Janshoff

Institute for Physical Chemistry, University of Göttingen

\section{Members of the Examination Board}

Referee:

Prof. Dr. Christian Griesinger

NMR based Structural Biology, Max Planck Institute for Biophysical Chemistry,

Göttingen

$2^{\text {nd }}$ Referee:

Prof. Dr. Marina Bennati

Electron Spin Resonance Spectroscopy, Max Planck Institute for Biophysical Chemistry, Göttingen

\section{Further Members of the Examination Board}

Dr. Frank Engelke

Probe Development, Bruker BioSpin GmbH, Rheinstetten

Dr. Adam Lange

NMR based Structural Biology, Max Planck Institute for Biophysical Chemistry,

Göttingen

Dr. Martin Kollmar

NMR based Structural Biology, Max Planck Institute for Biophysical Chemistry,

Göttingen

Prof. Dr. Andreas Janshoff

Institute for Physical Chemistry, University of Göttingen

Date of oral examination: 28.01.2014 




\section{Affidavit}

I hereby declare that I wrote this thesis Sensitivity Enhanced NMR on my own and without the use of any other than the cited sources and tools. 



\section{Acknowledgments}

Firstly, I would like to express my sincere gratitude to my supervisor Prof. Dr. Christian Griesinger for the opportunity to work on this very interesting project as well as the opportunities, which came with it. Furthermore, I would like to thank him for the constructive discussions and his patience.

I am eternally grateful to Thorsten Marquardsen for his unwavering and limitless commitment to the project as well as his friendship.

I am much obliged to Dr. Alexander Krahn and Andreas Tavernier for their instructions, quick help and support.

In addition, I very much appreciate the feedback offered by Prof. Dr. Marina Bennati and Dr. Frank Engelke throughout my thesis.

I would like to thank Dr. Stefan Becker and Sebastian Wolf for providing the Ubiquitin sample as well as Andrei Leonov for providing the polarizer I used for all my experiments.

Furthermore, I greatly benefited from Frank Delagio by introducing me to the macro programming of NMRPipe.

I would like to thank Prof. Dr. Andreas Janshoff for supporting me in my thesis committee.

I am indept to Eibe Dücker and Dr. Stefan Bartoschek for carefully reading my thesis. 
My special thanks to Petra Breiner, who shuttled me to Karlsruhe and beyond.

I would like to thank the whole NMR II department for the nice and friendly atmosphere as well as the Bruker department for probe development for their warm welcome and for putting up with during the lunch breaks.

Dr. Florian Siepel, Dr. David Ban, Dr. Adam Mazure and Dr. Donghan Lee: Thank you for all the helpful discussions!

Furthermore, I am deeply grateful to Dr. Florian Siepel and Birte Gerlach for friendship, support and so much more than I can say.

In the end, I would like to thank my family and all my friends, who supported me throughout my $\mathrm{PhD}$ thesis. 


\section{Abstract}

The topic of this thesis is the investigation of how sensitivity enhanced Nuclear Magnetic Resonance (NMR) spectra of biological systems could be obtained by dynamic nuclear polarization (DNP) under conditions close to their physiological environment in solution. Here, the Overhauser effect is the driving mechanism for DNP, which depends on the diffusion kinetics of the polarizer molecule with an unpaired electron and the nuclei of the target molecule. For this thesis, a liquid state shuttle $\mathrm{DNP}$ spectrometer was chosen, where the nuclei are polarized in a field of $0.34 \mathrm{~T}$ and transferred to a field of $14.09 \mathrm{~T}$ for high resolution NMR detection. Several technical modifications were successively implemented, which increased stability and improved reproducibility of the measurements.

High-field DNP enhancements $\varepsilon_{\text {hf }}$ up to $-2.4\left(\mathrm{H}^{\eta^{2}}\right)$ were measured for the proton signals of L-tryptophan. A general global enhancement factor $\varepsilon_{\text {global }}$ was introduced which includes advantages and disadvantages of the shuttle DNP approach, such as an increased acquisition rate of the DNP experiments and line broadening due to the presence of the radical, respectively. Subsequently, it was applied and adapted to one-dimensional NMR measurements. For this purpose the polarization build-up time and the acquisition time was optimized for the Boltzmann polarization and the DNP measurement to gain a maximal signal-to-noise ratio per unit measurement time based on $T_{1}$ and $T_{2}^{*}$, respectively. With this, an applied global enhancement $\varepsilon_{\text {app }}$ factor of -4.0 for the $\mathrm{H}^{\delta 1}$ of L-tryptophan was measured.

Furthermore, the DNP spin properties of the protons, such as relaxation rates, were measured and compared with each other. The thereby obtained coupling factors implied that the proton accessibility for the polarizer molecule had an important influence on the intermolecular dipolar interaction between the nuclear spins of the target molecule and the electron of the polarizer. It was shown, that this interaction is described best by a model based on translational diffusion. With this model, the 
distance of closest approach was determined for the protons of L-tryptophan. These distances range from 3 to $5 \AA$ corresponding to the accessibility of each respective proton.

In addition, carbon DNP enhancements between -0.3 and -2.5 were measured for deuterated L-tryptophan- $\mathrm{d}_{8},{ }^{15} \mathrm{~N}_{2},{ }^{13} \mathrm{C}_{11}$. Calculations demonstrated that these carbon enhancements were in agreement with the previously calculated distance of closest approach of the proton spins and therefore confirmed the approach of the translational diffusion model, too. In further measurements on protonated Ltryptophan- ${ }^{15} \mathrm{~N}_{2},{ }^{13} \mathrm{C}_{11}$, the three-spin effect was observed for the first time for a solute molecule. This effect, based on a dipolar interaction between the proton, the carbon and the electron spin, caused positive enhancements for all carbons up to 2.3, but the carbonyl carbon with an enhancement of -2.5. These findings are in agreement with an expanded coupling factor, which includes the intramolecular carbon-proton interaction alongside the intermolecular carbon-electron interaction.

In a concluding step, shuttle DNP experiments were conducted on a protein (Ubiquitin-U- ${ }^{15} \mathrm{~N}, \mathrm{U}_{-}{ }^{13} \mathrm{C}$ ). For this purpose, a two dimensional shuttle DNP ${ }^{1} \mathrm{H}_{-}{ }^{13} \mathrm{C}-$ HSQC spectrum was recorded. For the first time, a DNP transfer to the surface of a protein was demonstrated in the liquid state. 


\section{Zusammenfassung}

Das Thema dieser Arbeit ist die Sensitivitätserhöhung der Kernspinmagnetresonanzspektroskopie (NMR-Spektroskopie) für die Anwendung an biologischen Systemen durch dynamische Kernspinpolarisation (DNP). Dementsprechend wurden die experimentellen Bedingungen möglichst ähnlich zu einer physiologischen Umgebung in Lösung gewählt. Unter diesen Voraussetzungen ist der Overhauser-Effekt der zentrale Mechanismus für DNP. Dieser ist von der relativen Diffusion zwischen den Kernspins des Zielmoleküls und dem polarisierenden Molekül, welches ein ungepaartes Elektron aufweist, abhängig. Als experimenteller Ansatz für diese Arbeit wurde ein Shuttle-DNP-Spektrometer mit Proben im flüssigen Zustand ausgewählt. Hierbei wurden die Kernspins bei einem Magnetfeld von 0,34 T polarisiert und für eine hoch auflösende NMR-Detektion in ein Magnetfeld von 14,09 T transferiert. Mehrere technische Anpassungen, welche zu einer Erhöhung der Stabilität und Reproduzierbarkeit der Messungen führten, wurden sukzessiv implementiert.

Für die Signale der Protonen von L-Tryptophan wurde im Hochfeld eine DNP. Verstärkung $\varepsilon_{\mathrm{hf}}$ von bis zu -2,4 $\left(\mathrm{H}^{\eta^{2}}\right)$ gemessen. Darauf aufbauend wurde ein allgemeiner Verstärkungsfaktor $\varepsilon_{\text {global }}$ eingeführt. Dieser beinhaltete sowohl die Vorteile des Shuttle-DNP-Spektrometers, wie beispielsweise die schnellere Aufnahmerate der DNP-Experimente als auch die Nachteile, wie etwa die Linienverbreiterung der Signale durch die Gegenwart des polarisierenden Radikals. Anschließend wurde dieser Faktor schrittweise auf eindimensionale Messungen angewandt und an diese angepasst. Hierfür wurden die Aufbaurate der Polarisation und die Aufnahmezeit der Messungen mit DNP und Boltzmann-Polarisation optimiert, um das maximale Signal-zu-Rauschen-Verhältnis pro Messzeit zu erhalten. Diese Parameter basieren auf $T_{1}$ bzw. $T_{2}^{*}$. Das Ergebnis dieser Schritte war ein angewandter, allgemeiner Verstärkungsfaktor $\varepsilon_{\text {app }}$ von -4.0 für $\mathrm{H}^{\delta 1}$ von L-Tryptophan.

Des Weiteren wurden die Kernspineigenschaften von Protonen für DNP, wie z.B. 
die Relaxationsraten, gemessen und miteinander verglichen. Der daraus abgeleitete Kopplungsfaktor implizierte, dass die intermolekulare, dipolare Wechselwirkung zwischen den Kernspins des Zielmoleküls und dem Elektron des polarisierenden Radikals von der räumlichen Zugänglichkeit der Kernspins beeinflusst wurde. Zudem wurde gezeigt, dass diese Wechselwirkung am besten durch ein Model basierend auf translatorischer Diffusion beschrieben werden konnte. Mit diesem wurde der Abstand der dichtesten Annährung zwischen den Kernspins und dem ungepaartem Elektron bestimmt. Diese Abstände reichen entsprechend der Zugänglichkeit des jeweiligen Protons von 3 bis $5 \AA$.

Darauf aufbauend wurden dieDNP-Verstärkungen für Kohlenstoff gemessen. Für deuteriertes L-Tryptophan- $\mathrm{d}_{8},{ }^{15} \mathrm{~N}_{2},{ }^{13} \mathrm{C}_{11}$ wurden Verstärkungen zwischen -0,3 und $-2,5$ erzielt. Durch weitere Berechnungen wurde gezeigt, dass diese Verstärkungen mit den zuvor berechneten Abständen der dichtesten Annäherung der Protonen übereinstimmten und dadurch den Ansatz des Models der translatorischen Diffusion untermauerten. In weiteren Messungen an protoniertem L-Tryptophan- ${ }^{15} \mathrm{~N}_{2},{ }^{13} \mathrm{C}_{11}$ wurde der Drei-Spin-Effekt erstmalig bei einem gelösten Molekül beobachtet. Dieser Effekt basierte auf der dipolaren Wechselwirkung zwischen den Spins der Protonen, Kohlenstoffkerne und Radikal-Elektronen. Er verursachte positive Signalverstärkungen von bis zu 2,3 für alle Kohlenstoffe außer dem Carbonyl-Kohlenstoff, welcher eine Signalverstärkung von -2,5 aufwies. Diese Ergebnisse waren in Übereinstimmung mit einem erweiterten Kopplungsfaktor, der die intramolekulare Wechselwirkung zwischen Kohlenstoff und Proton neben der zwischen Kohlenstoff und Elektron berücksichtigte.

In einem abschließenden Schritt wurden DNP-Experimente an einem Protein (Ubiquitin-U- ${ }^{15} \mathrm{~N}, \mathrm{U}-{ }^{13} \mathrm{C}$ ) durchgeführt. Zu diesem Zweck wurden zweidimensionale Shuttle-DNP ${ }^{1} \mathrm{H}_{-}{ }^{13} \mathrm{C}-\mathrm{HSQC}$-Spektren aufgenommen. Zum ersten Mal konnte ein DNP-Transfer zu der Oberfläche eines Proteins in Lösung nachgewiesen werden. 




\section{Contents}

Acknowledgments

Abstract III

Zusammenfassung V

$\begin{array}{ll}\text { 1. Introduction } & 1\end{array}$

1.1. General introduction to dynamic nuclear polarization . . . . . . . . 1

1.2. Aims and outline . . . . . . . . . . . . . . . . . 8

1.3. Theory of liquid state shuttle DNP . . . . . . . . . . . . 9

1.3.1. Basics of shuttle Overhauser DNP . . . . . . . . . . . 9

1.3.2. Shuttle DNP . . . . . . . . . . . . . . . 12

1.3.3. Leakage and saturation factor . . . . . . . . . . . . 13

1.3.4. Coupling factor . . . . . . . . . . . . . . . . . 15

2. The DNP shuttle spectrometer with a two center magnet 21

2.1. Overview . . . . . . . . . . . . . . . . . . . 21

2.2. Low field part . . . . . . . . . . . . . . . . 23

2.2.1. Low field plateau . . . . . . . . . . . . . . . . 23 23

2.2.2. DNP probe . . . . . . . . . . . . . . 25

2.3. Sample shuttle system . . . . . . . . . . . . . . 28

2.3.1. Sample shuttle container . . . . . . . . . . . . . . . 28

2.3.2. Shuttle cycle . . . . . . . . . . . . . . . . 30

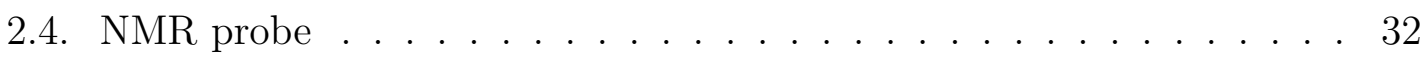

3. Material and Methods

3.1. Chemical reagents and sample preparation ........... 35 
3.2. Sample temperature and microwave power ............ 37

3.3. NMR and DNP experiments . . . . . . . . . . . . . . 38

4. Results and discussion $\quad 43$

4.1. DNP signal enhancement of protons . . . . . . . . . . . . . . 43

4.2. Global proton enhancement . . . . . . . . . . . . . 46

4.2.1. Introducing the global enhancement factor . . . . . . . . . . . . . 46

4.2.2. Applied global enhancement factor . . . . . . . . . . . . 47

4.3. The coupling factor . . . . . . . . . . . . . . . . 55

4.3.1. Calculation of the coupling factor . . . . . . . . . 55

4.3.2. Calculation of the distance of closest approach . . . . . . . . 57

4.4. DNP signal enhancement of carbon-13 . . . . . . . . . . . 63

4.4.1. Carbon enhancement in a two-spin system . . . . . . . . . . 63

4.4.2. Carbon enhancement in a three-spin system . . . . . . . . . 65

4.5. First results on ubiquitin . . . . . . . . . . . . . . . . 70

4.6. Outlook .......................... 75

\begin{tabular}{ll} 
Appendices & 77 \\
\hline
\end{tabular}

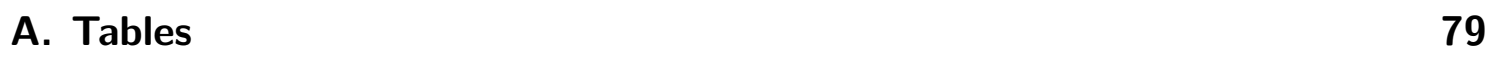

A.1. List of abbreviations . . . . . . . . . . . . . . . 79

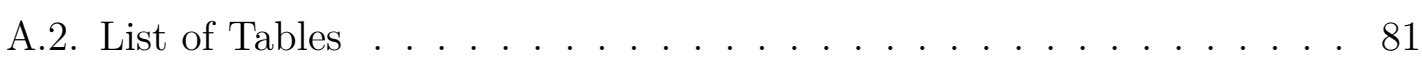

A.3. List of Figures . . . . . . . . . . . . . . . . . 82

\begin{tabular}{l|l} 
B. Literature & 83 \\
\hline
\end{tabular}

C. Pulse programs and scripts $\quad 91$

C.1. Pulse programs ........................... 91

C.2. Scilab script . . . . . . . . . . . . . . . . 112

C.3. NMRPipe scripts . . . . . . . . . . . . . . . . . . . . . . . 128

\begin{tabular}{l|l|} 
D. Curriculum vitae & 131 \\
\hline
\end{tabular} 




\section{Introduction}

Note that this thesis is based on the publications by Krahn et al. ${ }^{[1]}$ and Lottmann et $a l .2]$.

\subsection{General introduction to dynamic nuclear polarization}

The theoretical background of nuclear magnetic resonance (NMR) spectroscopy is a broad and complex field and goes beyond the scope of this work. Therefore, only the basic principles which are most important under the aspect of dynamic nuclear polarization ( $\overline{\mathrm{DNP}}$ ) and thermal equilibrium polarization in the liquid state, are mentioned here. A more detailed description of the theory and methodology NMR spectroscopy can be found in the literature $\left.\underline{[}^{-5}-5\right]$.

NMR is based on the nuclear spin $\vec{I}$ as a quantum-mechanical phenomenon. The spin quantum number $I$ has integer or half-integer values. Some nuclei have no nuclear spin and therefore $I$ is equal to 0 . The nuclear spin is connected with a magnetic momentum $\vec{\mu}$. The ratio between $\vec{I}$ and $\vec{\mu}$ is constant and is described by the following equation:

$$
\vec{\mu}=\gamma \hbar \vec{I}
$$

where $\hbar$ is the reduced Planck's constant and $\gamma$ is the gyromagnetic ratio, which is a distinct property of each isotope with a nuclear spin. In a magnetic field $\vec{B}$, the magnetic moment of the nucleus leads to an alignment of the nuclear spin. It takes $2 I+1$ orientations relative to the magnetic field direction (z-axis). Each orientation has a different potential energy, which is given by:

$$
E_{m}=-\mu_{\mathrm{z}} B_{0}=-m_{\mathrm{z}} \gamma \hbar B_{0}
$$


with the magnetic momentum in field direction $\mu_{\mathrm{z}}$, the quantum number $m_{\mathrm{z}}$, the magnetic field in z-direction $B_{0}$ and the corresponding energy $E_{m} . m$ takes values of $I, I-1, \ldots,-I$. The difference between two $(m$ and $m+1)$ of these energy levels $\Delta E$ is constant:

$$
\Delta E_{(m+1)-m}=\gamma \hbar B_{0}
$$

Furthermore, the population of the energy states can be described by the Boltzmann distribution for thermal equilibrium:

$$
\frac{N_{i}}{\sum_{i} N_{i}}=\frac{g_{i} \mathrm{e}^{-E_{i} / k_{\mathrm{B}} T}}{\sum_{i} g_{i} \mathrm{e}^{-E_{i} / k_{\mathrm{B}} T}}
$$

with $N_{i}$ the number of nuclei in state i, the degeneracy $g_{\mathrm{i}}$ and $E_{\mathrm{i}}$ the energy of this state. The product of the Boltzmann constant $k_{\mathrm{B}}$ and the temperature $T$ gives the thermal energy of the nuclei.

The majority of the studied nuclei have an spin quantum number of $\frac{1}{2}$. This is due to the fact, that nuclei with higher quantum numbers possess electric quadrupole moments, which render NMR experiments more difficult with a low sensitivity. Spin $\frac{1}{2}$ nuclei have two distinct energy states in a magnetic field: the $\alpha$ state with $m_{\mathrm{z}}=\frac{1}{2}$ parallel to the magnetic field and the $\beta$ state with $m_{\mathrm{z}}=-\frac{1}{2}$ antiparallel, which has a higher energy than the former state. For these two states, equation 1.4 is used to determine the thermal equilibrium Boltzmann polarization ${ }^{[6]}$ with $g_{i}=1$ and equation 1.2 :

$$
P=\frac{N_{\alpha}-N_{\beta}}{N_{\alpha}+N_{\beta}}=\tanh \left(\frac{\hbar \gamma B_{0}}{2 k_{\mathrm{B}} T}\right)
$$

With the assumption $\hbar \gamma B_{0} \ll 2 k_{\mathrm{b}} T$, one can derive from equation 1.5 the following equation on the basis of the Taylor series:

$$
P \approx \frac{\hbar \gamma B_{0}}{2 k_{\mathrm{B}} T}
$$

By applying this equation to a proton containing sample in a field of $14.1 \mathrm{~T}(600 \mathrm{MHz}$ proton frequency) at a temperature of $20^{\circ} \mathrm{C}$ one gets a polarization of $5 \cdot 10^{-5}$. This corresponds to the detection of only one spin out of twenty thousand. This example underlines the statement that an increased polarization of the nuclei would be an 
important progress in NMR spectroscopy.

Over the last decades nuclear magnetic resonance (NMR) has been established as one of the most powerful and versatile analytical techniques on a molecular level. For example, it is used for identifying individual chemical components, determining the structure of complex macromolecules or studying dynamic processes and interactions.

All this was achieved despite the most significant drawback of NMR spectroscopy as an analytical method, namely sensitivity. For the measurements, the very weak magnetic moment of specific isotopes such as ${ }^{1} \mathrm{H},{ }^{13} \mathrm{C}$ or ${ }^{15} \mathrm{~N}$ and its properties in a magnetic field are exploited. This weakness renders the recording of NMR spectra time consuming or makes very high sample concentrations mandatory.

As recently summarized by Griffin and Prisner ${ }^{[7]}$, several technical and methodological developments have been implemented in order to reduce this disadvantage: These are namely Fourier transform spectroscopy ${ }^{[\underline{[}]}$, which allows to record all resonances in a spectrum at once instead of consecutively, superconductor magnets and cryoprobes ${ }^{[9]}$ as well as Hartmann-Hahn cross polarization ${ }^{[10,11]}$, J-coupling mediated ${ }^{[12]}$ transfer methods and ${ }^{1} \mathrm{H}$ detection of ${ }^{13} \mathrm{C}$ and ${ }^{15} \mathrm{~N}$ resonances ${ }^{[13]}$, respectively.

Another approach to increase the sensitivity is the transfer of polarization from an external source to the nuclei. One possible source is an unpaired electron which possesses a magnetic moment that is several magnitudes stronger than the magnetic moment of a nucleus and thereby its Boltzmann polarization, too. The polarization of the electron is 658 times larger than that of ${ }^{1} \mathrm{H}$ and 2617 times larger than that of ${ }^{13} \mathrm{C}$. For the first time ${ }^{[14]}$, the basic concept of this method has been developed and proposed by Overhauser in $1953^{[15]}$. By now it is known as Dynamic Nuclear Polarization (DNP).

Overhauser proposed that the polarization of the nuclei in a metal would increase when the transitions of the metal electrons in a magnetic field are saturated by irradiation with an electromagnetic wave. This increase is caused by the hyperfine interaction between the nuclei and the electrons. The saturated electron transitions represent a deviation from the thermal equilibrium polarization of the electrons and subsequently induce relaxation processes leading to an increased polarization of the nuclei. A more detailed description is given in section 1.3 . 
In the year 1953 Carver and Slichter ${ }^{[16]}$ proved Overhauser's proposition by enhancing the signal of $\mathrm{Li}^{7}$ nuclei up to 100-fold. Later on, I. Solomon published a detailed theoretical description ${ }^{[17]}$ for the Overhauser effect based on dipolar interactions between the intrinsic angular momenta (spins) of elementary particles. Solomon generalized the effect to a system of two spins in a magnetic field. These two spins can be alike or unlike and be nuclei and/or electrons. In his experiments, I. Solomon demonstrated that the Overhauser effect occurs between nuclei (Nuclear Overhauser Effect - NOE), too.

For the first time in 1956 a DNP experiment in liquid state was conducted by Carver and Slichter $\underline{[18]}$. They dissolved sodium in anhydrous liquid ammonia and saturated the transition of the dissolved free electrons. A more than -400 times increased proton polarization of ammonia was observed. Already, these first experiments clearly demonstrated the potential of DNP as a powerful method for NMR.

To this day, DNP has evolved into a complex and heterogeneous field of research. In literature $\underline{\underline{7}, 19,20]}$, a multitude of different approaches are described as will be seen in the following. These approaches aim for applications in liquid state and solid state NMR as well as in Magnetic Resonance Imaging (MRI). They can be categorized into four different groups ${ }^{\text {[7] }}$ : (I) magic angle spinning (MAS) DNP, (II) dissolution DNP, (III) high-field (hf) liquid state DNP and (IV) liquid state shuttle DNP.

MASIDNP gained a broader applicability in the 90's when high field ( $>5 \mathrm{~T}$ ) experiments had become feasible with the help of gyrotrons as a high power microwave


second important improvement in this field of research. Until today both aspects are still under current development and optimization. New gyrotrons reaching the terahertz regime ${ }^{[25]}$ are tested as well as more efficient polarizers ${ }^{[26]}$.

In the solid state, the electron polarization is transferred to the nuclei by three different effects ${ }^{[27-29]}$ : solid effect, cross effect and thermal mixing. The dominating mechanism as well as the efficiency of the polarization transfer depend on the temperature, the polarizing agent and the field of polarization. In MAS|DNP, most experiments are conducted at $100 \mathrm{~K}$ where signal enhancements factors of 235 for protons at $400 \mathrm{MHz}$ proton frequency and of 128 at $600 \mathrm{MHz}$, respectively ${ }^{[26]}$, are achieved. The proton polarization is subsequently used in MAS cross polarization experiments for carbon, nitrogen or silicon $\underline{[30,31]}$ detection. 
The second approach, dissolution DNP, is based on the same physical principles, but differs in its experimental procedure and application. The sample is polarized in the solid state, too, but at 1-4 K followed by dissolution with a hot solvent ${ }^{[32]}$. This dissolution step is irreversible and can be done only once per sample. Therefore, the main focus lies on the maximization of the initial polarization. In order to achieve this, heteronuclei are polarized, since they have more favorable relaxation properties in regard to the delay between polarization and detection of the sample. Here, slow relaxation is mandatory to avoid polarization losses.

Overall nuclear polarizations of up to $70 \%\left({ }^{13} \mathrm{C}\right)$ were reached for samples directly before the dissolution step ${ }^{[33]}$. So far, it has not been possible to gain the same polarization after the subsequent dissolution step, yet. However, a polarization of about $40 \%$ was measured in the dissolved sample. This corresponds to an enhancement factor of more than fifty thousand.

These hyperpolarized solutions are used for two different applications. One of these is the administration as contrast agent in MRI. In this context, the technique is used to monitor the metabolism of tumors by recording the carbon signals of previously hyperpolarized metabolites $\underline{[35,36]}$.

The second application is in liquid state NMR spectroscopy. The polarized solution is directly transferred into a NMR probe by rapid sample injection $\underline{\text { [37-39] }}$. With the high initial polarization and small flip angle excitation, it is possible to follow irreversible dynamic processes in the time scale of a few seconds such as chemical reactions ${ }^{[40]}$ or protein folding ${ }^{[41]}$. The disadvantage of this injection method in comparison to conventional liquid state NMR experiments is that signal averaging and proton detection are not possible or in a very limited way, respectively. Beside this, the dissolution process with hot solvents is not applicable to every sample and its chemical compounds or biomolecules.

An alternative, more direct approach to obtain DNP enhancements in liquid samples is the excitation of the unpaired electrons of the dissolved polarizer molecules by $m w$ irradiation in the liquid state. Under these conditions, the polarization is transferred via the Overhauser effect through space to the nuclei of the solution ${ }^{[6]}$. The efficiency of the transfer depends on the distance between electron and nucleus as well as the strength of the actual magnetic field (see 1.3.4).

Additionally, dielectric losses of the microwave irradiation represent a serious tech- 
nical complication in dipolar solvents as they cause severe heating of the sample. This heating effect is minimized by placing the sample within the resonator at the maximum of the magnetic field of the microwave and thereby at the minimum of electric field which causes the heating of the sample in the first place.

The sample diameter should only be a small fraction of the wavelength of the microwave to ensure a significant impact of this arrangement. Thus, the static magnetic field determines the wavelength and, consequently, the sample diameter which is linked to the sample volume. In other words, high static magnetic fields require small sample volumes. In comparison, these restrictions are absent in the previously described $\mathrm{DNP}$ approaches since the sample is in the solid state, where the dielectric losses are negligible.

In regard to these restrictions, two different approaches for liquid state DNP are realized: (III) hf liquid state DNP and (IV) liquid state shuttle DNP. The first approach has the disadvantages of very small sample volumes and an inefficient polarization transfer. In the second one, the field of polarization and detection are separated with the gain of moderate sample volumes and a more efficient polarization transfer. This separation makes a sample transfer between the fields mandatory which causes relaxation losses of the previously generated polarization.

In the literature, liquid state DNP set-ups at proton frequencies of $400 \mathrm{MHz}$ $(9.2 \mathrm{~T})$ and sample volumes of about 3 to $4 \mathrm{~nL}$ are described $\underline{\underline{42}-\underline{44}]}$. Enhancements of -13 at $45^{\circ} \mathrm{C}$ and -79 at $160{ }^{\circ} \mathrm{C}^{[45]}$ for water as well as -0.6 at $40{ }^{\circ} \mathrm{C}^{[46]}$ for methyl protons of pyruvate* were obtained.

As described before, the liquid state shuttle DNP approach utilizes a low magnetic field for a more efficient polarization transfer and bigger sample volumes. However, to obtain sufficient resolution for biological applications, a sample transfer to high magnetic fields is necessary. A first prototype $\underline{[47,48]}$ of a shuttle spectrometer with two dedicated magnets was built: one magnet operates at $0.34 \mathrm{~T}$ (9.7 GHz electron frequency) for the polarization of the nuclei (low-field - Ifl) and the other one at $14.09 \mathrm{~T}$ (600 MHz proton frequency) for high resolution NMR detection (high-field

\footnotetext{
${ }^{*}$ Note that the enhancement factors are back calculated to fit the definition (equation 1.22) given below, which is based on the publication by K.H. Hausser and D. Stehlik $[6]$. The factor given in these publications ${ }^{[42-46]}$ are defined differently and would correspond to the definition of polarization in this work.
} 
- hf). The centers of these two magnets are $1.525 \mathrm{~m}$ apart. A pneumatic shuttle system transfers a sample of $10 \mu \mathrm{L}$ within 100 to $200 \mathrm{~ms}$ (start of transfer to signal acquisition). With the help this setup, it was proven, that it is possible to polarize protons of water at a low magnetic field and transfer the sample to the high magnetic field with a resulting signal enhancement of -3.5.

However, the sample passes through a magnetic field minimum of about $4.5 \mathrm{mT}$ during the transfer from the $\lfloor f$ to the hf position, where the relaxation times of the nuclei become very short. With increasing molecular size, this effect becomes more severe because larger molecules have even shorter relaxation times. Therefore, it was impossible to preserve the dynamic nuclear polarization, which was generated in the [f], for dissolved molecules.

Shortly before the beginning of this $\mathrm{PhD}$ project, a new setup was designed featuring a two center magnet ${ }^{[1]}$ with a continuously increasing magnetic field strength from the If $(0.34 \mathrm{~T})$ to the $\mathrm{hf}(14.1 \mathrm{~T})$ position. The If field plateau is accomplished by an additional ferroshim system in the stray field within the bore of the hf cryo magnet. Furthermore, a new DNP probe was made to fit in this novel shim system. This probe operated in the $\mathrm{TM}_{110}$ microwave mode. At this point it is referred to chapter 2 for more details due to numerous similarities of the principle setup design to the actual setup used in this thesis.

With this new setup, it was possible to polarize dissolved molecules in water and preserve most of the previously generated polarization for measurements in the hf position despite the relaxation losses during the transfer of the sample. This was demonstrated for D-glucose with an enhancement of up to -2.8 at $60{ }^{\circ} \mathrm{C}$. These findings are the basis for the ensuing efforts of this thesis.

In summary, the liquid state shuttle DNP spectrometer set-up aims to acquire structural and/or dynamic information of biological samples (with water as mandatory solvent) in the liquid state (different from MAS DNP) with rapid and repetitive signal acquisition. This means, that this set-up enables the implementation of conventional, multidimensional NMR experiments and features sample preservation which becomes essential, when only a small amount of material is available (different from dissolution (DNP). In addition, a high efficiency is obtained for the polarization transfer due to the more favorable lower magnetic fields (different from hf liquid state DNP). 


\subsection{Aims and outline}

Krahn et $a l .{ }^{[1]}$ presented a first prototype of a shuttle DNP spectrometer with a two-center magnet. They polarized dissolved (non-solvent) molecules for the first time in the liquid state and detected them at high fields.

The first aim of this thesis is to continue the efforts mentioned above and increase the reproducibility and stability of the shuttle $\mathrm{DNP}$ spectrometer with a two-center magnet in cooperation with Bruker Biospin (Rheinstetten, Germany), in order to enable measurements of several hours and several thousand scans, such as multidimensional correlation experiments. For this purpose, several major improvements of the shuttle DNP spectrometer are implemented and tested, such as a generally overhauled DNP probe, active cooling of the If section of the magnet and a newly designed shuttle container. In this process, a more complete assessment of the signal enhancement is established in regard to the obtained signal-to-noise ratio (SNR) per measurement time. For this purpose, important factors are taken into account, such as line broadening or changed acquisition rates due to the paramagnetic polarizer in the sample solution.

The second aim is to study the polarization transfer between the nuclear spins and the electron spins. Based on the coupling factor, it is investigated, how the accessibility of the target nuclei by the polarizer molecule influences the polarization transfer and how it can be theoretically described. Furthermore, heteronuclear spin systems with more than one nuclear spin are taken into account.

As a concluding step, the third aim is to measure heteronuclear correlation spectra of a protein with $\mathrm{DNP}$ based on the previously obtained results. 


\subsection{Theory of liquid state shuttle DNP}

\subsubsection{Basics of shuttle Overhauser DNP}

As described before (1.1), the idea of DNP is to increase the low Boltzmann polarization of nuclei via interacting electrons in a magnetic field. I. Solomon theoretically described the transfer processes in a generalized system of two dipolar coupled spins with the value $\frac{1}{2}[17]$. His approach is based on the Hamiltonian of two spins $\vec{S}$ (unpaired electron) and $\vec{I}$ (nucleus) interacting in a magnetic field $\vec{B}$ :

$$
\mathscr{H}=\mathscr{H}_{\mathrm{M}}-\hbar \gamma_{I}(\vec{B} \cdot \vec{I})-\hbar \gamma_{S}(\vec{B} \cdot \vec{S})+\mathscr{H}_{I S}
$$

$\mathscr{H}_{\mathrm{M}}$ is the Hamiltonian of motion of the two spins followed by the respective Zeeman energies. $\mathscr{H}_{I S}$ is the spin-spin interaction term, which is considered as a fast fluctuating perturbation (as for example in gases and liquids). For a pure dipole-dipole interaction the Hamiltonian is described as follows:

$$
\mathscr{H}_{I S}=-\left(\frac{\hbar^{2} \gamma_{I} \gamma_{S}}{r^{3}}\right)[3(\vec{I} \cdot \vec{r})(\vec{S} \cdot \vec{r})-\vec{I} \cdot \vec{S}]
$$

$\vec{r}$ is the vector between the two dipoles and $r$ is the distance. Based on equation 1.8 Solomon ${ }^{[17]}$ derived the transition probabilities between the four energy states of

Figure 1.1.: The four energy states of a two spin system in a magnetic field. $\vec{S}$ represents the electron spin and $\vec{I}$ a nuclear spin with the value $\frac{1}{2}$. Both spins are dipolar coupled. $w_{i}$ is the transition probability and $\Delta E$ the energy difference between the indicated states with the gyromagnetic ratio $\gamma$, the reduced Planck constant $\hbar$ and the magnetic field $B_{0}$ along the z-axis

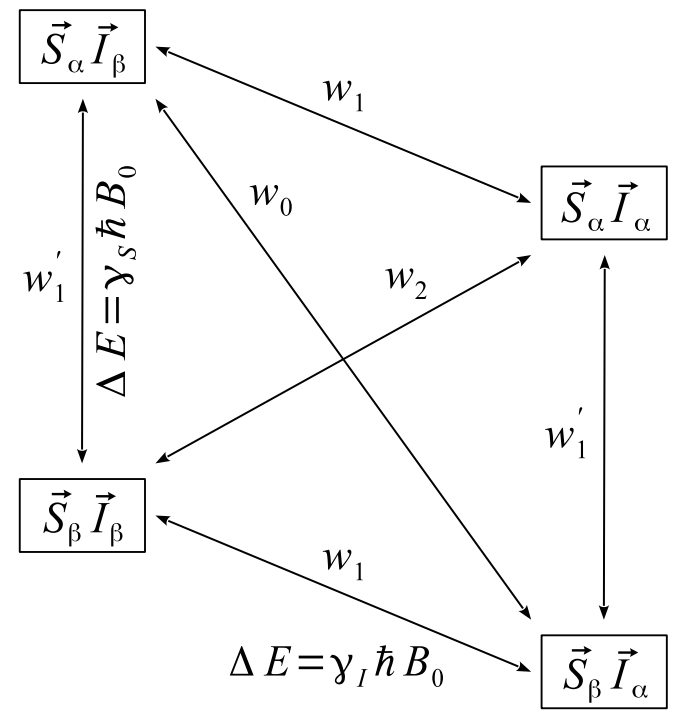


the two interacting spins (see fig. 1.1) and the change of the macroscopic magnetic moments $I_{z}$ and $S_{z}$ :

$$
\begin{aligned}
& \frac{d I_{\mathrm{z}}}{d t}=-\left(w_{0}+2 w_{1}+w_{2}\right)\left(I_{\mathrm{z}}-I_{0}\right)-\left(w_{2}-w_{0}\right)\left(S_{\mathrm{z}}-S_{0}\right) \\
& \frac{d S_{\mathrm{z}}}{d t}=-\left(w_{2}-w_{0}\right)\left(I_{\mathrm{z}}-I_{0}\right)-\left(w_{0}+2 w_{1}^{\prime}+w_{2}\right)\left(S_{\mathrm{z}}-S_{0}\right)
\end{aligned}
$$

$I_{0}$ and $S_{0}$ are the thermal equilibrium values of the macroscopic magnetic moments. For a general and more realistic description, one must consider additional transitions beside the dipolar spin-spin interaction. They can occur due to further perturbations of the spin states in a sample. All additional externally induced transitions are summarized in $w^{0}$ as an overall transition probability of the two spins $\underline{\underline{6}}$.

$$
\begin{array}{r}
\frac{d I_{\mathrm{z}}}{d t}=-\left(w_{0}+2 w_{1}+w_{2}+w^{0}\right)\left(I_{\mathrm{z}}-I_{0}\right)-\left(w_{2}-w_{0}\right)\left(S_{\mathrm{z}}-S_{0}\right) \\
\frac{d S_{\mathrm{z}}}{d t}=-\left(w_{2}-w_{0}\right)\left(I_{z}-I_{0}\right)-\left(w_{0}+2 w_{1}^{\prime}+w_{2}+w^{\prime 0}\right)\left(S_{\mathrm{z}}-S_{0}\right)
\end{array}
$$

Under steady state conditions with $\frac{d I_{z}}{d t}=0$, equation 1.11 is rearranged to:

$$
I_{z}=I_{0}+\left(\frac{w_{2}-w_{0}}{w_{0}+2 w_{1}+w_{2}}\right)\left(\frac{w_{0}+2 w_{1}+w_{2}}{w_{0}+2 w_{1}+w_{2}+w^{0}}\right)\left(S_{0}-S_{z}\right)
$$

and simplified by introducing the auto-relaxation rate $\rho$ and the cross-relaxation rate $\sigma$ with:

$$
\begin{aligned}
& \rho=w_{0}+2 w_{1}+w_{2} \\
& \sigma=w_{2}-w_{0}
\end{aligned}
$$

to:

$$
I_{z}=I_{0}+\left(\frac{\sigma}{\rho}\right)\left(\frac{\rho}{\rho+w^{0}}\right)\left(S_{0}-S_{z}\right)
$$

A commonly measured quantity in DNP experiments is the signal enhancement 
which is defined as:

$$
\varepsilon=\frac{I_{z}}{I_{0}}
$$

By inserting equation 1.16 in 1.17 and multiplying by $S_{0} / S_{0}$ one gets:

$$
\varepsilon=1+\left(\frac{\sigma}{\rho}\right)\left(\frac{\rho}{\rho+w^{0}}\right)\left(\frac{S_{0}-S_{z}}{S_{0}}\right)\left(\frac{S_{0}}{I_{0}}\right)
$$

For this equation, A. Abragam ${ }^{[49]}$ introduced the following definitions for the coupling factor $\xi$, the leakage factor $f$ and saturation factor $s$ :

$$
\begin{aligned}
& \xi=\frac{\sigma}{\rho} \\
& f=\frac{\rho}{\rho+w^{0}} \\
& s=\frac{S_{0}-S_{z}}{S_{0}}
\end{aligned}
$$

All three parameters reflect an important aspect of the DNP process: excitation $(s)$, transfer $(\xi)$ and losses $(f)$. These factors are described in more detail in the following subsections (1.3.3 and 1.3.4). However, the quotient of the two macroscopic magnetic moments $S_{0} / I_{0}$ can be replaced by the quotient of the two corresponding gyromagnetic ratios $\gamma_{S} / \gamma_{I}$, which gives the final equation for the DNP enhancement in the liquid state at a specific field:

$$
\varepsilon=1+\xi f s \frac{\gamma_{S}}{\gamma_{I}}
$$

Under ideal conditions, the dipolar coupling factor $\xi$ becomes 0.5 . No polarization is lost through competing relaxation pathways with $f=1$ and the electron transition is completely saturated with $s=1$. With the gyromagnetic ratios of a proton $\left(\gamma_{\mathrm{I}, \mathrm{H}}=2.675222005 \cdot 10^{8} 1 / \mathrm{sT}\right)$ or a carbon $\left(\gamma_{\mathrm{I}, \mathrm{C}}=0.6728286 \cdot 10^{8} 1 / \mathrm{sT}\right)$ and an electron $\left(\gamma_{\mathrm{S}, \mathrm{H}}=-1.76085970839 \cdot 10^{11} 1 / \mathrm{sT}\right)$, the theoretical maximum enhancement $\varepsilon_{\text {th }}$ is -328 for proton or -1308 for carbon without sample transfer. 


\subsubsection{Shuttle DNP}

Since the shuttle DNP approach involves two fields, the equation 1.17 for the DNP enhancement must be adapted to this circumstance. In general, the achievable signal enhancement $\varepsilon_{\text {hf }}$ at the high field position is of interest, where the signal is acquired. The generated dynamic nuclear polarization $I_{z, \text { lf }}$ in the low field position is diminished to $I_{z, \text { lf }}^{\prime}$ in the high field position due to relaxation during the sample transfer between these two fields. The reduced polarization $I_{z, l f}^{\prime}$ is referenced to the thermal equilibrium polarization of the high field position $I_{0, \mathrm{hf}}$ to obtain $\varepsilon_{\mathrm{hf}}$ :

$$
\varepsilon_{\mathrm{hf}}=\frac{I_{\mathrm{z}, \mathrm{lf}}^{\prime}}{I_{0, \mathrm{hf}}}
$$

The relaxation losses $\lambda$ during the transfer are defined as:

$$
\lambda=1-\frac{I_{\mathrm{z}, \mathrm{lf}}^{\prime}}{I_{\mathrm{z}, \mathrm{lf}}}
$$

This gives together with equation 1.17 and 1.22 ;

$$
\varepsilon_{\mathrm{hf}}=\frac{I_{0, \mathrm{lf}}}{I_{0, \mathrm{hf}}}\left(1+\xi f s \frac{\gamma_{S}}{\gamma_{I}}\right)(1-\lambda)
$$

The ratio of $I_{0, \text { lf }}$ and $I_{0, \mathrm{hf}}$ is proportional to the field strength of the low field $B_{0, \mathrm{lf}}$ (polarization) and the high field $B_{0, \mathrm{hf}}$ (detection), which finally gives:

$$
\varepsilon_{\mathrm{hf}}=\frac{B_{0, \mathrm{lf}}}{B_{0, \mathrm{hf}}}\left(1+\xi f s \frac{\gamma_{S}}{\gamma_{I}}\right)(1-\lambda)
$$

The theoretical maximum enhancement for protons and carbons in a shuttle DNP spectrometer with two magnetic fields at $0.34 \mathrm{~T}$ and $14.1 \mathrm{~T}$ is according to subsection $1.3 .1 \varepsilon_{\mathrm{H}, \mathrm{hf}, \mathrm{th}}=-328 \cdot \frac{0.34}{14.1}=-7.9$ and $\varepsilon_{\mathrm{C}, \mathrm{hf}, \mathrm{th}}=-1308 \cdot \frac{0.34}{14.1}=-31.6$, respectively, with the assumption, that no relaxation losses occur $(\lambda=0)$ during the transfer. 


\subsubsection{Leakage and saturation factor}

The leakage factor reflects how much of the transferred polarization is lost through relaxation pathways of the nuclear spin $\vec{I}$, which do not involve the electron spin $\vec{S}$. This becomes more clear by expressing equation 1.20 in a different manner ${ }^{[6,50]}$ :

$$
f=\frac{\rho}{\rho+w^{0}}=\frac{R_{\text {para }}}{R_{1, \text { para }}+R_{1, \text { dia }}}=\frac{R_{1, \text { rad }}-R_{1, \text { no_rad }}}{R_{1, \text { rad }}}
$$

$R_{1, \text { para }}$ is the sole paramagnetic relaxation rate and $R_{1, \text { dia }}$ the diamagnetic relaxation rate. This relation can be used to experimentally determine $f$ measuring the relaxation rate of the nucleus in the presence $R_{1, \text { rad }}$ and the absence $R_{1 \text {,no_rad }}$ of the spin $\vec{S}$ and the radical, respectively. Furthermore it becomes obvious, that nuclei with high diamagnetic relaxation rates tend to smaller leakage factors compared to nuclei with lower rates. The consequence is a smaller enhancement.

The saturation factor is the normalized deviation of the magnetization of the electron spin $\vec{S}$ from thermal equilibrium (equation 1.21). By irradiating the hyperfine line of $\vec{S}$ with a electromagnetic wave, the spin state population difference is equalized and therefore $S_{\mathrm{z}}$ becomes zero. In this case, the Bloch equations ${ }^{[51]}$ for the rotating frame result in the following equation with the steady state condition $\frac{d S_{\mathrm{z}}}{d t}=0$ and on resonance irradiation:

$$
S_{\mathrm{z}}=S_{0} \frac{1}{1+g_{\mathrm{e}}^{2} \mu_{\mathrm{B}}^{2} \tau_{1, S} \tau_{2, S} B_{1}^{2}}
$$

$\tau_{1, S}$ and $\tau_{2, S}$ are the the longitudinal and transverse relaxation times of $\vec{S}$, respectively. $B_{1}$ is the magnetic field strength of the applied electromagnetic wave. By combining equation 1.21 and 1.28, one gets for $s$ the following expression ${ }^{[52]}$ :

$$
s=1-\frac{1}{1+g_{\mathrm{e}}^{2} \mu_{\mathrm{B}}^{2} \tau_{1, S} \tau_{2, S} B_{1}^{2}}
$$

This result is only valid if all electron transitions and therefore hyperfine lines are pumped at the same time. For the specific case of exciting one of multiple lines, additional interactions must be taken into account.

The most important effect in the context of shuttle DNP is Heisenberg exchange. 
Collisions between the radical bearing molecules lead to an exchange of electron spins between the hyperfine lines $[\underline{53,54]}$. Thus, electron population differences of different nuclear spin configurations begin to equalize depending on the collision rate and exchange rate, respectively.

For an occurring exchange, the two electron spins as well as the nuclear environment of the colliding molecules must be different to result in an observable effect. When the molecules separate again, the probability is $\frac{1}{2}$ that the states of the two electrons switched between the two unaffected nuclear environments. An extended theoretical treatment of the saturation behavior of electrons with multiple hyperfine lines in solution was done by Freed $[\underline{55}, 56]$. The only constraint of his approach is the limitation to low radical concentrations since he excluded collisions of more than two radical molecules at the same time.

Türke et al. [57,58] applied Freed's approach to nitroxide radicals with ${ }^{15} \mathrm{~N}$ label. The spin of ${ }^{15} \mathrm{~N}$ is $I=\frac{1}{2}$ which gives two hyperfine lines. In their approach, only one hyperfine line is effected by the $\mathrm{mw}$ irradiation and saturated. With these conditions, the corresponding effective saturation factor $s_{\text {eff }}$ for the overall saturation of both lines was derived:

$$
s_{\mathrm{eff}}=\left(\frac{2\left(2 w_{1 \mathrm{e}}+w_{1 \mathrm{n}}\right)+\omega_{\mathrm{ex}}}{2\left(w_{1 \mathrm{e}}+w_{1 \mathrm{n}}\right)+\omega_{\mathrm{ex}}}+\frac{2}{g_{\mathrm{e}}^{2} \mu_{\mathrm{B}}^{2} \tau_{1, S} \tau_{2, S} B_{1}^{2}}\right)^{-1}
$$

$w_{1 \mathrm{e}}$ and $w_{1 \mathrm{n}}$ are the intramolecular equivalents of the intermolecular single quantum transition probabilities $w_{1}^{\prime}$ and $w_{1}$ for two spins (see figure 1.1). $\omega_{\text {ex }}$ is the exchange rate between the two hyperfine lines along with $\tau_{1, S}$ and $\tau_{2, S}$ as the longitudinal and transverse relaxation time of the unpaired electron, respectively. $B_{1}$ is the magnetic field of the electromagnetic wave which is used for the saturation. It immediately is evident from equation 1.30 that the exchange rate has an important impact on the effective saturation factor and, therefore, on the enhancement. 


\subsubsection{Coupling factor}

The coupling factor reflects the efficiency of the dipolar polarization transfer. The higher the coupling factor the more polarization is transferred from the unpaired electrons to the nuclei. As illustrated by equation 1.19, the coupling factor is the ratio between the cross-relaxation rate and the auto-relaxation rate of the nucleus and the electron. These two rates are based on the transition probabilities in a two spin system (figure 1.1) with zero quantum $w_{0}$, single quantum $w_{1}$ or $w_{1}^{\prime}$ and double quantum $w_{2}$ transitions. These probabilities are proportional to the spectral density function $J(\omega, \tau)$ of each transition $\underline{[17,50,59,60]}$ :

$$
\begin{aligned}
& w_{0}=k J\left(\omega_{I}-\omega_{S}, \tau\right) \\
& w_{1}=\frac{3}{2} k J\left(\omega_{I}, \tau\right) \\
& w_{2}=6 k J\left(\omega_{I}+\omega_{S}, \tau\right)
\end{aligned}
$$

$\omega$ is the Lamor frequency of the respective spins, $\tau$ the correlation time between the spins and $k$ a proportionality factor.

An alternative and the transition probabilities containing expression of the coupling factor is obtained by inserting equations 1.14 and 1.15 in 1.19 .

$$
\xi=\frac{w_{2}-w_{0}}{w_{0}+2 w_{1}+w_{2}}=\frac{6 k J\left(\omega_{I}+\omega_{S}, \tau\right)-k J\left(\omega_{I}-\omega_{S}, \tau\right)}{6 k J\left(\omega_{I}+\omega_{S}, \tau\right)+3 k J\left(\omega_{I}, \tau\right)+k J\left(\omega_{I}-\omega_{S}, \tau\right)}
$$

The coupling factor reaches its maximum with 0.5 for pure dipolar interactions under ideal conditions with $J\left(\omega_{I}+\omega_{S}, \tau\right) \approx J\left(\omega_{I}, \tau\right) \approx J\left(\omega_{I}-\omega_{S}, \tau\right)$. Under these conditions, no scalar coupling occurs.

In the literature ${ }^{[17,50,60,61]}$, different models were applied to calculate the proportionality factors and the spectral density functions for these transitions. In NMR, the most prominent model describes the dipolar spin-spin interaction between spins within a single molecule ${ }^{[17]}$. The strength of this intramolecular interaction is determined by the molecular tumbling or more precisely the rotational diffusion. The spectral density function $J_{\text {tumb }}\left(\omega, \tau_{\mathrm{c}, \mathrm{I}}\right)$ of this interaction depends on the rotational 
correlation time $\tau_{\mathrm{c}, \mathrm{I}}$ of the specific molecule and the Lamor frequency $\omega$ :

$$
J_{\text {tumb }}\left(\omega, \tau_{\mathrm{c}, \mathrm{I}}\right)=\frac{\tau_{\mathrm{c}, \mathrm{I}}}{1+\left(\omega \tau_{\mathrm{c}, \mathrm{I}}\right)^{2}}
$$

The proportionality factor of the mentioned transition probabilities in a two spin system is given by :

$$
k_{\text {tumb }}=\frac{1}{10}\left(\frac{\mu_{0}}{4 \pi}\right)^{2}\left(\frac{\gamma_{\mathrm{I}, 1}^{2} \gamma_{\mathrm{I}, 2}^{2} \hbar^{2}}{r_{12}^{6}}\right)
$$

$\mu_{0}$ is the vacuum permeability, $\gamma_{\mathrm{I}, 1}$ and $\gamma_{\mathrm{I}, 2}$ the gyromagnetic ratios of the coupled nuclei as well as $r_{12}$ the distance between the two nuclear spins $\vec{I}_{\mathrm{I}, 1}$ and $\vec{I}_{\mathrm{I}, 2}$.

This model of single molecular tumbling does not directly apply to the intermolecular interaction of the DNP experiment since the electron spin $\vec{S}$ and the nuclear spin $\vec{I}$ are located in two separate molecules which move independently of each other. This relative movement of the two spins is accounted for by a model based on translational diffusion and/or a for this context adapted model of the rotational diffusion.

Bennet et al. [61] introduced the term "Outer Sphere Relaxation" (outer sphere model) for the intermolecular dipolar spin-spin interaction based on translational diffusion. A frequently ${ }^{[50,60,61]}$ used mathematical approach for this interaction is the force-free model ${ }^{[62,63]}$ which accounts for the finite size of the diffusing molecules. The spectral density function $J_{t}\left(\omega, \tau_{t}\right)$ depending on the Lamor frequency $\omega$ and the translational diffusional correlation time $\tau_{\mathrm{t}} \underline{[50]}$ is defined as

$$
J_{t}\left(\omega, \tau_{t}\right)=\frac{1+\frac{5}{8} z+\frac{1}{8} z^{2}}{1+z+\frac{1}{2} z^{2}+\frac{1}{6} z^{3}+\frac{4}{81} z^{4}+\frac{1}{81} z^{5}+\frac{1}{648} z^{6}}
$$

with

$$
z=\sqrt{2 \omega \tau_{\mathrm{t}}}
$$

and the proportionality factor $k_{\mathrm{t}}$ :

$$
k_{t}=\frac{32 \pi}{405}\left(\frac{\mu_{0}}{4 \pi}\right)^{2} \frac{N_{\mathrm{A}}[S] \gamma_{I}^{2} g_{\mathrm{e}}^{2} \mu_{\mathrm{B}}^{2} S(S+1)}{d\left(D_{S}+D_{I}\right)}
$$

$N_{\mathrm{A}}$ is the Avogadro constant and $d$ the distance of closest approach between the 
two spins. Furthermore $D_{S}$ and $D_{I}$ are the diffusion constants of the spin bearing molecules. These diffusion constants also contribute to the translational diffusional correlation time $\tau_{\mathrm{t}}$ along with the distance of closest approach $d$ between the two spins:

$$
\tau_{\mathrm{t}}=\frac{d^{2}}{D_{S}+D_{I}}
$$

In addition, it is possible to extend the outer sphere model by the "Inner Sphere Relaxation", how the intermolcular interaction based on rotational diffusion was called by Bennet et al. $\underline{61]}$. The combination of inner and outer sphere relaxation is called (in this work) the combined model.

For the occurrence of inner sphere relaxation alongside outer sphere relaxation, the assumption is made, that both the electron and the nuclear spin bearing molecules stay for a short period of time $t_{\mathrm{r}}$ in close vicinity to each other (in the inner sphere), for example due to the formation of a weak complex ${ }^{[64,65]}$, hydrogen bonds ${ }^{[60]}$, temporary van-der-Waals interactions, etc.

Beyond this superficial and general description, no further more detailed definitions are made in regard to the inner sphere. Based on these assumptions, the intermolecular rotational correlation time $\tau_{\mathrm{r}}$ is defined as $\underline{\underline{[50]}}$ :

$$
\tau_{\mathrm{r}}=\left(\tau_{\mathrm{c}, \mathrm{S}}^{-1}+\tau_{1, S}^{-1}+\tau_{\mathrm{M}}^{-1}\right)^{-1}
$$

Here, $\tau_{\mathrm{c}, \mathrm{S}}$ is the rotational correlation time of the electron spin bearing molecule, $\tau_{1, S}$ the longitudinal relaxation time of the electron and $\tau_{\mathrm{M}}$ is the life time of the interacting nucleus in close vicinity to the electron spin. The Lorentzian spectral density function $J_{\mathrm{r}}\left(\omega, \tau_{\mathrm{r}}\right)$ for the inner sphere relaxation is given by:

$$
J_{\mathrm{r}}\left(\omega, \tau_{\mathrm{r}}\right)=\frac{\tau_{\mathrm{r}}}{1+\left(\omega \tau_{\mathrm{r}}\right)^{2}}
$$

which is identical to intramolecular case (equation 1.35). However, the proportion-

\footnotetext{
${ }^{\dagger}$ In the literature, the correlation time of the rotational diffusion interaction between two molecules is often only called rotational correlation time $\tau_{\mathrm{c}}$. This nomenclature may lead to confusion since $\tau_{\mathrm{c}}$ is used for the rotational correlation time of a single molecule, too. Therefore $\tau_{\mathrm{r}}$ is introduced in this work for a clear distinction.
} 
ality factor is different

$$
k_{\mathrm{r}}=\frac{2}{15} \frac{n[S]}{[I]}\left(\frac{\mu_{0}}{4 \pi}\right)^{2}\left(\frac{\gamma_{I}^{2} g_{\mathrm{e}}^{2} \mu_{\mathrm{B}}^{2} S(S+1)}{r^{6}}\right)
$$

$\mu_{0}$ is the vacuum permeability, $\gamma_{I}$ the gyromagnetic ratio of the nucleus, $g_{\mathrm{e}}$ the Landé g-factor of the electron, $\mu_{\mathrm{B}}$ the Bohr magneton and $r$ the distance between $\vec{I}$ and $\vec{S}$. $[I]$ and $[S]$ are the concentrations of the two spins. $n$ represents the temporal average number of spins $\vec{I}$ in the coordination sphere of $\vec{S}$. For a significant contribution of the inner sphere relaxation to the coupling factor, $\tau_{\mathrm{r}}$ should be at least of similar magnitude as the translational diffusion correlation time $\tau_{\mathrm{t}}$.

Since the transition probabilities of inner and outer sphere relaxation add up ${ }^{[\underline{6} .50]}$, the coupling factor of the combined model is given by:

$$
\xi=\frac{\left(w_{2, \mathrm{r}}+w_{2, \mathrm{t}}\right)-\left(w_{0, \mathrm{r}}+w_{0, \mathrm{t}}\right)}{\left(w_{0, \mathrm{r}}+w_{0, \mathrm{t}}+2 w_{1, \mathrm{r}}+2 w_{1, \mathrm{t}}+w_{2, \mathrm{r}}+w_{2, \mathrm{t}}\right)}
$$

where the transition probabilities with the index $\mathrm{r}$ represent the contribution of rotational diffusion and with the index the contribution of translational diffusion, respectively.

Both approaches, the translational diffusion model and the combined model were developed based on Nuclear Magnetic Relaxation Dispersion (NMRD) measurements $\underline{[60,62,63]}$ in order to describe the relaxation properties of solvents $\underline{[59,62,63,65]}$ in the presence of dissolved paramagnetic substances at different fields and temperatures. However, there is no clear evidence in literature, if the pure translational diffusion model or the combined model should be applied to the DNP experiments.

NMRD measurements by Polnaszek et al. ${ }^{[60]}$ and Bennati et al. ${ }^{[50]}$ showed for the system of nitroxide radicals dissolved in water, that the dependency of the paramagnetic relaxation rate of the water protons on the Larmor frequency is described more accurately by using the combined model of the inner and outer sphere relaxation. Nevertheless Polnaszek et al. $\underline{[60]}$ were not able to obtain reasonable values of $\tau_{\mathrm{r}}$ for a nitroxide spin labeled protein $\underline{[60]}$ and neglected the inner sphere relaxation for this system. The nitroxide spin labeled protein confirms that the contribution of the rotational diffusion strongly depends on the reorientation time of nitroxide radical. In contrast, Bennett et al. $\underline{\underline{61]}}$ reported evidence that the rotational diffusion 




Figure 1.2.: Dependency of the coupling factor on the proton Lamor frequency for the inner sphere model (dashed line), the outer sphere model (dotted line) and the combined model (full line). The calculations were conducted for water with $25 \mathrm{mM}$ TEMPONE- $\mathrm{d}_{16}{ }^{15} \mathrm{~N}$ at $298 \mathrm{~K}$. They are based on measurements and experimental values of Bennati et al. ${ }^{[50]}$ with $\tau_{\mathrm{r}}=20 \mathrm{ps}, D_{S}+D_{I}=2.87 \cdot 10^{-9} \mathrm{~m}^{2} \mathrm{~s}^{-1}, d=272 \mathrm{pm}$, $r=296 \mathrm{pm}$ and $n=2$.

is negligible for nitroxide radicals in water, but becomes relevant for macromolecular complexes.

Furthermore, $n$, the temporal average number of nuclear spins $\vec{I}$ in the coordination (or inner) sphere of the electron spin $\vec{S}$ is difficult to determine for nuclear spins $\vec{I}$, which are located on dissolved molecules. Until now the combined model has been only applied to solvents. Numbers of $n=2$ and $n=12$ are reported in literature for water protons ${ }^{[50]}$ near the unpaired electron of a nitroxide radical $t$ and for water protons ${ }^{[64]}$ in the coordination sphere of a manganese(II) ion, respectively.

For low concentrated $(<1 \mathrm{M})$ dissolved target molecules, one must assume, that these are not continuously present in the coordination sphere of the spin $\vec{S}$, which is in contrast to the always present solvent molecules. This circumstance would lead to a temporal average number of nuclear spins $n$ smaller than 1 . Altogether, it is necessary to asses the models based on the measured DNP data later on.

The differences between the two models are illustrated by the dependency of the coupling factor on the proton Lamor frequency in figure 1.2. The field profile of the coupling factor of water with $25 \mathrm{mM}$ TEMPONE- $\mathrm{T}_{16},{ }^{15} \mathrm{~N}$ at $298 \mathrm{~K}$ was calculated for

\footnotetext{
${ }^{\ddagger} n$ was back calculated from the fitted data.
} 
pure outer sphere relaxation (dotted line), pure inner sphere relaxation (dashed line) and the combination of them (full line). The calculations are based on measurements and experimental values of Bennati et al. $\underline{[50]}$.

Figure 1.2 evidently illustrates how the coupling factor increases towards lower Lamor frequencies and therefore implies, how the enhancement factors increase with decreasing magnetic field strength. As mentioned above, these higher enhancements are one of the advantages of the shuttle DNP approach with low field polarization. 


\section{The DNP shuttle spectrometer with a two center magnet}

\subsection{Overview}

The DNP shuttle spectrometer with a two center magnet consists of three essential parts: The If section, where the sample is polarized, the pneumatic shuttle system, which transfers the sample between the two field plateaus, and the hf section, where the NMR signal is acquired with high resolution (figure 2.1).

For the low field part, an extra ferroshim system was installed inside the magnet bore $(54 \mathrm{~mm})$ of a NMR $14.1 \mathrm{~T}$ shielded cryomagnet ( ${ }^{1} \mathrm{H} 600 \mathrm{MHz}$ "UltraShield", Bruker). It generates a second homogeneous field plateau of $0.34 \mathrm{~T}$, which is located $47 \mathrm{~cm}$ above the center of the cryomagnet. A DNP probe is mounted from the top, placing the EPR cavity in the center of the ferroshim system. The cavity is tuned to a frequency of $9.6 \mathrm{GHz}$, which corresponds to the Lamor frequency of an electron at 0.34 T. A Bruker E-Scan EPR spectrometer with an additional $20 \mathrm{~W}$ amplifier (Varian MED VZX6981K1) generates the microwave for the DNP experiments and also drives additional sweep and modulation coils inside the ferroshim system and in the cavity, respectively.

A dedicated shuttle controller built by Bruker transfers the sample pneumatically from the DNP probe to the NMR probe in the hf position within $65 \mathrm{~ms}$. For this purpose, a special sample container was designed to withstand the strain imposed by the rapid transfer. It can hold up to $5 \mu \mathrm{L}$ of sample solution. Both the shuttle controller and the gate of the microwave amplifier are triggered using a $600 \mathrm{MHz}$ Bruker Avance III console.

A modified high resolution NMR probe (dual ${ }^{1} \mathrm{H} /{ }^{13} \mathrm{C}$ with ${ }^{2} \mathrm{H}$ lock channel) is 
used for signal detection. High reproducibility and stability make signal averaging and fast acquisition rates possible.

In the next section, the spectrometer and its components will be described in more detail.

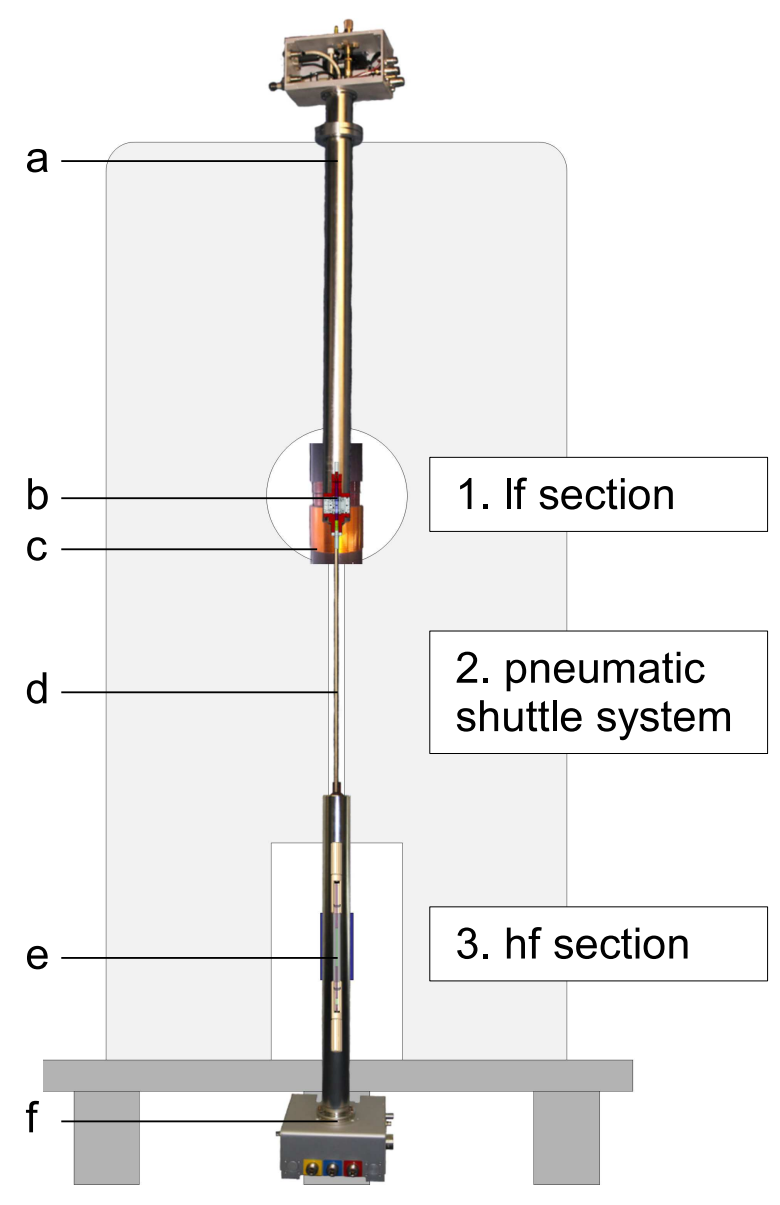

Figure 2.1.: The schematic overview illustrates the DNP shuttle spectrometer with its three essential parts. The DNP probe (a) with the DNP cavity (b) centered in the ferroshim system (c) is mounted from the top of the magnet. A transfer tube (d) connects the cavity to the NMR probe (f), which is mounted from the bottom of the magnet. The shuttle container (e) is transferred between the two probes by a pneumatic shuttle system 


\subsection{Low field part}

\subsubsection{Low field plateau}

As described in section 1.1, it is crucial to minimize polarization losses between the generation of the polarization and the detection of the NMR signal. This could be achieved in two ways: (i) by a very fast sample transfer and (ii) by ensuring long relaxation times of the polarized nuclei during the transfer. Both aspects are addressed by the implementation of the ferroshim system.

The ferroshim system is an additional shim system, which is used to adjust a certain section of the stray field of the hf cryomagnet. It is composed of a ferromagnetic metal cylinder which was optimized for compensating the specific stray field gradient around $0.34 \mathrm{~T}$. The resulting field profile along the magnet axis is illustrated in figure $\left[2.2^{[1]}\right.$. It is evident that the magnetic field continuously increases from the If plateau to the hf center. Therefore the relaxation times of the polarized nuclei become longer during the transfer and more polarization is preserved. In addition, the distance between the field of polarization and detection is minimized to $47 \mathrm{~cm}$. Thereby, the sample transfer is accomplished within $65 \mathrm{~ms}$.

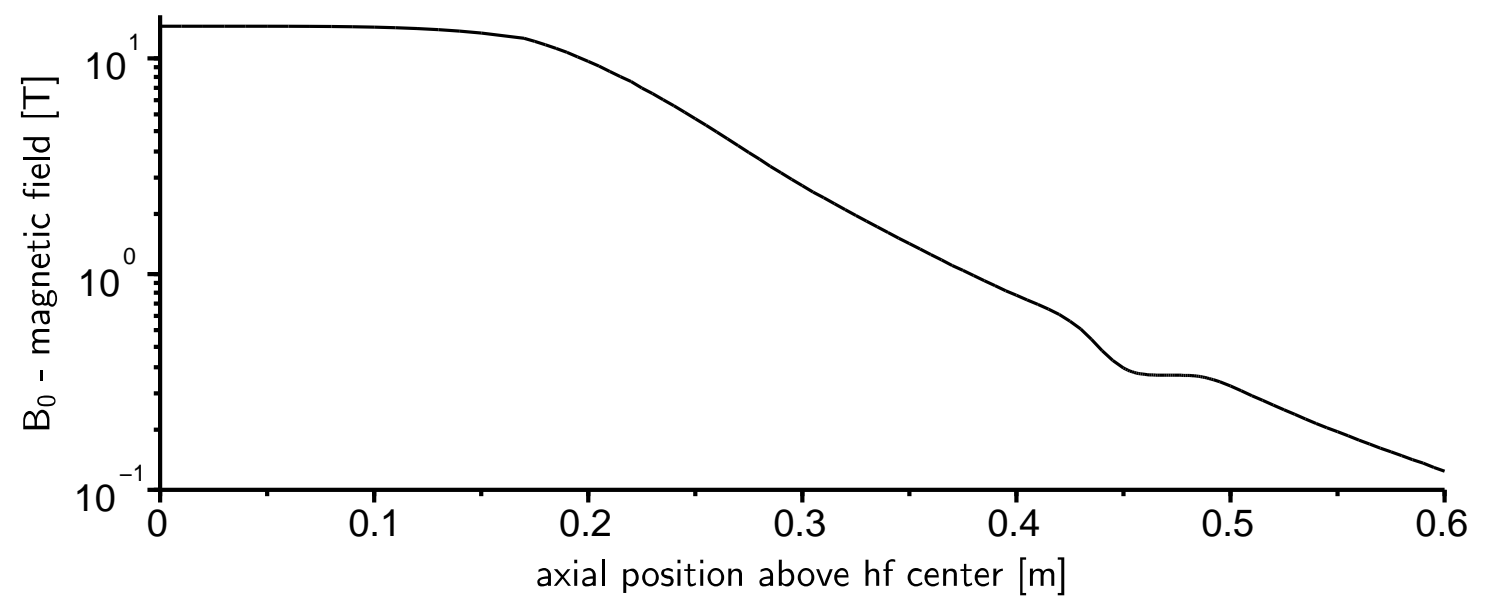

Figure 2.2.: Measured magnetic field profile along the magnet bore. The field continuously increases along the magnet axis from the ferroshim plateau to the magnetic center of the magnet. The plateau in the low field position can be adjusted by moving the ferroshim system between $0.328 \mathrm{~T}$ and $0.363 \mathrm{~T}$. 1$]$ 
The ferroshim system generates a field plateau with a length of $10 \mathrm{~mm}$ and a calculated homogeneity of $\pm 1 \mathrm{mT}$. The position of the ferroshim system can be adjusted inside the magnet bore in the range of $\pm 5 \mathrm{~mm}$ in axial direction to shift the field plateau between $0.325-0.360 \mathrm{~T}$ corresponding to electron and proton spin Larmor frequencies in the range $9.19-10.17 \mathrm{GHz}$ and $13.96-15.46 \mathrm{MHz}$, respectively.

The ferroshim system contains a sweep coil $\left(Z_{0}\right)$ and a shim coil $\left(Z_{1}\right)$ in addition to the ferromagnetic metal cylinder. The latter is driven by an electric direct current source and allows to further improve the field homogeneity at the plateau by producing an additional gradient strength of $2.82 \pm 0.02 \mathrm{mT} \mathrm{cm}^{-1} \mathrm{~A}^{-1}$. With the sweep coil, an extra field of $5.24 \pm 0.002 \mathrm{mT} \mathrm{A}^{-1}$ is superimposed on the static field. A sweep current of approximately $\pm 1 \mathrm{~A}$ is applied by the E-Scan console, resulting in a maximum spectral width of $104 \mathrm{G}(10.4 \mathrm{mT})$ over the EPR spectrum. 


\subsubsection{DNP probe}

The centerpiece of the DNP probe is the EPR cavity (figure 2.3), which operated in the $\mathrm{TM}_{110}$ mode ${ }^{[1]}$. It incorporates two modulation coils and an NMR coil for the acquisition of EPR and NMR spectra, respectively. Both are crucial for the optimization of the DNP parameters at the beginning of an experiment.

The geometry of the DNP cavity is given by the spatial restrictions of the ferroshim system, in which the DNP probe is mounted. In detail, the inner diameter of the ferroshim system is $40 \mathrm{~mm}$. After subtracting the walls, an inner diameter of $33 \mathrm{~mm}$ remains for the cavity. This diameter corresponds to a resonance frequency of $11.1 \mathrm{GHz}$ for a classical cylindrical cavity in the $\mathrm{TM}_{110}$ mode. Therefore, two extruded rings were added to the top and bottom plate of the DNP cavity, enabling the $\mathrm{TM}_{110}$ microwave mode at a frequency of $9.6 \mathrm{GHz}$. Furthermore, two additional plates were included into the resonant volume in the longitudinal plane to maintain

Figure 2.3.: Overview of the EPR cavity ${ }^{[2]}$ - The shuttle container of the sample is inserted into the cavity from the bottom and is placed in a quartz glass tube (a) which is in the center of the cavity volume (b). Two metal guides (c) containing the NMR loop coil (not shown) are placed parallel to the center tube. Two circular grooves (d) in the top and bottom plate (e) contain the modulation coils. The direction of the magnetic field of the microwave is enforced in the longitudinal plane by two plates (f). A gas stream is applied through a jet $(\mathrm{g})$ for pneumatic shuttling of the sample at the top of the guiding tube.

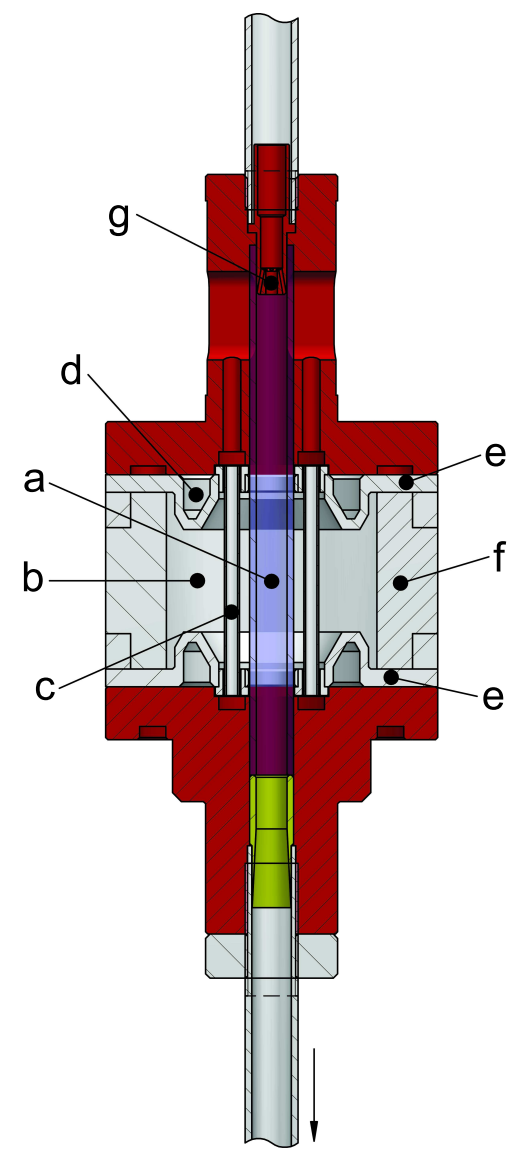



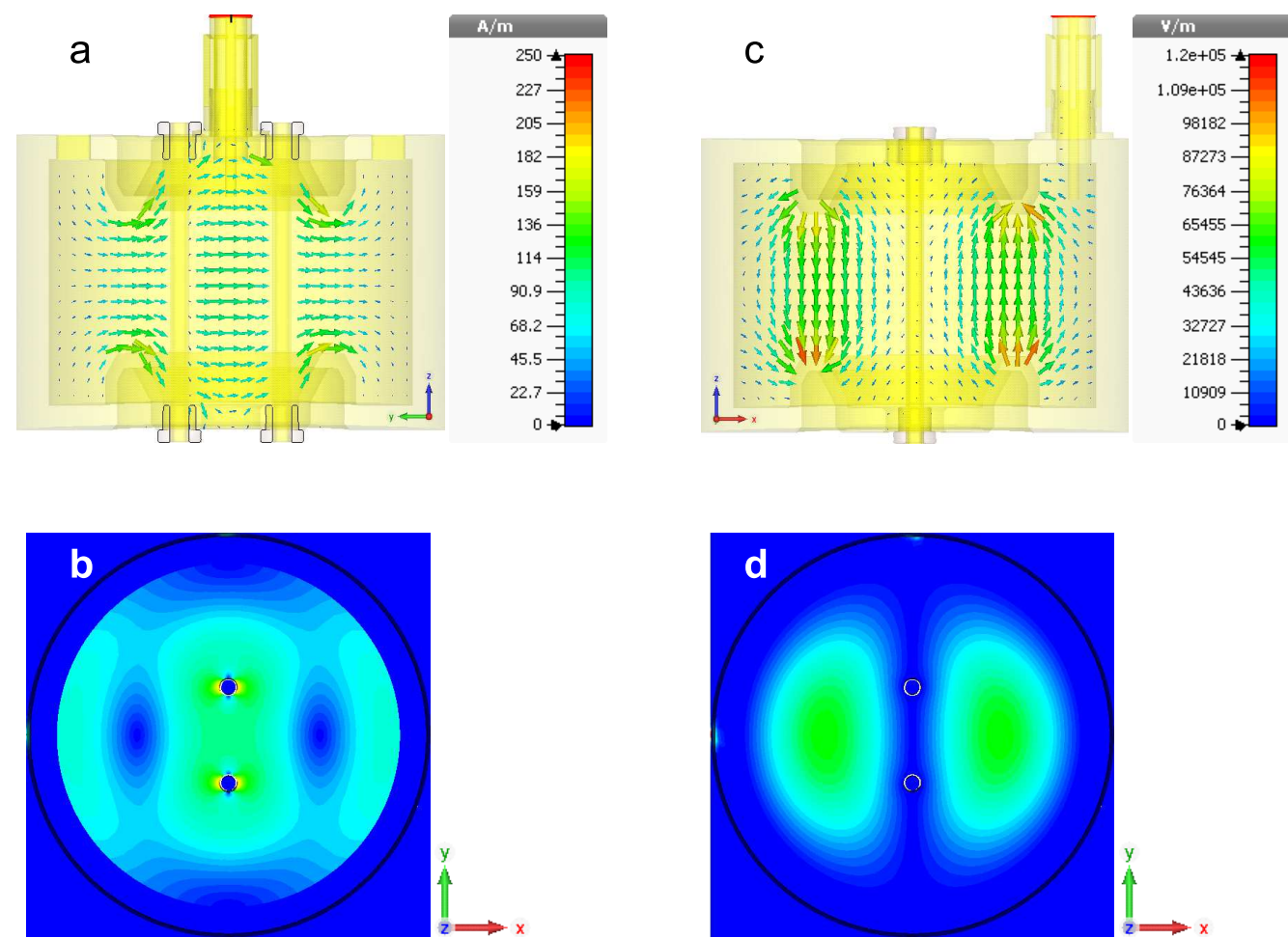

Figure 2.4.: Magnetic and electric field profile of the $\mathrm{TM}_{110}$ mode in the EPR cavity. The maximum of the transverse $B_{1}$ field is along the cavity axis - (a) longitudinal crosssection (b) transversal crosssection; The electric field vanishes in the sagittal plane - (c) longitudinal crosssection (d) transversal crosssection (Courtesy of A. Krahn, Bruker Biospin, Karlsruhe, Germany)

the correct orientation of the $\mathrm{TM}_{110}$ mode.

The $\mathrm{TM}_{110}$ mode was chosen because of a favorable microwave field distribution in regard to the sample being placed in the center along the cavity axis. On the one hand, the $\mathrm{TM}_{110}$ mode has a constant transverse magnetic field component $B_{1}$ along that axis (figure $2.4 \mathrm{a}$ ) and a maximum $B_{1}$ at the cavity center (b). On the other hand the electric field vanishes in the sagittal plane (figure $2.4 \mathrm{c}+\mathrm{d}$ ). This circumstance leads to a reduction of sample heating, which is caused by absorption of the $\mathrm{mw}$ electric field by an aqueous sample.

In addition, two isolated silver-coated tubes were placed parallel to the quartz guide in the sagittal plane (where the microwave electrical field is minimum) to guide the longitudinal sections of a multiturn wire loop used as the NMR coil. This arrangement results in transverse microwave- and rf magnetic field components that 
are orthogonal to the cavity axis. The measured $Q$ factor of the loaded cavity is 3,479 at a resonance frequency of $9.59 \mathrm{GHz}$ with a conversion factor of $\eta_{\mathrm{P}}=$ $0.055 \mathrm{mT} \mathrm{W}^{-1 / 2}$.

The temperature stability of the 1 section is a very important factor for the overall performance of the DNP set-up. Especially, a temperature rise during the DNP experiments, caused by absorbed microwave power, leads to a mechanical expansion of the cavity and of the ferroshim system in the long run. These geometric changes effect the microwave properties of the cavity and the magnetic field profile of the ferroshim. The consequence is a less efficient saturation of the EPR line due to an partially unmatched cavity and an inhomogeneous magnetic field. To prevent these effects, a Bruker cooling unit (BCU) was employed to cool the sample cavity and the ferroshim system with a constant gas flow of cold nitrogen. Thereby, not only the heating effect caused by microwave irradiation in the cavity, but also the heat dissipation from the sweep coil $\left(Z_{0}\right)$ and the shim coil $\left(Z_{1}\right)$ of the ferroshim system is compensated except for very long irradiation times $(>6 \mathrm{~s})$ at high power $(>8 \mathrm{~W})$. Furthermore, the sample heating during the DNP built-up is reduced by 10 to $15^{\circ} \mathrm{C}$. By adjusting the gas flow, the overall temperature in the lff part can be regulated. 


\subsection{Sample shuttle system}

\subsubsection{Sample shuttle container}

The design of the shuttle container is crucial for the quality of the recorded DNP spectra. Additionally, it assists maintaining a constant shim quality through out the whole experiment despite the very fast shuttling motion. However, a recurring obstacle are arsing gas bubbles in the liquid, because the sample is sealed in the shuttle container.

These problems of shim stability and bubble formation are resolved by the current container design, shown in figure 2.5. Two shuttle plugs (2.5 a) restrict the sample

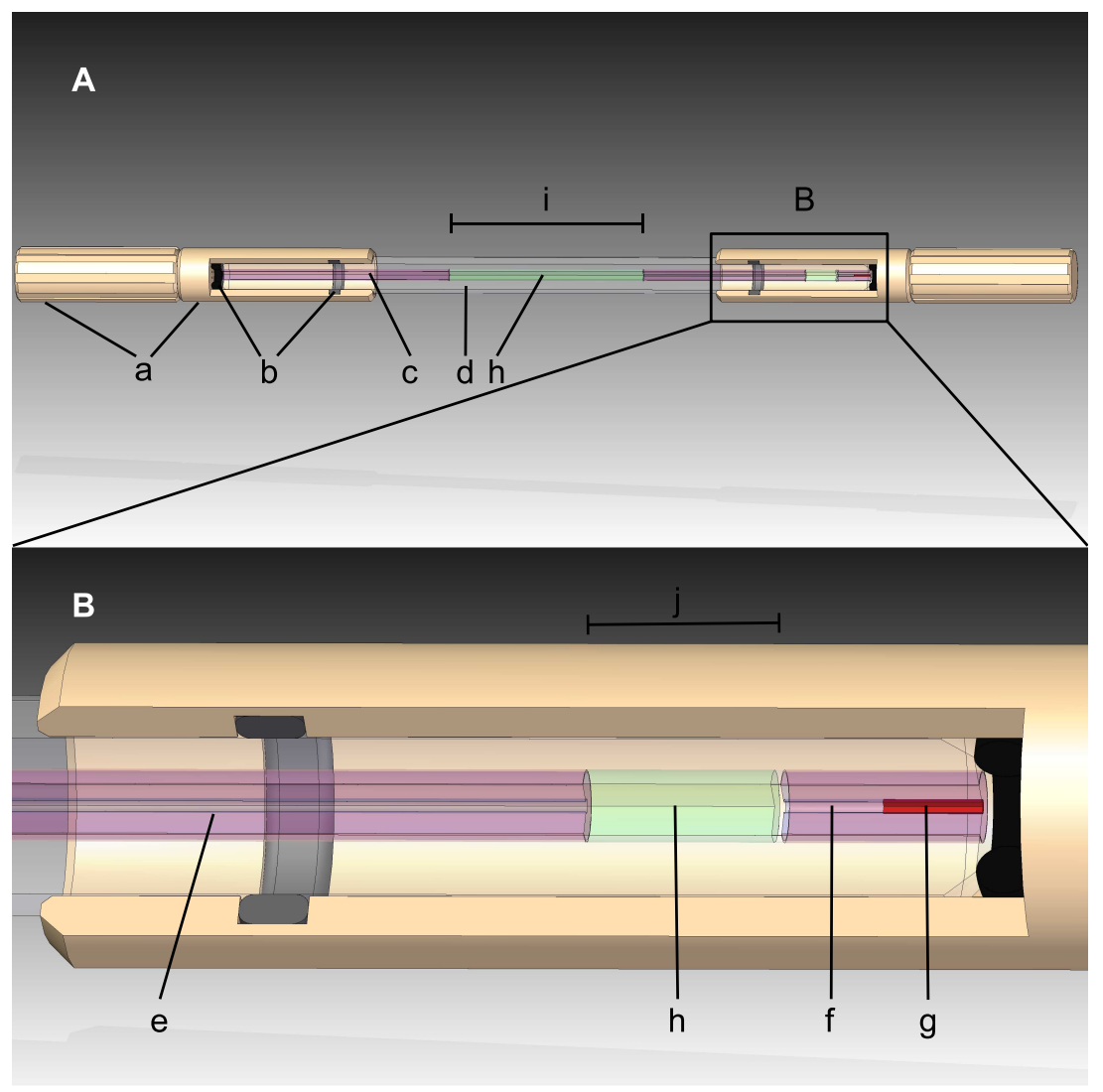

Figure 2.5.: Scheme of the shuttle container design: (A) assembled container, (B) close up of container sealing. The scheme shows a) container plug, b) vibration damper, c) glued-in glass plug, d) amorphous quartz tube, e) and f) glued-in glass capillaries, g) plug of glue, i) active sample volume $5 \mu \mathrm{L}$ and $\mathrm{j}$ ) sample reservoir/bubble collector 
container inside the probe with an accuracy of $50 \mu \mathrm{m}$. Thereby, the perturbation of the field homogeneity is very small after a shuttle cycle .

Beside the shuttle plugs, two glass capillaries (2.5 e \& f) and one glass plug (2.5) c) are inserted into the outer sample tube (2.5) d - outer diameter $3.3 \mathrm{~mm}$, inner diameter $0.75 \mathrm{~mm}$ ) and fixated in their position by UV glue. The active sample volume is restricted by the glass plug (2.5 c) and a long capillary (2.5 e), resulting in a very accurately defined sample volume. By this means, the shim is kept stable, if a rotation of the sample occurs during the shuttling process.

A small reservoir is left empty at the end of the long capillary (2.5 h), working as a sample reservoir and bubble catcher. Any remaining bubbles in the sample will not leave this volume during the shuttling process and will, therefore, not affect the shim of the active sample volume. At the further end, another, smaller capillary (2.5 f) restricts this volume. The sample container is filled with the help of a centrifuge and sealed with a cyanoacrylate-based glue or UV-glue (2.5 g), when all bubbles have been removed. The jets in the DNP probe and NMR probe as well as the end caps of the shuttle container are constructed such that a laminar stream of air is allowed to flow into the small gap between the guiding tube and the shuttle capillary. 


\subsubsection{Shuttle cycle}

A timing diagram for a typical shuttle DNP experiment (B) and for an NMR experiment $(\mathrm{A})$ is illustrated in figure 2.6. During the polarization time $t_{\mathrm{p}}$, the sample is located within the low field position in the inside of the cavity and is polarized by microwave irradiation at the electron spin Larmor frequency. The duration $t_{\mathrm{p}}$ of the microwave irradiation is determined by the polarization build-up time (see 3.3).

After the shuttle down time $t_{\mathrm{sd}}$, the sample arrives in the NMR probe and the signal is acquired after an additional post-shuttle delay $t_{\mathrm{psd}}$. In this set-up, the pneumatic shuttle system and the gate of the microwave amplifier are triggered by
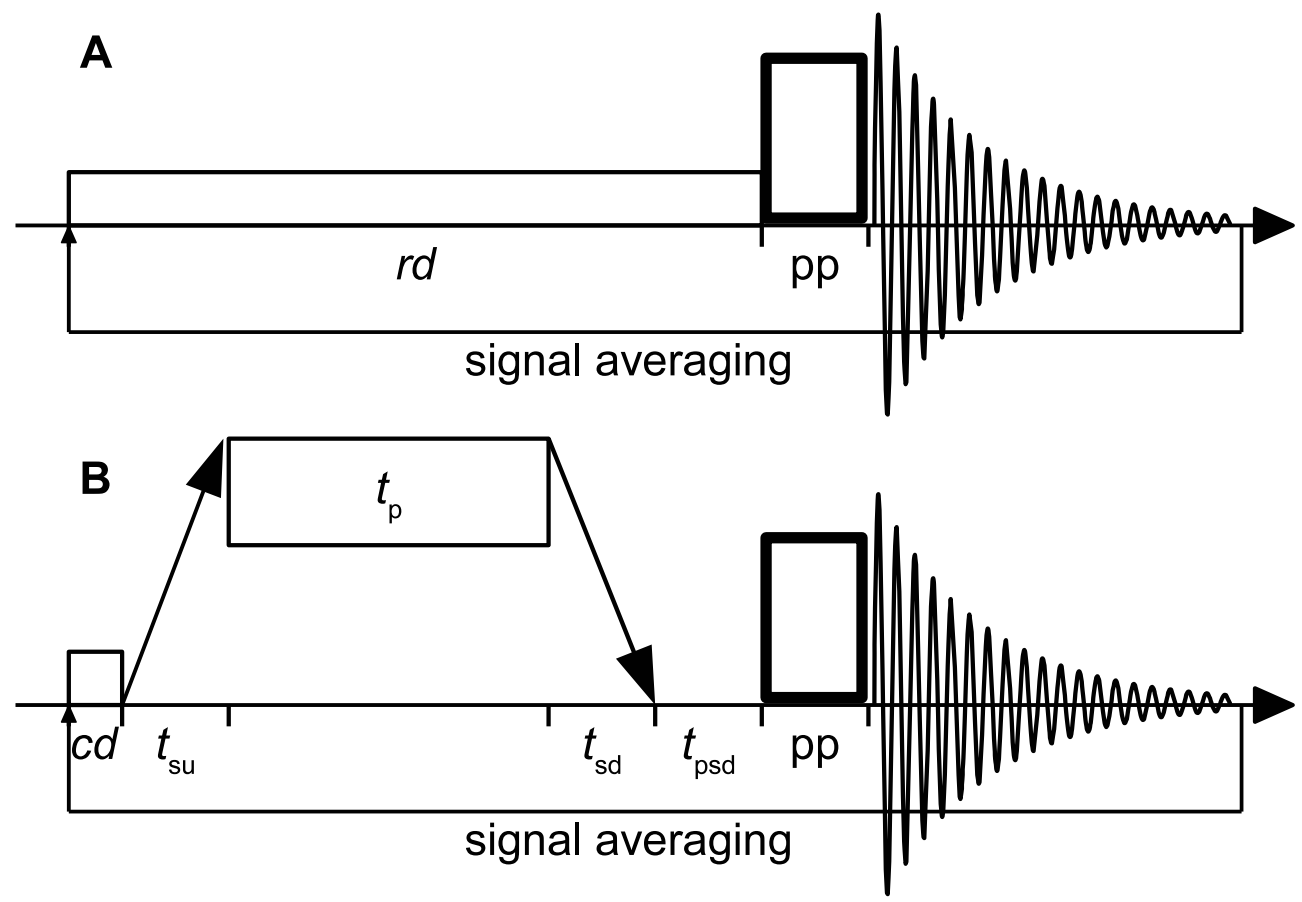

Figure 2.6.: Shuttle timing diagram: (A) NMR experiment with Boltzmann polarization. $r d$ is a recovery delay for the Boltzmann polarization followed by the pulse program (pp) and the signal acquisition. This sequence is repeated several times for signal averaging. For the DNP experiment, $r d$ is replaced by several steps: a short cooling delay $c d$ in the hfl, the sample transfer to the 【f] position during $t_{\mathrm{su}}$, the polarization time $t_{\mathrm{p}}$, during which the microwave irradiation occurs, and the sample transfer back to the hf position during the shuttle down time $t_{\mathrm{sd}}$. After a short post shuttle delay $t_{\mathrm{psd}}$ for the sample to settle in the NMR probe, the pulse program can be applied and the signal acquired. As in (A), this sequence can be repeated several times for signal averaging. 
the NMR console. Therefore, the optimum polarization time, the pre- and postshuttle delays are controlled by the pulse program. The shuttle time $t_{\mathrm{sd}}$ depends on the applied transfer pressure and was found to be approximately $50 \mathrm{~ms}$ in the experiments with a pressure reading of 4 bar. 


\subsection{NMR probe}

A high resolution probe (dual ${ }^{1} \mathrm{H}^{13} \mathrm{C}$ with ${ }^{2} \mathrm{H}$ lock channel) optimized for shuttling (figure 2.7) is used to record the NMR signal. Inside the probe, an extension of the shuttle tube towards the lower end facilitates the insertion of the sample into the shuttle system without removing the probe from the magnet. The basic mechanical design of the probe is equivalent to a standard $5 \mathrm{~mm}$ high resolution probe for which low-susceptibility and/or susceptibility compensated materials are used. This is especially important for the wire material of the NMR coil and all other components in its close vicinity. Here, the susceptibility compensation is optimized for operation under a nitrogen atmosphere. As a result, the same spectral resolution was obtained after shimming as for standard high resolution probes. The radio frequency ( $\mathrm{RF})$ circuit corresponds to that of a typical dual-probe with an inner saddle coil for ${ }^{1} \mathrm{H}$ and ${ }^{13} \mathrm{C}$ observation, and an orthogonal outer coil for an additional ${ }^{2} \mathrm{H}$ lock channel.

Due to the high speed of the shuttle container, special care has to be taken to prevent mechanical vibrations of the probe directly after the sample transfer. These could lead to noticeable artifacts in the spectrum. Therefore, the shuttle system is mechanically decoupled from the probe in order to address this problem and to achieve the necessary reproducibility for the shuttle DNP experiments. 
Figure 2.7.: Overview of the NMR probe with the glass tube (a) to position the shuttle container in the NMR coil $(b+c)$ in the magnetic center $(\mathrm{h})$. The connection to the transfer system is made via a conical adaptor (d) that is supported by rubber toroidal joints to attenuate mechanical vibrations (e). The stopper (i) for the shuttle container consists of an air jet which applies the pressure to shuttle the sample to the low-field position and provides a laminar stream of shuttle gas when the sample is in the NMR probe. An additional Z-gradient coil (g) was installed inside the probe cover (f).

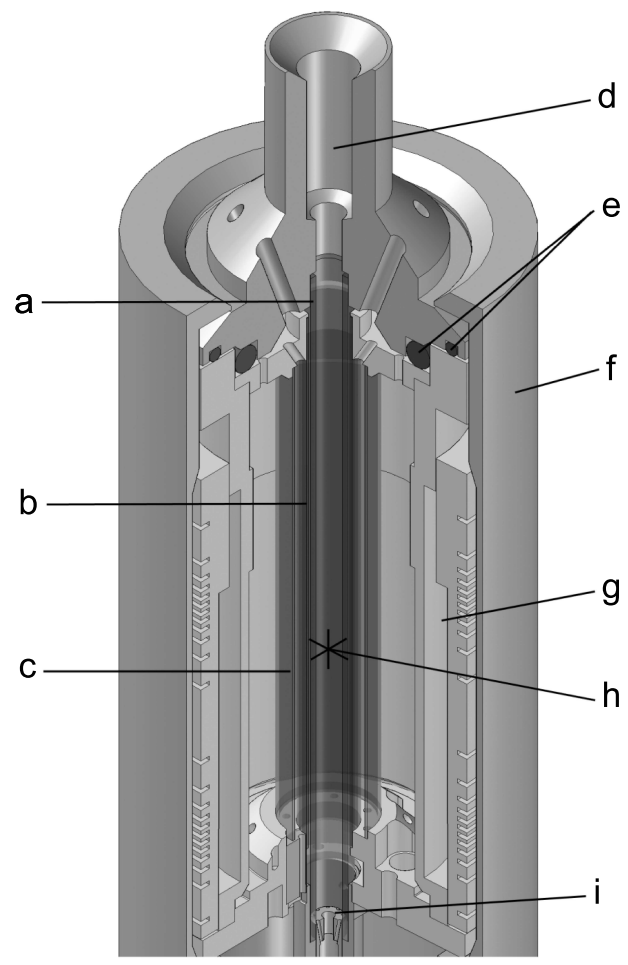





\section{Material and Methods}

\subsection{Chemical reagents and sample preparation}

L-tryptophan, L-tryptophan- $\mathrm{d}_{8},{ }^{15} \mathrm{~N}_{2},{ }^{13} \mathrm{C}_{11}$ and L-tryptophan- ${ }^{15} \mathrm{~N}_{2},{ }^{13} \mathrm{C}_{11}$ were purchased from AppliChem GmbH (Germany), Cambridge Isotope Laboratories (USA) and Euriso-Top GmbH (Germany), respectively. Furthermore, the sodium 2,2dimethyl-2-silapentane-5-sulfonate- $\mathrm{d}_{6}(\mathrm{DSS})$ as well as the unlabeled TEMPONE and ascorbic acid for the diffusion measurements were purchased from Sigma-Aldrich Chemie GmbH (Germany).

In addition, the TEMPONE- $\mathrm{d}_{16},{ }^{15} \mathrm{~N}$ for all DNP experiments was synthesized and provided by the courtesy of Dr. Andrei Leonov based on published syntheses [66,67]. The wild-type, uniformly ${ }^{15} \mathrm{~N}$ and ${ }^{13} \mathrm{C}$ labeled human ubiquitin (ubiquitin-U- ${ }^{15} \mathrm{~N}, \mathrm{U}$ ${ }^{13} \mathrm{C}-9047.47 \mathrm{~g} / \mathrm{mol}$ ) was expressed according to a previously published protocol by Johnson et al. ${ }^{[68]}$ and kindly provided by Dr. Stefan Becker and Sebastian Wolf. The protein sequence of the wild-type human ubiquitin in amino acid abbreviations is:

\section{MQIFVKTLTG KTITLEVEPS DTIENVKAKI QDKEGIPPDQ QRLIFAGKQL EDGRTLSDYN IQKESTLHLV LRLRGG}

The protein was dissolved in a buffer of $50 \mathrm{mM}$ sodium chloride and $50 \mathrm{mM}$ sodium phosphate in $\mathrm{D}_{2} \mathrm{O}$ at a $\mathrm{pH}$ of 6.5 .

Before the DNP measurements, all sample solutions were degased by ultrasonic cavitation with an UIS250v ultrasonic processor $(250 \mathrm{~W}$, frequency $24 \mathrm{kHz}$ - Hielscher Ultrasonics $\mathrm{GmbH}$ ) at an amplitude between 60 and $70 \%$. The pulse length was set to $50 \%$ for an total duration of approximately 5 min. Subsequently, the degassed samples were centrifuged (Hettich EBA3S centrifuge) at $5000 \mathrm{rpm}$ into the glass capillary of the shuttle container. After the complete removal of all gas 
bubbles from the capillary, it was sealed with ultraviolet-glue (Panacol Vitralit 2020 or 1605). The glue was cured with ultraviolet light irradiation (UV-lamp PanacolElosol UV-P280) for four times 3 min with breaks of 1 min in between. 


\subsection{Sample temperature and microwave power}

The temperature of the DNP sample was determined by the chemical shift difference between the methyl peak of DSS and the water peak in the proton spectrum. This method is based on the dependence of the chemical shift of DSS and of water on the temperature (figure [3.1), which was published by Hoffman [69].

The required $\mathrm{mw}$ power for the $\mathrm{DNP}$ experiments is generated by a $20 \mathrm{~W} \mathrm{mw}$ amplifier. The output power level of this amplifier depends on the power and frequency of the incoming $\mathrm{mw}$ signal. This signal is generated by the E-Scan console with an internal attenuation parameter for the signal power. The relation between this parameter and the $\mathrm{mw}$ output power of the amplifier was measured for three different frequencies (3.2) - 9.58 GHz (solid line) $9.595 \mathrm{GHz}$ (dashed) and $9.61 \mathrm{GHz}$ (dotted line). The presented data was recorded and provided by the courtesy of Alexander Krahn and Andreas Tavernier.

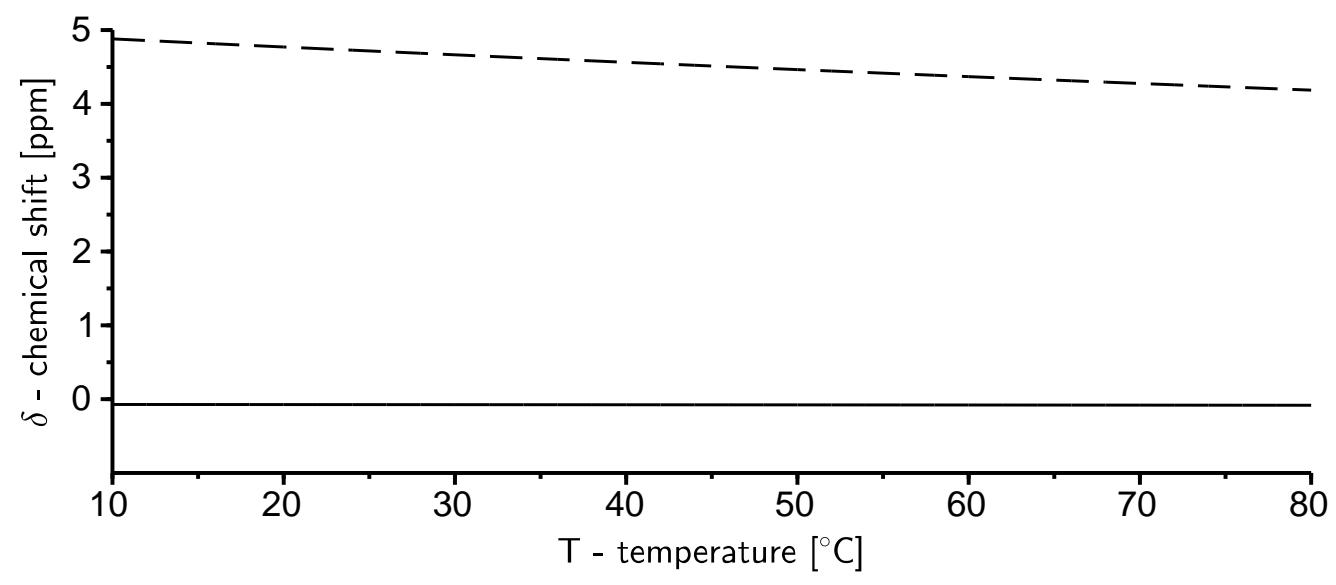

Figure 3.1.: Dependence of the chemical shift of DSS and of water on the sample temperature $[69]$. 


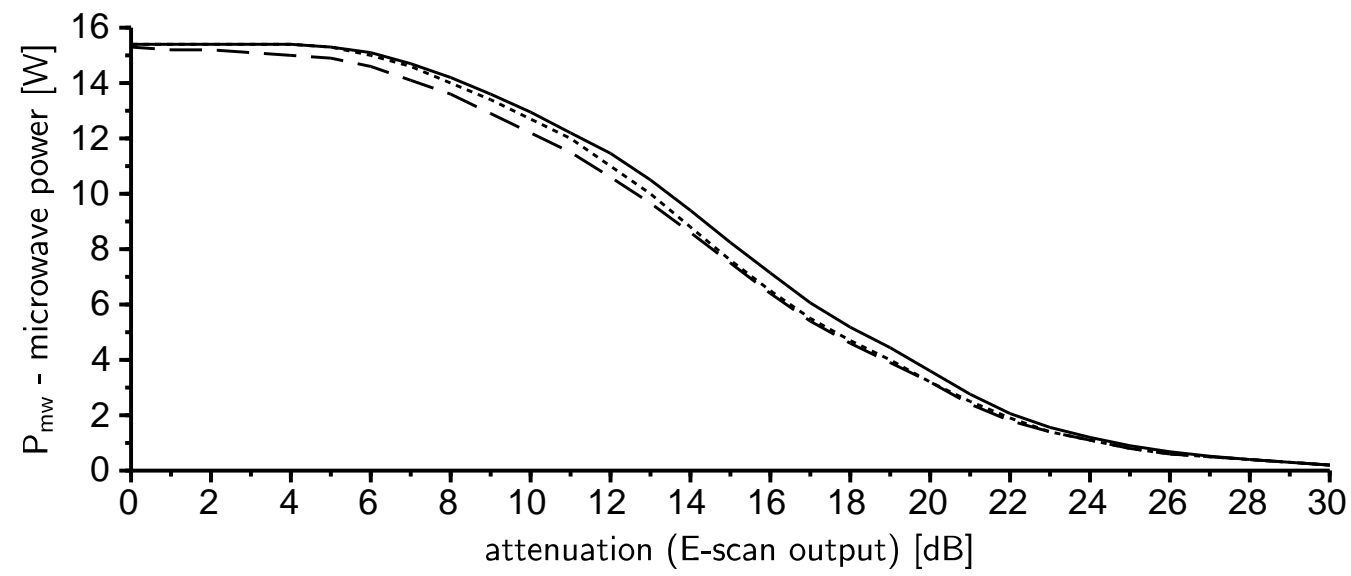

Figure 3.2.: Dependence of the output power of $\mathrm{mw}$ amplifier on the attenuation of the E-Scan mw output for different frequencies - $9.58 \mathrm{GHz}$ (solid line) $9.595 \mathrm{GHz}$ (dashed) and $9.61 \mathrm{GHz}$ (dotted line).

\subsection{NMR and DNP experiments}

\section{General remarks:}

All Boltzmann polarization and DNP experiments were conducted with the in chapter 2 described shuttle DNP spectrometer. The only exception are the measurements of the diffusion constant. These were conducted on a spectrometer (Bruker Karlsruhe, Germany) with $700 \mathrm{MHz}$ proton frequency and a TCI cryoprobe (Z-gradient).

In general, the chemical shifts are given in relation to the methyl signal of DSS which was set to $-0.074 \mathrm{ppm}$. Furthermore, the experiments with sample transfer were conducted with a maximum pneumatic shuttle pressure of 2.5 to 3.0 bar of the maximum 5 bar and a shuttle up delay $t_{\text {su }}$ of $100 \mathrm{~ms}$. The sum of the shuttle down delay and the post shuttle delay was set to 70 to $80 \mathrm{~ms}$.

$1 D$ proton experiments:

The one dimensional proton NMR experiments with Boltzmann polarization or DNP, except the measurements of the applied global enhancement factor, were conducted with the following parameters. The spectra were acquired with water suppression by the watergate pulse sequence $(\mathrm{w} 5)^{[70]}$, without deuterium locking, $12 \mathrm{k}$ data points in the time domain and a sweep width of $12 \mathrm{ppm}$. 
Furthermore, the measurements of the applied global enhancement factor were conducted by using excitation sculpting with gradients ${ }^{[71]}$ for the water suppression. In the experiment with Boltzmann polarization, 3214 data points were acquired with a spectral width of $12 \mathrm{ppm}$. The experiment with DNP was conducted with 3214 acquired data points and a spectral width of $12 \mathrm{ppm}$. For the optimization of the experimental parameters, $T_{2}^{*}$ was obtained by measuring the linewidth at half height $L$ and the following equation:

$$
T_{2}^{*}=\frac{1}{\pi L}
$$

For this purpose, a spectrum with $32 \mathrm{k}$ data points was measured to ensure a sufficient resolution for the determination of the linewidth.

\section{Relaxation and polarization build-up measurements:}

The build-up curve of the dynamic nuclear polarization was measured by systematically increasing $t_{\mathrm{p}}$ at a fixed $P_{\mathrm{MW}}$. Thereby, the build-up time $T_{1, \mathrm{bu} \text {,lf }}$ could be determined by fitting the build-up with a mono-exponantial function. The spin lattice relaxation times in the low field, $T_{1, \text { lf }}$, and in the high field, $T_{1, \mathrm{hf}}$, were measured by the decay of the DNP. For this purpose, a relaxation delay was introduced after $t_{\mathrm{p}}$ in the low field and after $t_{\mathrm{psd}}$ in the high field. The obtained signal decay was fitted with a simple mono-exponential function to extract $T_{1}$. For the measurement

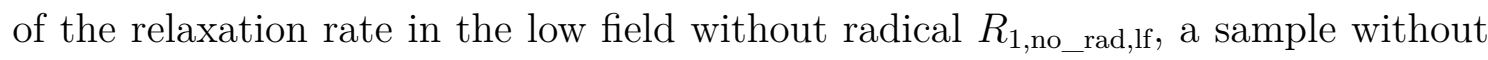
the addition of the radical was shuttled with the Boltzmann polarization of the highfield to the low-field position. After a relaxation delay, the sample was transferred back to the high-field position and the remaining signal was detected. The resulting curve with various relaxation delays was fitted with a mono-exponential function.

\section{Diffusion ordered NMR spectroscopy (DOSY):}

In order to obtain the diffusion constant of TEMPONE- $\mathrm{d}_{16},{ }^{15} \mathrm{~N}$, a sample solution with $5 \mathrm{mM}$ unlabeled TEMPONE, $5 \mathrm{mM}$ DSS and $5 \mathrm{mM}$ ascorbic acid in $\mathrm{D}_{2} \mathrm{O}$ was prepared. The ascorbic acid reduces the radical ${ }^{[72]}$ and, thereby, it enables the detection of the methyl protons of TEMPONE in an NMR experiment.

For the DOSY experiment, a standard Bruker pulse program (stegp1s) with a 
stimulated echo ${ }^{[73]}$ sequence was employed. The gradient strength was linearly increased from 2 to $95 \%$ in 20 steps in a pseudo-2D experiment with 16k acquired data points and a sweep width of $10 \mathrm{ppm}$ in the direct dimension. A gradient strength of $100 \%$ corresponds to $54.34 \mathrm{G} / \mathrm{cm}$. The dependence of the signal amplitude on the gradient strength was fitted by an exponential function to obtain the diffusion constant $\underline{[73]}$.

\section{$1 D$ carbon experiments:}

The one dimensional carbon spectra were acquired with $2 \mathrm{k}$ data points, $4 \mathrm{k}$ scans and a spectral width of $180 \mathrm{ppm}$. The experiments were conducted with deuterium locking due to a measurement time of several hours. For this purpose, additional delays were implemented in the pulse sequence, during which the deuterium locking was activated and at its end deactivated. The acquired Free Induction Decays (FID) were multiplied by an exponential window function with $20 \mathrm{~Hz}$ line broadening.

\section{${ }^{1} \mathrm{H}^{13} \mathrm{C}$-Heteronuclear Single Quantum Coherence $(\mathrm{HSQC})$}

The ${ }^{1} \mathrm{H}-{ }^{13} \mathrm{C}-\left[\mathrm{HSQC}\right.$ spectra ${ }^{[13]}$ were recorded with a 3-9-19 watergate ${ }^{[74]}$ and without deuterium locking. Therefore, a selective $90^{\circ}$ pulse was applied to the DSS signal of the sample before the first INEPT (Insensitive Nuclei Enhanced by Polarization Transfer) step of the HSQC. This results in a positive and constant DSS signal in all transients of the two dimensional spectrum, which is used for chemical shift referencing.

HSQC spectra were recorded with $4 \mathrm{k}$ complex data points in the direct dimension and 128 complex data points in the indirect dimension. All spectra were processed using NMRPipe. At first, the direct dimension was Fourier transformed and the phase adjusted. Subsequently, the chemical shift of all transients was corrected based on the chemical shift of DSS with a software macro of NMRPipe . Afterwards, the data was inverse Fourier transformed. For the final processing of the spectra, only 512 data points of the direct dimension were used. A sine bell window function with an offset of 0.5 , automatic zero-filling and an automatic baseline correction was applied to both dimensions.

Software for data analysis 
All one dimensional NMR experiments and the DOSY experiment were processed with Topspin 3.2 (Bruker BioSpin). For the processing of the ${ }^{1} \mathrm{H}_{-}{ }^{13} \mathrm{C}-\mathrm{HSQC}$ spectra, the program NMRPipe ${ }^{[75]}$ and, for the assignment, the program CARA (www.cara.nmr.ch) ${ }^{[76]}$ were used. The program Igor Pro 6.2.2.2 (Wave-Metrics) was used for the fitting of the experimental data. The calculations related to the coupling factor were conducted with the program Scilab 5.4.1 (Scilab Enterprises S.A.S). 



\section{Results and discussion}

\subsection{DNP signal enhancement of protons}

After the installation and comprehensive testing of the major set-up improvements, namely the generally overhauled DNP probe, active cooling of the If section and the newly designed shuttle container, DNP measurements with L-tryptophan were conducted. As an amino acid, it occurs in proteins and therefore represents an



Figure 4.1.: ${ }^{1} \mathrm{H}$ DNP enhancement of L-tryptophan $(10 \mathrm{mM})$ in $\mathrm{D}_{2} \mathrm{O}(99.8 \%)$ with TEMPONE- $\mathrm{d}_{16}{ }^{15} \mathrm{~N}$ (10 mM) and DSS (5 mM; not shown) as a chemical shift reference. The spectrum was acquired with $t_{\mathrm{p}}=3 \mathrm{~s}, P_{\mathrm{MW}}=12.5 \mathrm{~W}, 64$ scans and a sample volume of about $5 \mu \mathrm{L}$. The sample temperature was $52{ }^{\circ} \mathrm{C}$ (initial temperature $25^{\circ} \mathrm{C}$ ). A watergate pulse sequence (w5) was used for water suppression ${ }^{[70]}$. The values of $\varepsilon_{\mathrm{hf} \text {,max }}$ are given at the signals. 
Table 4.1.: Measured signal enhancement of L-tryptophan and DSS after $t_{\mathrm{p}}=1 \mathrm{~s}$ and $P_{\mathrm{MW}}=9 \mathrm{~W}$ - The sample temperature reached $42{ }^{\circ} \mathrm{C}$ due to the microwave irradiation. The initial temperature was $25^{\circ} \mathrm{C}$. By measuring $T_{1, \text { ff }}$ and $T_{1, \mathrm{hf}}$ (see refmnm), the lower and upper limit for the potential relaxation losses $(\lambda)$ during $t_{\mathrm{sd}}$ and $t_{\mathrm{psd}}$ could be calculated as well as the range of $\varepsilon_{\mathrm{hf}, \mathrm{id}}$

\begin{tabular}{cccccc}
\hline & $\varepsilon_{\mathrm{hf}}$ & $T_{1, \mathrm{lf}}[\mathrm{s}]$ & $T_{1, \mathrm{hf}}[\mathrm{s}]$ & $\lambda[\%]$ & $\varepsilon_{\mathrm{hf}, \mathrm{id}}$ \\
\hline \hline $\mathrm{H}^{\alpha}$ & -1.0 & 0.35 & 0.60 & $22-32$ & -1.3 to $-1.5 \pm 0.2$ \\
$\mathrm{H}^{\beta 2}$ & -1.1 & 0.23 & 0.31 & $36-43$ & -1.7 to $-1.9 \pm 0.2$ \\
$\mathrm{H}^{\beta 3}$ & -1.1 & 0.24 & 0.34 & $34-41$ & -1.6 to $-1.9 \pm 0.3$ \\
$\mathrm{H}^{\delta 1}$ & -1.7 & 0.28 & 0.45 & $23-33$ & -2.2 to $-2.6 \pm 0.2$ \\
$\mathrm{H}^{\zeta 2}$ & -1.8 & 0.26 & 0.40 & $26-34$ & -2.4 to $-2.8 \pm 0.2$ \\
$\mathrm{H}^{\varepsilon 3}$ & -1.9 & 0.27 & 0.44 & $24-33$ & -2.5 to $2.8 \pm 0.2$ \\
$\mathrm{H}^{\zeta 3}$ & -1.9 & 0.26 & 0.42 & $24-33$ & -2.5 to $-2.9 \pm 0.3$ \\
$\mathrm{H}^{\eta 2}$ & -2.2 & 0.25 & 0.43 & $23-33$ & -2.8 to $-3.3 \pm 0.3$ \\
DSS H & -1.7 & 0.36 & 0.58 & $19-26$ & -2.1 to $-2.4 \pm 0.1$ \\
\hline
\end{tabular}

interesting molecule for testing. Its molecular weight is comparable to D-glucose, for which signal enhancements have already been achieved ${ }^{[1]}$. TEMPONE- $\mathrm{d}_{16}{ }^{15} \mathrm{~N}$ was used as a polarizer in the DNP experiments based on findings from previous experiments $[1,47,77]$. Furthermore recent results had shown that this polarizing agent reaches higher saturation factors at lower concentrations than other possible radicals (i.e. Frémy's salt ${ }^{[58]}$ ).

The DNP enhancements measured for the protons of L-tryptophan are illustrated in figure 4.1. The radical concentration was $10 \mathrm{mM}$ in $\mathrm{D}_{2} \mathrm{O}$. The overall enhancement factor $\varepsilon_{\text {hf }}$ as measured at high field ranges from -1.0 to -2.4 . The methyl groups of DSS (not shown in this figure) were enhanced by a factor of -2.0. These findings are in agreement with previously published results ${ }^{[1]}$. The polarization time $t_{\mathrm{p}}$ was set to $3 \mathrm{~s}$ and the microwave power $P_{\mathrm{MW}}$ amounted to $12.5 \mathrm{~W}$. These parameters ensured that the irradiated EPR line splitting by hyperfine coupling is saturated and that the polarization build-up reaches saturation.

A drawback of the high microwave power and the long polarization time is severe sample heating caused by absorption of microwave radiation by the water molecules. The sample temperature rose from an initial value of $25^{\circ} \mathrm{C}$ to a final value of $52{ }^{\circ} \mathrm{C}$. Adjusting $P_{\mathrm{MW}}$ to $9 \mathrm{~W}$ and $t_{\mathrm{p}}$ to $1 \mathrm{~s}$ reduced the heating and gave a final temperature of $42{ }^{\circ} \mathrm{C}$. The resulting enhancement factors are shown in table 4.1. The obtained 
enhancements are slightly smaller than for the longer irradiation time, potentially due to an incomplete saturation of the electron transition, the short irradiation time itself, or less heating. The latter is most probable, as the enhancement factor and the longitudinal nuclear relaxation times increase with temperature $\underline{[50,52]}$. This results in a slightly smaller leakage factor and smaller relaxation losses during the shuttle process. Since these losses amount about $30 \%$, even small changes in the relaxation time may have a strong effect on the measured enhancement in the highfield position. The precise magnitude of these losses will be calculated in detail below (see 4.3). 


\subsection{Global proton enhancement}

For a better evaluation of the DNP enhancement factors achieved with the DNP setup described above (chapter 2), it is necessary to expand the definition of the enhancement factor, since there are more parameters to a NMR experiment than the obtainable nuclear polarization. The signal accumulation rate, line broadening or bleaching by the radical and other potential factors influence the sensitivity. For this reason, Vitzthum et al. ${ }^{[78]}$ introduced in solid state DNP the global enhancement factor $\varepsilon_{\text {global }}$. In a first step this factor was transferred to the context of liquid state shuttle DNP and in a second step refined to the individual parameters of a liquid state NMR experiment with the aim of determining the gain in SNR per measurement time.

\subsubsection{Introducing the global enhancement factor}

The global enhancement factor $\varepsilon_{\text {global }}$ introduced by Vitzthum et al. ${ }^{[78]}$ is defined in the following way:

$$
\varepsilon_{\text {global }}=\varepsilon_{\mathrm{DNP}} \varepsilon_{\text {dilution }} \varepsilon_{\text {bleach }} \sqrt{\kappa}
$$

$\varepsilon_{\mathrm{DNP}}$ is the enhancement factor in reference to the thermal Boltzmann polarization. For the shuttle DNP setup, this parameter is identical to $\varepsilon_{\mathrm{hf}} \cdot \varepsilon_{\text {dilution }}$ is a parameter designed for solid state DNP experiments and is 1 in this case, as the sample is already dissolved by definition. $\varepsilon_{\text {bleach }}$ takes differences between the signal amplitudes with and without added radical into account under the same experimental conditions.

Finally, $\kappa$ is the ratio between the $\mathrm{DNP}$ polarization build-up time, $T_{1, \mathrm{bu} \text {,lf }}$ and the spin lattice relaxation time without radical, $T_{1, \mathrm{hf}}$ :

$$
\kappa=\frac{T_{1, \mathrm{hf}}}{T_{1, \mathrm{bu}, \mathrm{lf}}}
$$

If $T_{1, \mathrm{bu} \text {,lf }}$ is shorter than $T_{1, \mathrm{hf}}$, the total experimental time per scan becomes shorter using shuttle DNP. Therefore, the SNR is increased by faster signal accumulation. This increase in SNR gives an enhancement in addition to the DNP enhancement and is proportional to $\sqrt{\kappa}$. In the shuttle DNP spectrometer, the polarization takes 
Table 4.2.: The $\mathrm{DNP}$ enhancement factors at $52{ }^{\circ} \mathrm{C}$ (initial temperature $25^{\circ} \mathrm{C}$ ) for $\mathrm{L}-$ tryptophan are used to calculate the global enhancement factor (equation 4.1). $\varepsilon_{\text {bleach }}$ was directly taken from the measured intensity drop of the L-tryptophan proton signals in the presence of the radical.

\begin{tabular}{ccccccc}
\hline & $\varepsilon_{\text {hf,max }}$ & $\varepsilon_{\text {bleach }}$ & $T_{1, \mathrm{hf}}[\mathrm{s}]$ & $T_{1, \mathrm{bu}, \mathrm{lf}}[\mathrm{s}]$ & $\sqrt{k}$ & $\varepsilon_{\text {global }}$ \\
\hline \hline $\mathrm{H}^{\alpha}$ & -1.0 & 0.47 & 2.7 & 0.43 & 2.5 & $-1.2 \pm 0.2$ \\
$\mathrm{H}^{\beta 2}$ & -1.4 & 0.57 & 0.8 & 0.39 & 1.4 & $-1.1 \pm 0.2$ \\
$\mathrm{H}^{\beta 3}$ & -1.2 & 0.49 & 0.8 & 0.34 & 1.5 & $-0.9 \pm 0.2$ \\
$\mathrm{H}^{\delta 1}$ & -1.9 & 0.57 & 6.7 & 0.43 & 3.9 & $-4.2 \pm 0.7$ \\
$\mathrm{H}^{\zeta 2}$ & -1.9 & 0.58 & 2.7 & 0.38 & 2.7 & $-2.9 \pm 0.4$ \\
$\mathrm{H}^{\varepsilon 3}$ & -2.0 & 0.51 & 4.6 & 0.38 & 3.5 & $-3.7 \pm 0.6$ \\
$\mathrm{H}^{\zeta 3}$ & -2.0 & 0.58 & 2.7 & 0.40 & 2.6 & $-3.1 \pm 0.4$ \\
$\mathrm{H}^{\eta 2}$ & -2.4 & 0.52 & 2.7 & 0.38 & 2.6 & $-3.3 \pm 0.4$ \\
\hline
\end{tabular}

place at low magnetic fields in the presence of a radical, which leads to much shorter build-up times $T_{1, \text { bu,lf }}$ compared to the spin lattice relaxation times without the radical at high fields.

The calculated global enhancement factors for L-tryptophan are shown in table 4.2. For the aromatic protons, $\varepsilon_{\text {global }}$ is up to two times higher than $\varepsilon_{\mathrm{hf}}$, reaching a value of -4.2 for one of the aromatic protons. Conversely, the aliphatic protons cannot be enhanced and show only small differences in $\varepsilon_{\text {hf }}$. Furthermore, $\varepsilon_{\text {global }}$ of $\mathrm{H}^{\beta 2}$ and $\mathrm{H}^{\beta 3}$ is even smaller than $\varepsilon_{\mathrm{hf} \text { max }}$. One reason for these different results is found in $\sqrt{\kappa}$. As all protons show a similar $T_{1, \text { bu,lf }}$, yet diverse values of $T_{1, \mathrm{hf}}, \sqrt{\kappa}$ covers the range from 1.4 to 3.9 for these different nuclei.

An important reduction of $\varepsilon_{\text {global }}$ originates in $\varepsilon_{\text {bleach }}$ that has a value of 0.4-0.6. This reduction is in most cases, more than compensated by $\sqrt{\kappa}$ leading to a global enhancement after all.

\subsubsection{Applied global enhancement factor}

The previously described approach of the global enhancement factor by Vitzthum et al. ${ }^{[78]}$ helped to gain a first insight into the possibilities and drawbacks of shuttle DNP, yet it is not possible to directly transfer these results to an actual NMR experiment and to directly translate them into an increased SNR.

The reason for this is an incomplete consideration of several practical aspects of 
the signal acquisition, especially in regard to the individual time per scan of the DNP experiments compared to the Boltzmann polarization experiments. Therefore the global enhancement factor was adjusted to obtain the best comparability between these two experiments with the additional aim to base it on the SNR per unit time. By this means, the enhancement was linked to the actual measurement time of the experiments. In the following, this approach is described in more detail. An applied global enhancement factor is given for an example proton of L-tryptophan and compared to an experiment.

In a first step, the acquisition time must be adjusted to the experimental conditions of each respective sample for the optimal SNR per unit measurement time since the transverse relaxation rate $R_{2}$ is increased by the presence of the radical in the solution of the DNP sample. Thereby, the free induction decay (FID) of the NMR signal becomes shorter, but the noise level during the acquisition is unaltered. This is illustrated by the relation between the signal intensity and the acquisition time $t_{\text {acqu }}{ }^{[79]}$

$$
\text { Signal } \propto \int_{0}^{t_{\text {acqu }}} e^{-t R_{2}} d t
$$

as well as the relation between noise level and the acquisition time

$$
\text { Noise } \propto \sqrt{t_{\text {acqu }}}
$$

The noise level is independent of $R_{2}$. By solving equation 4.3 and dividing the result by equation 4.4, the dependency of the signal to noise ratio on the acquisition time is given by

$$
S / N\left(t_{\text {acqu }}\right)=a\left(\frac{1-e^{-t_{\text {acqu }} R_{2}}}{R_{2} \sqrt{t_{\text {acqu }}}}\right)
$$

with a proportionality factor $a$. The numerical solution of equation 4.5 gives a maximum $\underline{\text { SNR }}$ for $t_{\text {acqu }}$ at $1.26 R_{2}^{-1}[\underline{[9]}$. With this equation, the enhancement factor for the signal acquisition $\varepsilon_{\text {acqu }}$ is described by

$$
\varepsilon_{\mathrm{acqu}}=\frac{S / N_{\mathrm{DNP}}\left(t_{\mathrm{acqu}, \mathrm{DNP}}\right)}{S / N_{\mathrm{BZM}}\left(t_{\mathrm{acqu}, \mathrm{BZM}}\right)}=\sqrt{\frac{R_{2, \mathrm{BZM}}}{R_{2, \mathrm{DNP}}}}
$$

The proportionality constant $a$ is identical for both types of experiments under 
the condition that the acquisition time of the DNP and Boltzmann polarization experiment are both adjusted to the transverse relaxation rate and that the only difference in the experimental set-up is the presence or absence of the radical.

$\varepsilon_{\text {acqu }}$ incorporates the changes in $R_{2}$ caused by the radical and the resulting the line broadening. Therefore, it replaces in the global enhancement the previously introduced $\varepsilon_{\text {bleach }}$ describing the signal intensity difference.

Furthermore, the replacement of $\sqrt{\kappa}$ is necessary, as it is only proportional to the SNR gain attained by an increased acquisition rate. It does not give an accurate value for the increased SNR per unit time in an actual experiment. In the previous model of the global enhancement factor, only the build-up times were used for $\kappa$ (see equation 4.2). Yet the shuttle DNP specific delays such as $c d, t_{\mathrm{su}}, t_{\mathrm{sd}}$ and $t_{\mathrm{psd}}$ must be considered as well, since they contribute to the overall experimental time alongside the acquisition time.

A more accurate approach for the acquisition rate enhancement $\varepsilon_{\text {rate }}$ is based on the number of scans $n s$ performed in the two compared experiments. As the SNR increases with the square root of $n s, \varepsilon_{\text {rate }}$ is defined as

$$
\varepsilon_{\text {rate }}=\sqrt{\frac{n s_{\mathrm{DNP}}}{n s_{\mathrm{BZM}}}}
$$

where $n s_{\text {DNP }}$ is the number of scans of the $\mathrm{DNP}$ experiment and $n s_{\mathrm{BZM}}$ of the Boltzmann polarization experiment. These numbers can be calculated by using the experimental parameters. This gives

$$
\begin{aligned}
& n s_{\mathrm{DNP}}=\frac{t_{\mathrm{mt}}}{t_{\mathrm{p}}+t_{\mathrm{acqu}, \mathrm{DNP}}+t_{\mathrm{pp}, \mathrm{DNP}}}-d s \\
& n s_{\mathrm{BZM}}=\frac{t_{\mathrm{mt}}}{r d+t_{\mathrm{acqu}, \mathrm{BZM}}+t_{\mathrm{pp}, \mathrm{BZM}}}-d s
\end{aligned}
$$

with the polarization time $t_{\mathrm{p}}$ for a $\mathrm{DNP}$ experiment and the recovery delay $r d$ for the Boltzmann polarization experiment as illustrated in figure 2.6. $t_{\mathrm{pp}}$ represents the residual pulse program duration of one single scan without $t_{\mathrm{p}}, r d$ and $t_{\mathrm{acqu}}$, yet it includes $c d, t_{\mathrm{su}}, t_{\mathrm{sd}}, t_{\mathrm{psd}}$, the $\mathrm{RF}$ pulses of the pulse sequence, etc. The overall measurement time $t_{\mathrm{mt}}$ and the number of dummy scans are identical for the DNP 
and the Boltzmann polarization experiment to maintain comparability.

In a concluding step, an optimization of $t_{\mathrm{p}}$ and $r d$ based on the experimental parameters is needed for a maximum SNR per unit time. These parameters include properties of the samples, such as relaxation times, as well as experimental delays, such as shuttle times or dummy scans. The relation between the SNR per unit time $S / N_{\mathrm{t}}$ and the polarization build-up time $t_{\mathrm{BU}}$ is based on the exponential build-up of the nuclear polarization and the square root of the total number of scans, during the measurement time $t_{\mathrm{mt}}$ :

$$
S / N_{\mathrm{t}} \propto I_{\infty}\left(1-e^{-R_{1} t_{\mathrm{BU}}}\right) \sqrt{n s}
$$

Here $I_{\infty}$ represents the maximum polarization achievable for an indefinite polarization time. For a DNP experiment, the polarization build-up time $t_{\mathrm{BU}}$ corresponds to $t_{\mathrm{p}}$, but for an experiment with Boltzmann polarization, it represents the sum of the acquisition time $t_{\text {acqu }}$ and the recovery delay $r d$, as the recovering of the thermal equilibrium starts right after the end of the pulse sequence. After including equation 4.8 and a proportionality factor $b$, the equations for the SNR per unit time in $\mathrm{DNP}$ and Boltzmann polarization experiments are given by

$$
S / N_{\mathrm{t}, \mathrm{DNP}}=b I_{\infty, \mathrm{DNP}}\left(1-e^{-R_{1, \mathrm{BU}, \mathrm{lf}} t_{\mathrm{p}}}\right) \sqrt{\frac{t_{\mathrm{mt}}}{t_{\mathrm{p}}+t_{\mathrm{pp}}+t_{\mathrm{acqu}}}-d s}
$$

and

$$
S / N_{\mathrm{t}, \mathrm{BZM}}=b I_{\infty, \mathrm{BZM}}\left(1-e^{-R_{1, \mathrm{hf}}\left(r d+t_{\mathrm{acqu}}\right)}\right) \sqrt{\frac{t_{\mathrm{total}}}{r d+t_{\mathrm{pp}}+t_{\mathrm{acqu}}}-d s}
$$

, respectively. These equations incorporate a high number of individual experimental parameters, such as $t_{\mathrm{pp}}$ or $d s$. This makes it necessary to solve equation 4.11 and 4.12 numerically for each specific measurement in order to obtain the optimal polarization time $t_{\mathrm{p}, \mathrm{opt}}$ and the optimal recovery delay $r d_{\mathrm{opt}}$ for the highest SNR per unit measurement time. These two values lead to different percentages of the maximum polarization $I_{\infty}$ per scan:

$$
\frac{I_{\mathrm{opt}, \mathrm{DNP}}}{I_{\infty, \mathrm{DNP}}}=1-e^{-R_{1, \mathrm{BU}, \mathrm{lf}} t_{\mathrm{p}, \mathrm{opt}}}
$$




$$
\frac{I_{\mathrm{opt}, \mathrm{BZM}}}{I_{\infty, \mathrm{BZM}}}=1-e^{-R_{1, \mathrm{hf}}\left(r d_{\mathrm{opt}}+t_{\mathrm{acqu}}\right)}
$$

$I_{\mathrm{opt}, \mathrm{DNP}}$ and $I_{\mathrm{opt}, \mathrm{BZM}}$ are the polarizations obtained with $t_{\mathrm{p}, \mathrm{opt}}$ and $r d_{\mathrm{opt}}$, respectively. Since the ratios of equation 4.13 and 4.14 are quite different, they give rise to the new element $\varepsilon_{\text {build-up }}$ of the globe enhancement factor:

$$
\varepsilon_{\text {build-up }}=\frac{I_{\mathrm{opt}, \mathrm{DNP}} I_{\infty, \mathrm{BZM}}}{I_{\infty, \mathrm{DNP}} I_{\mathrm{opt}, \mathrm{BZM}}}
$$

Finally, the new applied global enhancement factor is given by multiplying all previously described partial enhancement factors:

$$
\varepsilon_{\text {app }}=\varepsilon_{\text {DNP }} \varepsilon_{\text {build-up }} \varepsilon_{\text {rate }} \varepsilon_{\text {acqu }}
$$

In order to test and illustrate the previously derived applied global enhancement factor, an experimental setting was defined. Two almost identical samples with $50 \mathrm{mM}$ L-tryptophan and $10 \mathrm{mM}$ DSS in $\mathrm{D}_{2} \mathrm{O}$ were prepared, differing only in the addition of $10 \mathrm{mM}$ TEMPONE- $\mathrm{d}_{16}{ }^{15} \mathrm{~N}$ as polarizer for the DNP experiment. Subsequently, a ${ }^{1} \mathrm{H}$ spectrum with optimized parameters was recorded for both samples with shuttle $\mathrm{DNP}$ and Boltzmann polarization, respectively. The overall measurement time of each experiment was $t_{\mathrm{mt}}=30 \mathrm{~min}$. This time included $16 \mathrm{ds}$, conducted before the signal acquisition. In addition, the acquisition parameters were optimized for the applied global enhancement factor.

Since these optimizations are based on single spin properties, such as longitudinal

Table 4.3.: Calculated applied global enhancement factor of the $\mathrm{H}^{\delta 1}$ of L-tryptophan for a measurement time of 30 min together with the parameters which were used for the calculation.

\begin{tabular}{cccccccc}
\hline & $L[\mathrm{~Hz}]$ & $R_{2}^{*}\left[\mathrm{~s}^{-1}\right]$ & $t_{\mathrm{acqu}}[\mathrm{s}]$ & $t_{\mathrm{pp}}[\mathrm{s}]$ & $t_{\mathrm{p}, \mathrm{opt}}[\mathrm{s}]$ & $r d_{\mathrm{opt}}[\mathrm{s}]$ & $n s$ \\
\hline \hline BZM & 1.8 & 5.7 & 0.22 & 0.04 & - & 7.4 & 218 \\
DNP & 2.8 & 8.8 & 0.14 & 0.28 & 0.8 & - & 1452 \\
\hline
\end{tabular}

\begin{tabular}{cccccc}
$\varepsilon_{\text {acqu }}$ & $\varepsilon_{\text {rate }}$ & $\varepsilon_{\text {build-up }}$ & $\varepsilon_{\text {DNP }}$ & $\varepsilon_{\text {app,th }}$ & $\varepsilon_{\text {app,exp }}$ \\
\hline \hline 0.8 & 2.6 & 1.2 & -1.9 & -4.7 & -4.0 \\
\hline
\end{tabular}


and transverse relaxation time, the $\mathrm{H}^{\delta 1}$ of L-tryptophan was chosen as an example nucleus. This proton is the most promising nucleus of L-tryptophan due to its relaxation properties.

For the process of optimization, the linewidth $L$ of the $\mathrm{H}^{\delta 1}$ peak was used to calculate the transverse relaxation rate $R_{2}^{*}$ :

$$
L=\frac{R_{2}^{*}}{\pi}
$$

$R_{2}^{*}$ describes the FID most accurately by including the field inhomogeneity which remained after shimming.

The measured linewidth and the resulting transverse relaxation rate of the DNP and the Boltzmann polarization (BZM) experiments are listed in table 4.3, With these rates and equation 4.6, the acquisition time $t_{\text {acqu }}$ and the enhancement factor for the signal acquisition $\varepsilon_{\text {acqu }}$ was calculated. With a value of 0.8 , it reflects the line broadening induced by the presence of the radical and the accompanying effect of a decreased SNR. Therefore, to be exact, it is not a true enhancement factor, but a diminishment factor.

After setting up all parameters of the pulse sequences including preliminary values for $t_{\mathrm{p}}$ and $r d$, the duration of $t_{\mathrm{pp}}$ was determined. Subsequently $t_{\mathrm{p}, \mathrm{opt}}$ and $r d_{\mathrm{opt}}$ were calculated numerically based on equations 4.11 and 4.12, respectively, with $t_{\mathrm{pp}}$, the longitudinal relaxation times from table 4.2 and the acquisition time $t_{\text {acqu }}$. During this calculation, the number of scans was determined for each experiment, as well.

Furthermore, a rate enhancement $\varepsilon_{\text {rate }}$ of 2.6 was calculated. Based on equation 4.13 and 4.14, $t_{\mathrm{p}, \mathrm{opt}}$ and $r d_{\mathrm{opt}}$ lead for each scan to 68 and $84 \%$ of the maximum polarization, respectively. Therefore, the achieved build-up enhancement $\varepsilon_{\text {build-up }}$ is 1.2 .

Multiplication of all the partial enhancement factors (equation 4.16) gives a theoretical applied global enhancement factor $\varepsilon_{\text {app,th }}$ of -4.7 for $\mathrm{H}^{\delta 1}$. In the corresponding measurements, an experimental applied global enhancement factor $\varepsilon_{\text {app,exp }}$ of -4.0 in respect to the SNR per measurement time was achieved, which is shown in figure 4.2. This value of -4.0 is close to the calculated value. The discrepancy could be attributed to the difficulty of keeping all the parameters of the DNP and Boltzmann polarization experiments identical. The most challenging parameters were the 


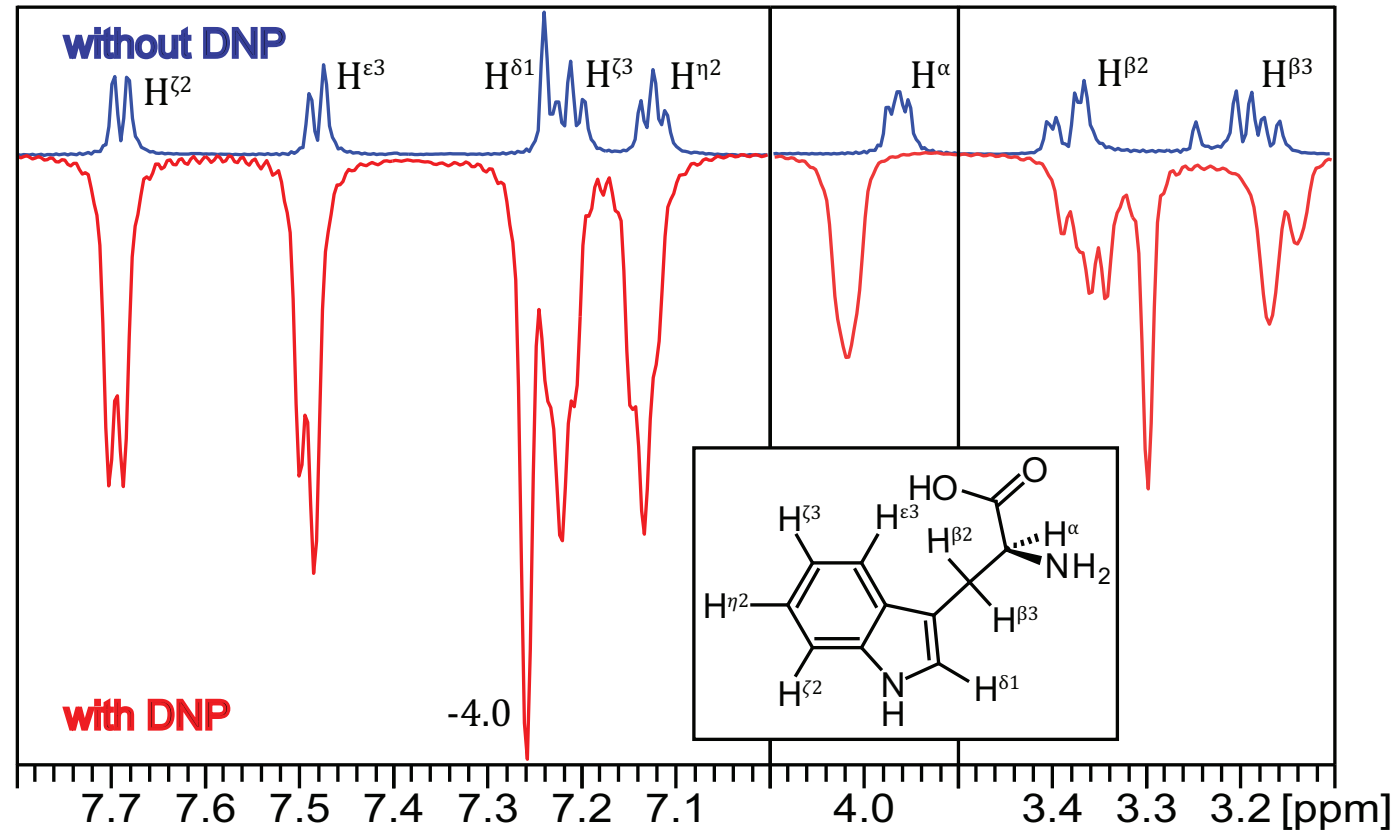

Figure 4.2.: Applied global enhancement factor of $\mathrm{H}^{\delta 1}$ of L-tryptophan

temperature and the shim quality during the DNP measurement.

One obvious benefit of the applied global enhancement factor is that it is a directly observable experimental result. Its focus is on the application of shuttle DNP and the gain of SNR per measurement time. Therefore, it makes the evaluation of the capabilities of the shuttle DNP spectrometer more reliable. However, it is limit to the experiment, for which it was optimized, and its determination is very intricate. The optimization was solely done for one proton of L-tryptophan. If all protons are included, a compromise must be found for the polarization build-up time and the acquisition time since each proton has different relaxation properties.

In addition, by focusing on the maximum SNR per unit time, the aspect of resolution is completely neglected. This becomes obvious, if one examines the spectral resolution in figure 4.2. Some of the multiplets in the $\mathrm{DNP}$ experiment are not resolved, even though it would be generally possible with this set-up as figure 4.1 demonstrates. The for SNR optimized acquisition time is too short to provide enough spectral resolution for the multiplets. Further consequences are baseline distortions around the signals due to an incomplete recorded FID.

In conclusion, the applied global enhancement factor allows for making predic- 
tions for expected enhancement, yet only if all experimental parameters and spin properties are known. Generalized statements about the enhancement are barely possible. Nevertheless, the one fact, that becomes obvious by looking at the parameters and gained enhancements is, that spins with long relaxation times (longitudinal and transverse) will benefit the most by shuttle DNP. 


\subsection{The coupling factor}

The coupling factor is the crucial element of the DNP experiment, since it limits how much polarization is transferred from the electrons to the nuclei. Therefore, it is very important to understand the transfer mechanism and the influencing parameters to ensure the optimal experimental conditions. In the following subsections the coupling factor is calculated for the protons of L-tryptophan. Subsequently, the translational diffusion model and the combined model are applied to the system of TEMPONE- $\mathrm{d}_{16},{ }^{15} \mathrm{~N}$ and L-tryptophan in $\mathrm{D}_{2} \mathrm{O}$ and assessed based on the experimental data in order to determine, which model describes the intermolecular spin-spin interaction best.

\subsubsection{Calculation of the coupling factor}

Previously in liquid state DNP, only the coupling factor of water molecules was calculated $[50,80,81]$. Here, for the first time, the coupling factor of an dissolved molecule was calculated with the help of experimental data. For this purpose, each parameter of equation 1.26 was calculated, except for the saturation factor, which was taken from the literature $\underline{[57]}$.

Table 4.4.: $R_{1, \text { no_rad,lf }}$ and $R_{1, \text { rad,lf }}\left(1 / T_{1, \text { lf }}\right.$ of table4.1) were used to calculate the leakage factor for each proton of both L-tryptophan and the DSS methyl groups using equation 1.27 - By using table 4.1 and equation 1.26, the coupling factors were determined. Because $\varepsilon_{\mathrm{hf}, \text { id }}$ was estimated for a certain range of values, the coupling factors are presented as ranges as well. The sample temperature increased to $42{ }^{\circ} \mathrm{C}$ (initial temperature $25^{\circ} \mathrm{C}$ ).

\begin{tabular}{ccccc}
\hline & $R_{1, \text { no_rad,lf }}\left[\mathrm{s}^{-1}\right]$ & $R_{1, \text { rad,lf }}\left[\mathrm{s}^{-1}\right]$ & $f$ & $\xi$ \\
\hline \hline $\mathrm{H}^{\alpha}$ & 0.72 & 2.90 & $0.75 \pm 0.03$ & 0.13 to $0.14 \pm 0.03$ \\
$\mathrm{H}^{\beta 2}$ & 1.67 & 4.38 & $0.62 \pm 0.04$ & 0.20 to $0.23 \pm 0.03$ \\
$\mathrm{H}^{\beta 3}$ & 1.66 & 4.13 & $0.60 \pm 0.06$ & 0.20 to $0.23 \pm 0.03$ \\
$\mathrm{H}^{\delta 1}$ & 0.22 & 3.60 & $0.94 \pm 0.01$ & 0.18 to $0.20 \pm 0.03$ \\
$\mathrm{H}^{\zeta 2}$ & 0.50 & 3.91 & $0.87 \pm 0.01$ & 0.21 to $0.23 \pm 0.03$ \\
$\mathrm{H}^{\varepsilon 3}$ & 0.51 & 3.70 & $0.86 \pm 0.02$ & 0.21 to $0.24 \pm 0.03$ \\
$\mathrm{H}^{\zeta 3}$ & 0.54 & 3.78 & $0.86 \pm 0.02$ & 0.22 to $0.24 \pm 0.03$ \\
$\mathrm{H}^{\eta 2}$ & 0.45 & 4.01 & $0.89 \pm 0.01$ & 0.23 to $0.27 \pm 0.03$ \\
DSS H & 0.36 & 2.75 & $0.87 \pm 0.01$ & 0.18 to $0.20 \pm 0.03$ \\
\hline
\end{tabular}


For equation 1.26, it is necessary to calculate the relaxation losses $\lambda$ which occur during the shuttling delay $t_{\mathrm{sd}}$ and the delay after the sample arrival $t_{\mathrm{psd}}$ and which diminish the measured high-field enhancement. Without these relaxation losses, one has the ideal enhancement in the high-field position $\varepsilon_{\mathrm{hf}, \mathrm{id}}$. To obtain this, a monoexponential polarization decay was presumed. The longitudinal nuclear relaxation time during the shuttle process (during $t_{\mathrm{sd}}$ ) is defined by the relaxation time $T_{1, \mathrm{ff}}$ in the low field as the lower limit and the relaxation time $T_{1, \mathrm{hf}}$ in the high field as the upper limit. The magnetic field increases continuously while moving the sample rapidly from the low-field to the high-field position inside the magnet bore. During the post-shuttle delay $t_{\mathrm{psd}}$, the enhancement decays with $T_{1, \mathrm{hf}}$ in the high field.

Based on these presumptions, a lower and an upper limit for the signal enhancement without relaxation losses $\varepsilon_{\text {hf,id }}$ was calculated as well as the relaxation losses $(\lambda)$ themselves. A summary of the calculated losses and the ideal high-field enhacnement is presented in table 4.1. The relaxation losses range from 19 to $43 \%$.

In the next step, the leakage factor was calculated using equation 1.27$]^{[6,77]} . R_{1, \text { rad,lf }}$ and $R_{1, \text { no_rad,lf }}$ correspond to the relaxation rates in the low-field with and without addition of the radical, respectively. The resulting leakage factors are shown in table 4.4. The aliphatic protons clearly exhibit a smaller leakage factor, which results from a higher relaxation rate without radical.

The saturation factor was determined by Türke et al. ${ }^{[57]}$ using a pulsed ELDOR experiment. Due to identical experimental conditions, a similar magnitude is expected for the setup described here. Since the saturation factor is a property of the nitroxide radical, its value is identical for all enhanced nuclei in the sample. For a solution containing $10 \mathrm{mM}$ TEMPONE- $\mathrm{d}_{16},{ }^{15} \mathrm{~N}$ a saturation factor of 0.85 was measured ${ }^{[57]}$. The two remaining factors in equation [1.26, namely the ratio between the high and the low field and the ratio between the gyromagnetic ratios of the electron and the proton, are known values and remain constant throughout the experiments with $\left(B_{0, \text { hf }} / B_{0, \text { lf }}\right)=41.5$ and $\left(\gamma_{\mathrm{S}} / \gamma_{\mathrm{I}}\right)=-658$, respectively.

Using these values and equation 1.26, it was possible to calculate the coupling factor $\xi$. Since $\mathrm{D}_{2} \mathrm{O}$ was used as a solvent, polarization transfer between the solvent and L-tryptophan did not influence the enhancement and therefore was neglected. The lower and upper limit of $\varepsilon_{\mathrm{hf}, \mathrm{id}}$ yields a lower and upper boundary for the coupling factor (table 4.4). 
All determined coupling factors are within an average range of $0.21-0.23$, with the exception of $\mathrm{H}^{\alpha}$, which shows a distinctly smaller coupling factor of 0.13-0.14. This reduced value indicates, that this proton is less accessible for the radical due to the bulky carboxyl, amino and indole group in close proximity. The reduced accessibility leads to a longer minimum distance between the unpaired electron in the radical molecule and the proton, directly resulting in a weaker coupling $[\underline{50,60}-62,81]$. The greater coupling factor of water, $\xi=0.37$ at $45^{\circ} \mathrm{C}[50]$, serves as a further indication for this distance dependence. In comparison, water molecules are significantly smaller than the L-tryptophan molecules and therefore move much closer to the unpaired electron of the radical for the polarization transfer to happen.

This evidence leads to the hypothesis, that the distance of closest approach has a strong influence on the coupling factor. Therefore, the distances between the unpaired electron and the nuclei are calculated with translational diffusion model and the combined model in the following subsection. The obtained distances are included in the assessment of these two models.

\subsubsection{Calculation of the distance of closest approach}

As described before in the previous subsection, the accessibility of the nucleus presumably influences the coupling factor via the distance of closest approach of the radical. Therefore, the translational diffusion model and the combined model (section (1.3) are used to calculate the distance of closest approach for the experimentally obtained coupling factors of L-tryptophan. In order to assess the two models, the autorelaxation rate $\rho$ is calculated as well. The rate was obtained from experimental data by the following equation, which was extracted from equation 1.27 .

$$
\rho=R_{1, \text { rad }}-R_{1, \text { no_rad }}
$$

The \relaxation rate with radical $R_{1 \text {,rad }}$ and without radical $R_{1, \text { no_rad }}$ were already used for the calculation of the leakage factor (table 4.4).

For reasons of clarity only three representative protons were selected for these calculations: The well exposed aromatic $\mathrm{H}^{\eta 2}$, the less accessible $\mathrm{H}^{\alpha}$ and the $\mathrm{H}^{\delta 1}$ as an intermediate between the previous two extremes. 
Table 4.5.: Calculated parameters of TEMPONE- $\mathrm{d}_{16},{ }^{15} \mathrm{~N}$ and L-tryptophan in $\mathrm{D}_{2} \mathrm{O}$ at $42{ }^{\circ} \mathrm{C}$.

\begin{tabular}{cccc}
\hline \multicolumn{2}{c}{ TEMPONE- ${ }_{16}{ }^{15} \mathrm{~N}$} & \multicolumn{3}{c}{ L-tryptophan } \\
$D_{\mathrm{S}}\left[10^{-10} \mathrm{~m}^{2} \mathrm{~s}^{-1}\right]$ & $\tau_{\mathrm{c}, \mathrm{S}}\left[10^{-12} \mathrm{~s}\right]$ & $D_{\mathrm{I}}\left[10^{-10} \mathrm{~m}^{2} \mathrm{~s}^{-1}\right]$ & $\tau_{\mathrm{c}, \mathrm{I}}\left[10^{-12} \mathrm{~s}\right]$ \\
\hline \hline 8.87 & 8.20 & 8.19 & 17.53 \\
\hline
\end{tabular}

Furthermore it is generally assumed, that the distance $r$ between $\vec{I}$ and $\vec{S}$ (equation 1.43) in the rotational diffusion model is almost identical to the distance of closest approach $d$ in the translational diffusion model and therefore $r$ is substituted by $d$ in all following calculations.

For the translational diffusion model, the diffusion constants of L-tryptophan and TEMPONE- $\mathrm{d}_{16},{ }^{15} \mathrm{~N}$ are needed. The diffusion constant of L-tryptophan in $\mathrm{H}_{2} \mathrm{O}$ at $25{ }^{\circ} \mathrm{C}$ was taken from L. G. Longsworth ${ }^{[82]}$. The diffusion constant of

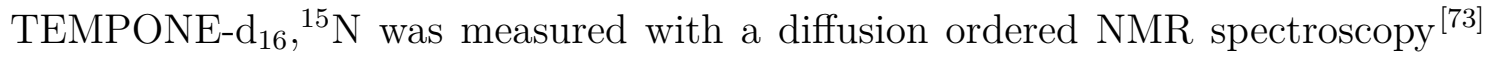
experiment (see section 3.3) on reduced unlabeled TEMPONE in $\mathrm{D}_{2} \mathrm{O}$ at $25^{\circ} \mathrm{C}$. Both diffusion constants were scaled with the Stokes-Einstein equation for translational diffusion to the experimental conditions of $42{ }^{\circ} \mathrm{C}$ and $\mathrm{D}_{2} \mathrm{O}$ as solvent:

$$
D=\frac{k_{\mathrm{B}} T}{6 \pi \eta r_{\text {hydro,trans }}}
$$

$r_{\text {hydro,trans }}$ is the hydrodynamic radius of the diffusing particle. The viscosity $\eta$ was calculated with the empirical equation by Cho et al. ${ }^{[83]}$ for the temperature dependency of the viscosity of $\mathrm{D}_{2} \mathrm{O}$.

Likewise, the parameters of the rotational diffusion model must be determined, even though these are partially more difficult to access. The rotational correlation time $\tau_{\mathrm{r}}$ between the nucleus and the unpaired electron depends amongst others on the complex life time $\tau_{\mathrm{M}}$. One may assume, that L-tryptophan is still able to rotate unhindered in the complex since only weak interactions between TEMPONE- $\mathrm{d}_{16}{ }^{15} \mathrm{~N}$ and L-tryptophane facilitate the inner sphere complex. Examples for these interactions would be a shared solvent cage and Van der Waals interactions between the molecular surfaces.

Therefore, its rotational correlation time $\tau_{\mathrm{c}, \mathrm{I}}$ limits the interactions between the 
spins and represents a reasonable approximation for the effective complex life time. Based on this, $\tau_{\mathrm{M}}$ is replaced in equation 1.41 by the rotational correlation time of L-tryptophan $\tau_{\mathrm{c}, \mathrm{I}}$ to obtain the intermolecular rotational correlation time $\tau_{\mathrm{r}}$.

The rotational correlation time of TEMPONE- $\mathrm{d}_{16},{ }^{15} \mathrm{~N}$ with $\tau_{\mathrm{c}, \mathrm{S}}=9$ ps at $30{ }^{\circ} \mathrm{C}$ and L-tryptophan with $\tau_{\mathrm{c}, \mathrm{I}}=21.8 \mathrm{ps}$ at $25^{\circ} \mathrm{C}$ are reported in the literature $\underline{[84,85]} . \tau_{1, \mathrm{~S}}$ for TEMPONE- $\mathrm{d}_{16}{ }^{15} \mathrm{~N}\left(5 \mathrm{mM}\right.$ in $\left.\mathrm{H}_{2} \mathrm{O}\right)$ measured by Türke et al. ${ }^{[57]}$ is with $298 \mathrm{~ns}$ at room temperature about four magnitudes longer than the rotational correlation times $\tau_{\mathrm{c}, \mathrm{I}}$ and $\tau_{\mathrm{c}, \mathrm{S}}$. Therefore its contribution to the intermolecular rotational correlation time $\tau_{\mathrm{r}}$ is negligible.

$\tau_{\mathrm{c}, \mathrm{S}}$ and $\tau_{\mathrm{c}, \mathrm{I}}$ were scaled by the Stokes-Einstein relation for rotational diffusion to the experimental conditions in the same manner as the diffusion constants:

$$
\tau_{\mathrm{c}}=\frac{1}{6 D_{\mathrm{rot}}}=\frac{4 \pi \eta r_{\text {hydro,rot }}^{3}}{3 k_{\mathrm{B}} T}
$$

$r_{\text {hydro,rot }}$ is the hydrodynamic radius of the rotational diffusion*. All calculated parameters are summarized in table 4.5 .

For the last missing parameter $n$, the temporal average number spins in the coordination sphere of the radical, three different values $(n=0.0001, n=0.001$ and $n=0.01)$ were tested in a first appraisal of the combined model. The distance of closest approach and the auto relaxation rate were calculated for the translational diffusion model by inserting the equations for the transition probabilities 1.31, 1.32 and 1.33 with the equations for the spectral density 1.37, 1.38 and the proportionality factor 1.39 in the equation of the coupling factor 1.34 and of the autorelaxation rate 1.14. For the same calculations with the combined model, equation 1.44 was used for the coupling factor and equation 1.14 with the transition probabilities of the inner and outer sphere relaxation. The corresponding transition probabilities (equations 1.31, 1.32 and 1.33) were obtained with the equations for the spectral density of the outer sphere relaxation 1.37 and 1.38 with the corresponding proportionality factor (equation 1.39) as well as for the spectral density of the inner sphere

*Although $r_{\text {hydro,rot }}$ is based on the Stokes-Einstein equation, too, it is different from $r_{\text {hydro,trans }}$. For L-tryptophan and TEMPONE- $\mathrm{d}_{16},{ }^{15} \mathrm{~N} / r_{\text {hydro,trans }}$ is bigger than $r_{\text {hydro,rot }}$. This is presumably the case due to a hydration shell which especially effects the hydrodynamic radius of the translational diffusion. 


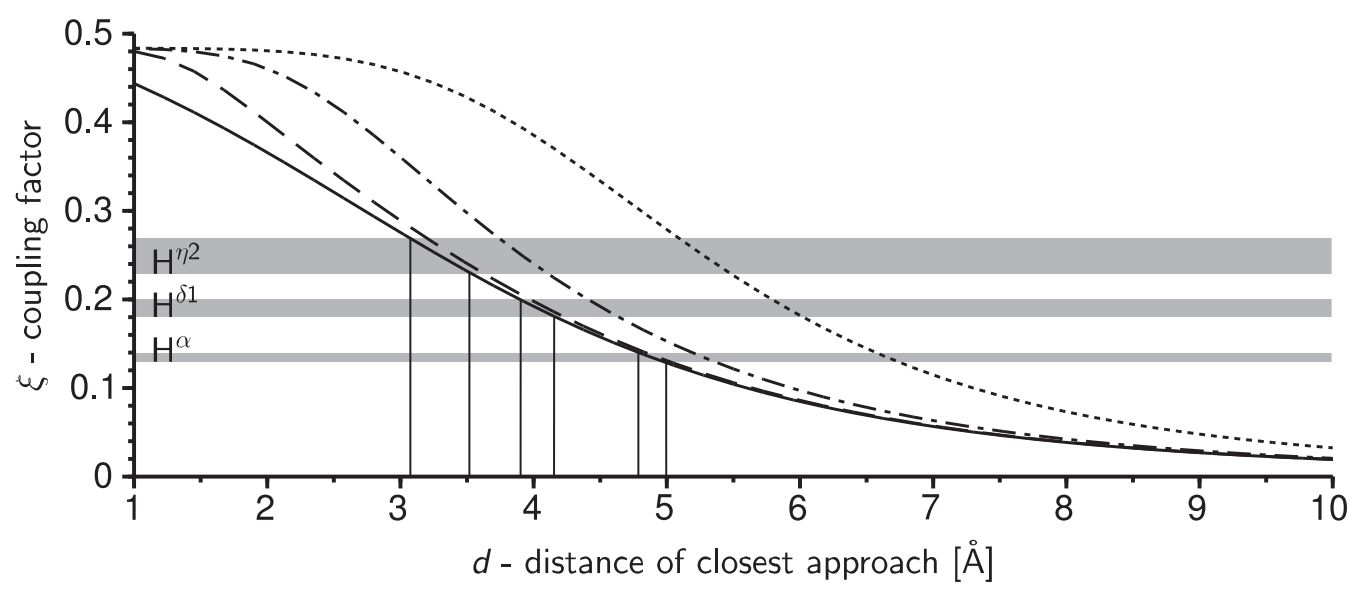

Figure 4.3.: Dependency of the coupling factor on the distance of closest approach for L-tryptophan $(10 \mathrm{mM})$ and TEMPONE- $\mathrm{d}_{16}{ }^{15} \mathrm{~N}$ in $\mathrm{D}_{2} \mathrm{O}$ at $42{ }^{\circ} \mathrm{C}$. The solid line represents the translational diffusion model $(n=0)$ and the other three lines the combined model with rotational and translational diffusion with $n=0.0001$ (dashed), $n=0.001$ (dashdotted) and $n=0.01$ (dotted).

relaxation 1.37 with the corresponding proportionality factor (equation 1.43).

The dependency of the coupling factor on the distance of closest approach is illustrated in figure 4.3 including three curves of the combined model for the different values of $n$ (dashed: $n=0.0001$, dash-dotted: $n=0.001$ and dotted: $n=0.01$ ). The translational diffusion model mathematically corresponds to $n=0$. From figure 4.3 it becomes obvious, that the coupling factor significantly increases with increasing $n$ for small distances.

Based on the corresponding range of the coupling factor of each proton, taken from table 4.4, the distance of closest approach was numerically calculated for the three example protons $\mathrm{H}^{\alpha}, \mathrm{H}^{\delta 1}$ and $\mathrm{H}^{n 2}$. The coupling factor ranges for these protons are highlighted (grey area) in figure 4.3. Furthermore, it is exemplary shown by vertical lines, how the ranges for distance of closest approach are obtained for the translation diffusion model. All calculated distances are given in table 4.6 alongside the calculated autorelaxation rates.

If one compares the obtained values of table 4.6 in regard to the contribution of inner sphere relaxation by the temporal average number $n$ of spins $\vec{I}$ inside the inner sphere, it becomes obvious that, the calculated distances $d$ increase with larger $n$ for all nuclei, but the calculated autorelaxation rate decreases in the same way. 
Table 4.6.: Calculated range of the distance of closest approach for three different protons of L-tryptophan $(10 \mathrm{mM})$ and TEMPONE- $\mathrm{d}_{16},{ }^{15} \mathrm{~N}$ in $\mathrm{D}_{2} \mathrm{O}$ at $42{ }^{\circ} \mathrm{C}$ based on the measured range of the coupling factor. The calculated autorelaxation rate of the translational diffusion model $(n=0)$ comes closest to the measured value. For the combined model of rotational and translational diffusion, the autorelaxation rate decreases and distance of closest approach increases from $n=0.0001$ to $n=0.01$.

\begin{tabular}{ccccccc}
\hline & $\mathrm{H}^{\alpha}$ & \multicolumn{3}{c}{$\mathrm{H}^{\delta 1}$} & $\mathrm{H}^{2}$ & \\
calculated: & $\rho\left[\mathrm{s}^{-1}\right]$ & $d[\AA]$ & $\rho\left[\mathrm{s}^{-1}\right]$ & $d[\AA]$ & $\rho\left[\mathrm{s}^{-1}\right]$ & $d[\AA]$ \\
\hline \hline$n=0$ & $1.13-1.19$ & $4.8-5.0$ & $1.49-1.66$ & $3.9-4.2$ & $1.95-2.46$ & $3.1-3.5$ \\
$n=0.0001$ & $1.12-1.18$ & $4.8-5.0$ & $1.47-1.63$ & $4.0-4.2$ & $1.92-2.42$ & $3.2-3.6$ \\
$n=0.001$ & $1.06-1.12$ & $5.2-5.4$ & $1.38-1.53$ & $4.4-4.6$ & $1.80-2.25$ & $3.7-4.1$ \\
$n=0.01$ & $0.87-0.91$ & $6.6-6.7$ & $1.12-1.24$ & $5.8-6.0$ & $1.45-1.83$ & $5.1-5.5$ \\
& & & & & & \\
\hline measured: & $\rho\left[\mathrm{s}^{-1}\right]$ & $\xi$ & $\rho\left[\mathrm{s}^{-1}\right]$ & $\xi$ & $\rho\left[\mathrm{s}^{-1}\right]$ & $\xi$ \\
\hline \hline & 2.18 & $0.13-0.14$ & 3.38 & $0.18-0.20$ & 3.56 & $0.23-0.27$ \\
\hline
\end{tabular}

Overall the calculated autorelaxation rates are at least by a factor of two too small in comparison to the experimental data. Nevertheless, these calculated rates still represent a very good result, if all assumptions, approximations and possible sources of experimental error are taken into account. The best values in regard to the experimental data were obtained with the model of pure translational diffusion ( $n=0$ - no inner sphere contribution), which is the first indication for translational diffusion model.

In addition, Sezer $\underline{[86]}$ reported a distance of closest approach of $3.0 \AA$ between the aromatic protons of the solvent toluene and the unpaired electron of 4-hydroxy2,2,6,6-tetramethyl-1-piperidinyloxyl (TEMPOL) at $300 \mathrm{~K}$. This distance was determined by molecular dynamic simulations in comparison with DNP measurements. The accessibility of aromatic toluene protons and the $\mathrm{H}^{\eta^{2}}$ proton should be almost identical. The same is expected from the nitroxyl-groups of TEMPONE- $\mathrm{T}_{16}{ }^{15} \mathrm{~N}$ and TEMPOL. Therefore, Sezer's distance should be directly transferable to the $\mathrm{H}^{n 2}$. For this proton, the distance range of closest approach obtained by the translational diffusion model fits best. This range is with $3.1 \AA$ to $3.5 \AA$ slightly longer than the reported $3.0 \AA$ which could be contributed to the different experimental condition and/or the experimental error. This finding is the second indication in favor of the translational diffusion model. 
All in all, the translational diffusion model is sufficient to give a qualitative description in regard to the experimental data of the spin-spin interaction between two dissolved spin bearing molecules at low fields and is in good agreement with results reported in the literature $\underline{[86]}$. The contribution by the inner sphere relaxation in the combined model is negligible in this context. The distance variation between different protons is between $0.4 \AA$ and $1.9 \AA$. These values are reasonable in relation to the distance of closest approach itself. Therefore, the calculations, here presented, support the hypothesis (see subsection 4.3.1), that the structure of a molecule directly and measurably influences the coupling factor. 


\subsection{DNP signal enhancement of carbon-13}

After the successful measurements of enhanced proton spectra, the DNP experiments were continued for ${ }^{13} \mathrm{C}$. The aim was to investigate, if even higher enhancements than for protons could be achieved for carbon corresponding to the difference between their respective gyromagnetic ratio. A theoretical maximum enhancement up to -31.6 is possible for pure dipolar interactions between the spins.

Furthermore, the translational diffusion model was applied to the carbons of L-tryptophan based on the results from section 4.3. Subsequently, the calculated ideal enhancements were compared with the experimental results. In addition, it was investigated in a concluding step, how the presence of a strongly coupled spin, for example a proton, changes the carbon enhancements. This step serves as a preparation for measurements of ${ }^{13} \mathrm{C}$ or ${ }^{15} \mathrm{~N}$ labeled biomacromolecules.

\subsubsection{Carbon enhancement in a two-spin system}

The measured hf ${ }^{13} \mathrm{C}$ enhancements with deuterated ${ }^{13} \mathrm{C}$ and ${ }^{15} \mathrm{~N}$ labeled L-tryptophan were much smaller than expected from the theoretical maximum enhancement of -31.6 . These ${ }^{13} \mathrm{C}$ enhancements ranged from -0.3 to -2.5 as illustrated in figure 4.4, For a better understanding of this result, the range of the carbon coupling factor was calculated. This range was based on the translational diffusion model and the calculated ranges of the distance of closest approach for the example protons of L-tryptophan. The procedure was identical to subsection 4.3 .2 but it was assumed, that the distances of closest approach for carbons is increased due to the bonded example protons which reduce the accessibility of the carbon nuclei. Therefore, the calculated distances were extended by the proton-carbon bond length of $1.1 \AA$. Furthermore, an estimation of the theoretical maximum high-field enhancement was calculated with the calculated range of the coupling factor. For this purpose, an ideal leakage and saturation factor of 1 was assumed in addition to zero relaxation losses during the sample transfer. The obtained theoretical maximum enhancements $\varepsilon_{\mathrm{hf} \text { max }}$ alongside the calculated coupling factors are given in table 4.7 for the carbons $\mathrm{C}^{\alpha}, \mathrm{C}^{\delta 1}$ and $\mathrm{C}^{n 2}$. These are bonded to the previously (subsection 4.3.2) selected example protons. 
Table 4.7.: Calculated enhancements for L-tryptophan- $\mathrm{d}_{8},{ }^{15} \mathrm{~N}_{2},{ }^{13} \mathrm{C}_{11}$ and corresponding parameters of the translational diffusion model

\begin{tabular}{cccc}
\hline & $\mathrm{C}^{\alpha}$ & $\mathrm{C}^{\delta 1}$ & $\mathrm{C}^{\eta^{2}}$ \\
\hline \hline measured: & -0.3 & -2.1 & -1.8 \\
$\varepsilon_{\mathrm{hf}}$ & & & \\
& & & \\
calculated: & $4.9-6.1$ & $5.0-5.3$ & $4.2-4.6$ \\
$d[\AA]$ & $0.08-0.09$ & $0.11-0.13$ & $0.15-0.18$ \\
$\xi$ & $-5.0--5.4$ & $-7.1--7.9$ & $-9.3--11.2$ \\
$\varepsilon_{\mathrm{hf}, \max }$ & &
\end{tabular}

The calculated coupling factors are between 0.08 and 0.18 and therefore, result in a theoretical maximum enhancements $\varepsilon_{\mathrm{hf}, \max }$ of -5 to -11 . Of course, these ideal enhancements are reduced by different influences under the experimental conditions. So far, a leakage factor of 1 was assumed, but a smaller leakage factor is more likely, if one takes the previously measured leakage factors of the protons of L-tryptophan into account. These have an average value of about 0.8 .

Additionally, one has to consider, that the interactions between the carbons and the unpaired electron of the radical are weaker than for the protons due to the longer

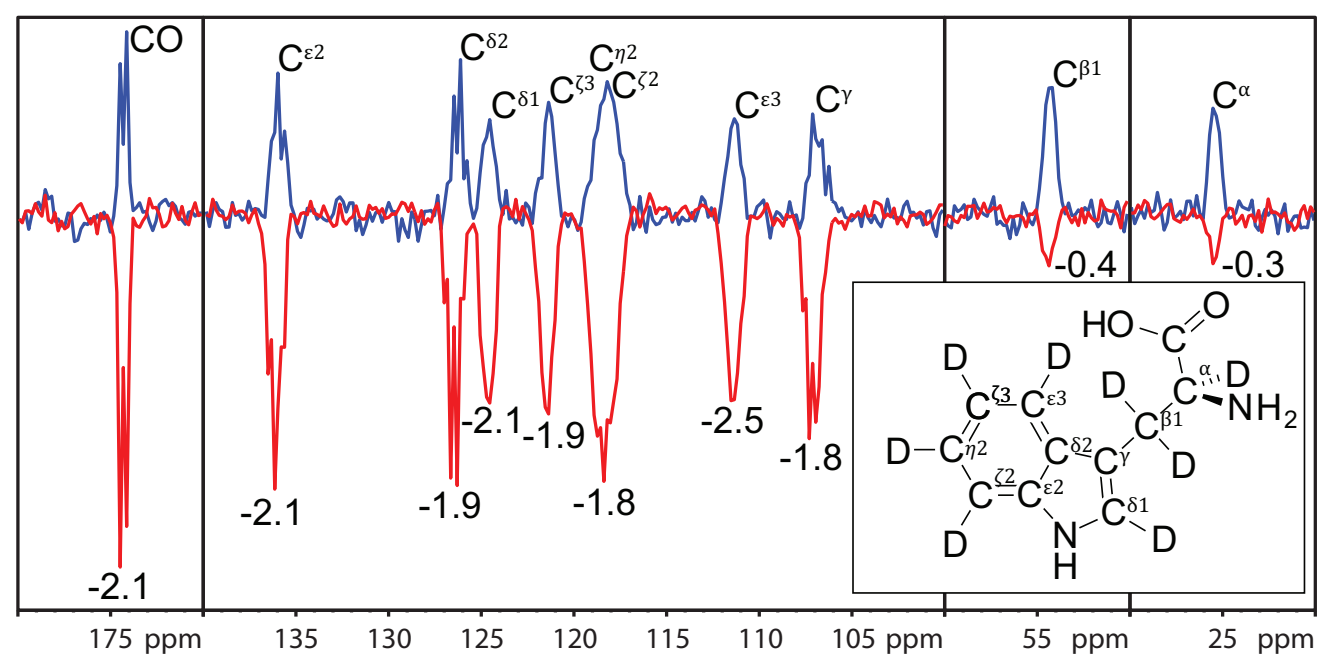

Figure 4.4.: ${ }^{13} \mathrm{C}$ enhancements of L-tryptophan- $\mathrm{d}_{8},{ }^{15} \mathrm{~N}_{2},{ }^{13} \mathrm{C}_{11}(50 \mathrm{mM})$ in $\mathrm{D}_{2} \mathrm{O}$ with TEMPONE- $\mathrm{d}_{16},{ }^{15} \mathrm{~N}(10 \mathrm{mM})$. The experimental parameters were $t_{\mathrm{p}}=10 \mathrm{~s}, P_{\mathrm{MW}}=6.7 \mathrm{~W}$ and 4096 scans 
distance of closest approach and due to the smaller gyromagnetic ratio. Furthermore, there is the strong dipolar interaction between neighboring carbon nuclei which represent additional relaxation pathways for "leakage". Altogether, these aspects lead to an estimated leakage factor of about 0.5 .

Likewise, the saturation factor is smaller than 1 under the experimental conditions. A saturation factor of $s=0.85$ was assumed for the proton experiments. For the carbon experiments, however, one must assume an even smaller saturation factor due to a very long $\mathrm{mw}$ irradiation time $\left(t_{\mathrm{p}}=10 \mathrm{~s}\right)$. Despite the active cooling, the DNP cavity gradually heats up during that time and is no longer fully matched. The consequence is a reduced $\mathrm{mw}$ power input and thereby, an only partially saturated EPR line. This results in a smaller saturation factor about 0.6 to 0.7.

In addition to the reduced leakage and saturation factor, relaxation losses occur during the sample transfer from the low-field to the high-field. These are presumably about 10 to $20 \%$. Taking all these effects into account, the calculated theoretical maximum carbon enhancement is reduced to approximately one fourth which is close to the measured enhancements.

In conclusion, the magnitude of the measured enhancements of carbon are plausible and the translational diffusion model is useful for a qualitative estimation of the achievable enhancements.

\subsubsection{Carbon enhancement in a three-spin system}

As a preliminary step to protein measurements, carbon DNP experiments were conducted on protonated, ${ }^{13} \mathrm{C}$ - and ${ }^{15} \mathrm{~N}$-labeled L-tryptophan since most biomacromolecules are labeled with ${ }^{13} \mathrm{C}$ and/or ${ }^{15} \mathrm{~N}$ with the purpose to increase the sensitivity in hetero nuclear correlation experiments. With three different spins present, the DNP transfer becomes more complex. The strong dipolar interaction between the proton and the carbon could lead to additional polarization transfer between these nuclei. The result would be a positive enhancement for the carbon nuclei. This so called "three-spin-effect" was described and investigated by Natusch et al. ${ }^{[87]}$. So far, it has been only observed for solvents ${ }^{[87,88]}$. Here, the effect was observed for the first time for solute molecules. The measured carbon enhancements for L-tryptophan${ }^{15} \mathrm{~N}_{2},{ }^{13} \mathrm{C}_{11}$ are all positive and between 1.0 and 2.3 , except for the carbonyl carbon 


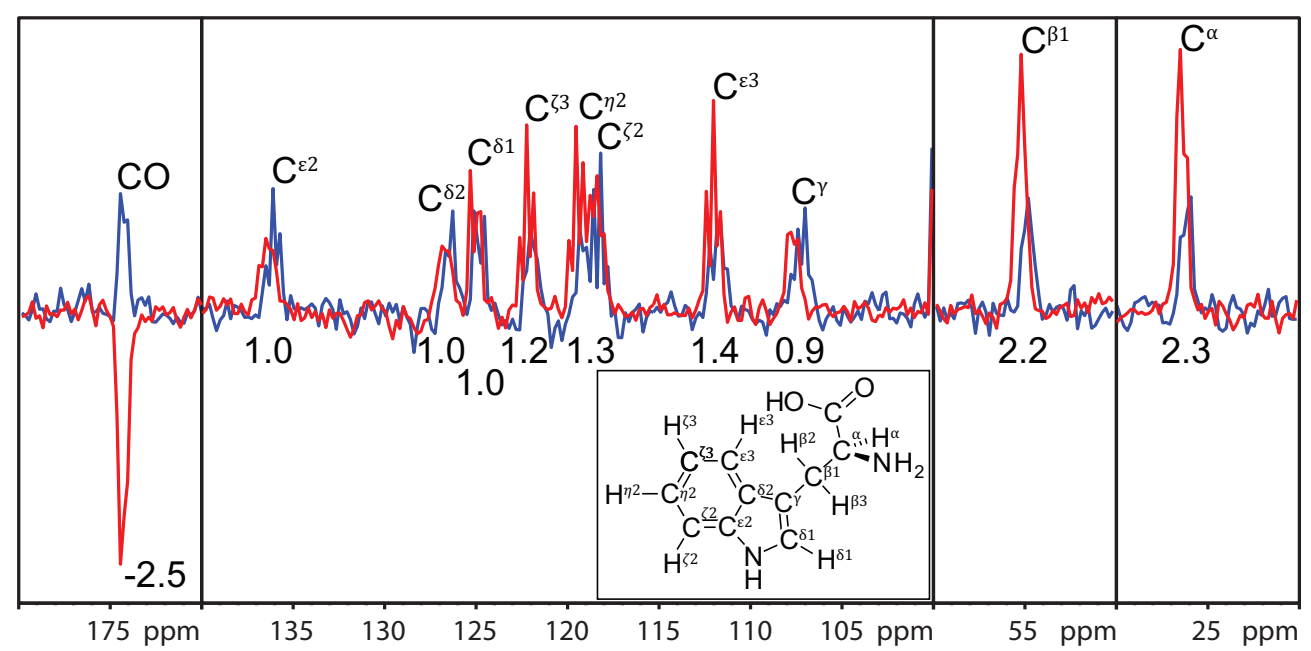

Figure 4.5.: ${ }^{13} \mathrm{C}$ enhancements of L-tryptophan $-{ }^{15} \mathrm{~N}_{2},{ }^{13} \mathrm{C}_{11}(50 \mathrm{mM})$ in $\mathrm{D}_{2} \mathrm{O}$ with ${\text { TEMPONE- } \mathrm{d}_{16},{ }^{15} \mathrm{~N}}(10 \mathrm{mM})$. The experimental parameters were $t_{\mathrm{p}}=10 \mathrm{~s}, P_{\mathrm{MW}}=6.7 \mathrm{~W}$ and 4096 scans

with an negative enhancement of -2.5 (figure 4.5).

For a theoretical treatment of these results, it is necessary to expand the coupling factor to the dipolar interaction in the three spin system. These interactions are described by the following relaxation matrix:

$$
\left(\begin{array}{c}
\dot{H}_{\mathrm{z}} \\
\dot{C}_{\mathrm{z}} \\
\dot{e}_{\mathrm{z}}
\end{array}\right)=-\left(\begin{array}{ccc}
\rho_{\mathrm{H}} & \sigma_{\mathrm{HC}} & \sigma_{\mathrm{He}} \\
\sigma_{\mathrm{HC}} & \rho_{\mathrm{C}} & \sigma_{\mathrm{Ce}} \\
\sigma_{\mathrm{He}} & \sigma_{\mathrm{Ce}} & \rho_{\mathrm{e}}
\end{array}\right)\left(\begin{array}{c}
H_{\mathrm{z}}-H_{0} \\
C_{\mathrm{z}}-C_{0} \\
e_{\mathrm{z}}-e_{0}
\end{array}\right)
$$

$H_{\mathrm{z}}, C_{\mathrm{z}}$ and $e_{\mathrm{z}}$ are the polarizations of the protons, carbons and electrons along the z-axis. The additional index 0 indicates the thermal equilibrium polarization along the z-axis. Furthermore, $\sigma_{\mathrm{He}}, \sigma_{\mathrm{Ce}}$ and $\sigma_{\mathrm{HC}}$ are the crossrelaxation rates of the proton-electron interaction, the carbon-electron interaction and the proton-carbon interaction, respectively (see equation 1.14). Here $\rho$ represents the autorelaxation rate of each respective spin. This rate includes all transition probabilities with the other two spins analogous to figure 1.1 and equation 1.14:

$$
\rho_{\mathrm{H}}=w_{2, \mathrm{He}}+2 w_{1, \mathrm{He}}+w_{0, \mathrm{He}}+w_{2, \mathrm{HC}}+2 w_{1, \mathrm{HC}}+w_{0, \mathrm{HC}}
$$




$$
\begin{gathered}
\rho_{\mathrm{C}}=w_{2, \mathrm{Ce}}+2 w_{1, \mathrm{Ce}}+w_{0, \mathrm{Ce}}+w_{2, \mathrm{HC}}+2 w_{1, \mathrm{HC}}^{\prime}+w_{0, \mathrm{HC}} \\
\rho_{\mathrm{e}}=w_{2, \mathrm{He}}+2 w_{1, \mathrm{He}}^{\prime}+w_{0, \mathrm{He}}+w_{2, \mathrm{Ce}}+2 w_{1, \mathrm{Ce}}^{\prime}+w_{0, \mathrm{Ce}}
\end{gathered}
$$

For the DNP experiment, it is assumed, that the polarization of the spins reached a steady state condition which gives for equation 4.21:

$$
\left(\begin{array}{c}
0 \\
0 \\
\dot{e}_{\mathrm{z}}
\end{array}\right)=-\left(\begin{array}{ccc}
\rho_{\mathrm{H}} & \sigma_{\mathrm{HC}} & \sigma_{\mathrm{He}} \\
\sigma_{\mathrm{HC}} & \rho_{\mathrm{C}} & \sigma_{\mathrm{Ce}} \\
\sigma_{\mathrm{He}} & \sigma_{\mathrm{Ce}} & \rho_{\mathrm{e}}
\end{array}\right)\left(\begin{array}{c}
H_{\mathrm{z}}-H_{0} \\
C_{\mathrm{z}}-C_{0} \\
e_{\mathrm{z}}-e_{0}
\end{array}\right)
$$

This equation is separated into three single equations:

$$
\begin{gathered}
0=-\rho_{\mathrm{H}}\left(H_{\mathrm{z}}-H_{0}\right)-\sigma_{\mathrm{HC}}\left(C_{\mathrm{z}}-C_{0}\right)+\sigma_{\mathrm{He}}\left(e_{0}-e_{\mathrm{z}}\right) \\
0=-\sigma_{\mathrm{HC}}\left(H_{\mathrm{z}}-H_{0}\right)-\rho_{\mathrm{C}}\left(C_{\mathrm{z}}-C_{0}\right)+\sigma_{\mathrm{Ce}}\left(e_{0}-e_{\mathrm{z}}\right) \\
\dot{e}_{\mathrm{z}}=-\sigma_{\mathrm{He}}\left(H_{\mathrm{z}}-H_{0}\right)-\sigma_{\mathrm{Ce}}\left(C_{\mathrm{z}}-C_{0}\right)+\rho_{\mathrm{e}}\left(e_{0}-e_{\mathrm{z}}\right)
\end{gathered}
$$

Since only the nuclear polarization is of interest, equations 4.26 and 4.27 are recombined:

$$
\left(\begin{array}{c}
\sigma_{\mathrm{He}} \\
\sigma_{\mathrm{Ce}}
\end{array}\right)\left(e_{0}-e_{\mathrm{z}}\right)=\left(\begin{array}{cc}
\rho_{\mathrm{H}} & \sigma_{\mathrm{HC}} \\
\sigma_{\mathrm{HC}} & \rho_{\mathrm{C}}
\end{array}\right)\left(\begin{array}{c}
H_{\mathrm{z}}-H_{0} \\
C_{\mathrm{z}}-C_{0}
\end{array}\right)
$$

and rearranged:

$$
\left(\begin{array}{cc}
\rho_{\mathrm{C}} & -\sigma_{\mathrm{HC}} \\
-\sigma_{\mathrm{HC}} & \rho_{\mathrm{H}}
\end{array}\right)\left(\rho_{\mathrm{H}} \rho_{\mathrm{C}}-\sigma_{\mathrm{HC}}^{2}\right)^{-1}\left(\begin{array}{c}
\sigma_{\mathrm{He}} \\
\sigma_{\mathrm{Ce}}
\end{array}\right)\left(e_{0}-e_{\mathrm{z}}\right)=\left(\begin{array}{c}
H_{\mathrm{z}}-H_{0} \\
C_{\mathrm{z}}-C_{0}
\end{array}\right)
$$

to give the polarizations of protons and carbons after a second separation:

$$
\begin{aligned}
& H_{\mathrm{z}}-H_{0}=\frac{\sigma_{\mathrm{He}} \rho_{\mathrm{C}}-\sigma_{\mathrm{Ce}} \sigma_{\mathrm{HC}}}{\rho_{\mathrm{H}} \rho_{\mathrm{C}}-\sigma_{\mathrm{HC}}^{2}}\left(e_{0}-e_{\mathrm{z}}\right) \\
& C_{\mathrm{z}}-C_{0}=\frac{\sigma_{\mathrm{Ce}} \rho_{\mathrm{H}}-\sigma_{\mathrm{He}} \sigma_{\mathrm{HC}}}{\rho_{\mathrm{H}} \rho_{\mathrm{C}}-\sigma_{\mathrm{HC}}^{2}}\left(e_{0}-e_{\mathrm{z}}\right)
\end{aligned}
$$


According to subsection 1.3.1, these two equations are expanded by $\left(\frac{e_{0}}{e_{0} H_{0}}\right)$ and $\left(\frac{e_{0}}{e_{0} C_{0}}\right)$, respectively:

$$
\begin{gathered}
\frac{H_{\mathrm{z}}-H_{0}}{H_{0}}=\left(\frac{\sigma_{\mathrm{He}} \rho_{\mathrm{C}}-\sigma_{\mathrm{Ce}} \sigma_{\mathrm{HC}}}{\rho_{\mathrm{H}} \rho_{\mathrm{C}}-\sigma_{\mathrm{HC}}^{2}}\right)\left(\frac{e_{0}-e_{\mathrm{z}}}{e_{0}}\right)\left(\frac{e_{0}}{H_{0}}\right) \\
\frac{C_{\mathrm{z}}-C_{0}}{C_{0}}=\left(\frac{\sigma_{\mathrm{Ce}} \rho_{\mathrm{H}}-\sigma_{\mathrm{He}} \sigma_{\mathrm{HC}}}{\rho_{\mathrm{H}} \rho_{\mathrm{C}}-\sigma_{\mathrm{HC}}^{2}}\right)\left(\frac{e_{0}-e_{\mathrm{z}}}{e_{0}}\right)\left(\frac{e_{0}}{C_{0}}\right)
\end{gathered}
$$

By inserting each of these equations in the equation for the enhancement factor 1.17 and using the equation of the saturation factor 1.21, one ends up with the signal enhancement in a three spin system without leakage:

$$
\begin{gathered}
\varepsilon_{\mathrm{HC}, \mathrm{H}}=1+\left(\frac{\sigma_{\mathrm{He}} \rho_{\mathrm{C}}-\sigma_{\mathrm{Ce}} \sigma_{\mathrm{HC}}}{\rho_{\mathrm{H}} \rho_{\mathrm{C}}-\sigma_{\mathrm{HC}}^{2}}\right) s\left(\frac{\gamma_{\mathrm{e}}}{\gamma_{\mathrm{H}}}\right) \\
\varepsilon_{\mathrm{HC}, \mathrm{C}}=1+\left(\frac{\sigma_{\mathrm{Ce}} \rho_{\mathrm{H}}-\sigma_{\mathrm{He}} \sigma_{\mathrm{HC}}}{\rho_{\mathrm{H}} \rho_{\mathrm{C}}-\sigma_{\mathrm{HC}}^{2}}\right) s\left(\frac{\gamma_{\mathrm{e}}}{\gamma_{\mathrm{C}}}\right)
\end{gathered}
$$

As in equation 1.23, the thermal equilibrium polarizations $H_{0}, C_{0}$ and $e_{0}$ were substituted by the corresponding gyromagnetic ratios. For the comparison with the experimental results, the high-field enhancement is obtained by adding the field ratio to equation 4.36:

$$
\varepsilon_{\mathrm{hf}, \mathrm{HC}, \mathrm{C}}=\left[1+\left(\frac{\sigma_{\mathrm{Ce}} \rho_{\mathrm{H}}-\sigma_{\mathrm{He}} \sigma_{\mathrm{HC}}}{\rho_{\mathrm{H}} \rho_{\mathrm{C}}-\sigma_{\mathrm{HC}}^{2}}\right) s\left(\frac{\gamma_{\mathrm{e}}}{\gamma_{\mathrm{C}}}\right)\right]\left(\frac{B_{0, \mathrm{lf}}}{B_{0, \mathrm{hf}}}\right)
$$

The first fraction of equation 4.36 corresponds to the coupling factor of carbon with an electron and a proton in the three spin system

$$
\xi_{\mathrm{HC}, \mathrm{C}}=\left(\frac{\sigma_{\mathrm{Ce}} \rho_{\mathrm{H}}-\sigma_{\mathrm{He}} \sigma_{\mathrm{HC}}}{\rho_{\mathrm{H}} \rho_{\mathrm{C}}-\sigma_{\mathrm{HC}}^{2}}\right)
$$

For the intermolecular dipolar electron-proton and electron-carbon interaction the translational diffusion model was used analogously to the previous subsection 4.4.2. The intramolecular dipolar interaction between proton and carbon was calculated with the model of molecular tumbling based on the equation for the spectral density 
Table 4.8.: Calculated enhancements for L-tryptophan- ${ }^{15} \mathrm{~N}_{2},{ }^{13} \mathrm{C}_{11}$ and the corresponding parameters of the translational diffusion model

\begin{tabular}{cccc}
\hline & $\mathrm{C}^{\alpha}$ & $\mathrm{C}^{\tilde{1}}$ & $\mathrm{C}^{\eta^{2}}$ \\
\hline \hline measured: & 2.3 & 1.0 & 1.3 \\
$\varepsilon_{\mathrm{hf}}$ & & & \\
& & & \\
calculated: & $4.9-6.1$ & $5.0-5.3$ & $4.2-4.6$ \\
$d[\AA]$ & $-0.03--0.04$ & $-0.04--0.05$ & $-0.05--0.06$ \\
$\xi$ & $2.2-2.3$ & $2.9-3.1$ & $3.4-3.6$ \\
$\varepsilon_{\mathrm{hf}, \max }$ & & & \\
\hline
\end{tabular}

1.35 and the equation for the proportionality factor 1.36. The distance between proton and carbon corresponds to their bond length of $1.1 \AA$.

With these equations, the coupling factors and the theoretical maximum enhancements for the three example carbons from subsection 4.4.1 were calculated and summarized in table 4.8 .

The calculated coupling factors are negative. Hence, the enhancements are positive for all three example carbons. Therefore the sign of the enhancement is in agreement with the experimental findings. Although, the magnitude of the ideal calculated enhancements are too small, if one applies the same considerations about the experimental conditions as in subsection 4.4.1. A the leakage factor of $f=0.5$, a the saturation factor of $s=0.7$ and relaxation losses of $10 \%$ to $20 \%$ result in enhancements below the measured values, especially for the $\mathrm{H}^{\alpha}$.

In conclusion, the positive enhancements are qualitatively explained by including the proton-carbon interaction, but quantitative predictions are not possible. The used approach is with only three considered spins to superficial. One indication are the positive enhanced quaternary carbons which were not taken into account so far. They presumably interact with all three protons bound to their neighboring atoms, from where the polarization is transferred. A single proton is not efficient enough for this long distance interaction since the carbonyl carbon with one proton in close proximity shows a negative enhancement. For more accurate predictions of the coupling factor, it would be necessary to include all spins of the molecule. 


\subsection{First results on ubiquitin}

After the preparatory measurements on ${ }^{13} \mathrm{C}$-labeled and ${ }^{15} \mathrm{C}$-labeled L-tryptophan, DNP experiments on a small fully labeled biomacromolecule were conducted to investigated if it is possible to transfer polarization to the surface of a protein. For this purpose, Ubiquitin-U- ${ }^{15} \mathrm{~N}, \mathrm{U}-{ }^{13} \mathrm{C}$ was chosen as a small globular protein with a molecular mass of $8.5 \mathrm{kDa}$. It is stable under various conditions and a well studied protein $[73,89,90]$.

The dependency of the proton coupling factor of ubiquitin on the distance of closest approach was calculated for the case of a three-spin system in regard to the results of L-tryptophan. It includes the interaction between the proton and carbon spin. Additionally, the two-spin case without carbon spins was calculated to investigate the influence of proton-carbon interaction.

The procedure of the calculations was identical to L-tryptophan (section 4.3 and 4.4). The translational diffusion model was applied with the diffusion constant of ubiquitin reported by Mayo et al. [91]. The intramolecular proton-carbon interaction was again based on the molecular tumbling with the rotational correlation time of ubiquitin reported by Tjandra et al. ${ }^{[92]}$. As done before, both literature values were

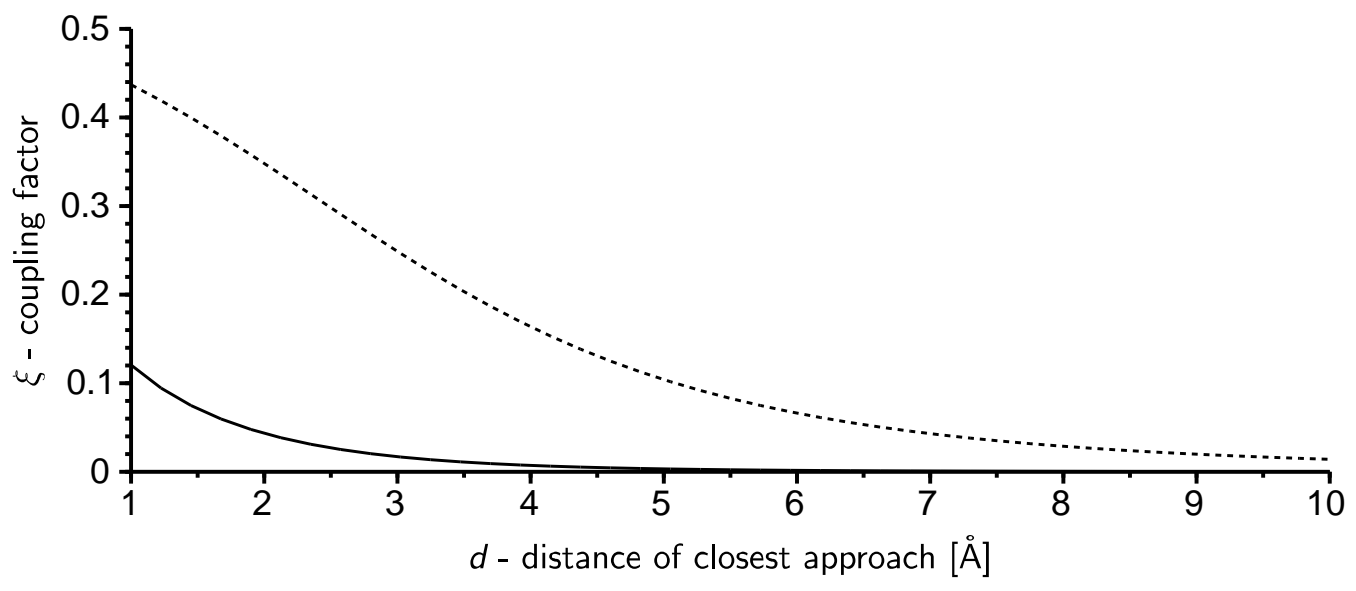

Figure 4.6.: Dependency of the proton coupling factor on the distance of closest approach for Ubiquitin-U- ${ }^{15} \mathrm{~N}, \mathrm{U}-{ }^{13} \mathrm{C}$ and TEMPONE- $\mathrm{d}_{16},{ }^{15} \mathrm{~N}$ in $\mathrm{D}_{2} \mathrm{O}$ at $54{ }^{\circ} \mathrm{C}$. The solid line represents the translational diffusion model applied to a three-spin system of a proton, a carbon and an electron. The dotted line corresponds to a two-spin system of a proton and an electron. 
scaled with the equations 4.19 and 4.20 to the experimental conditions of $54{ }^{\circ} \mathrm{C}$ and $\mathrm{D}_{2} \mathrm{O}$ as solvent. The result for the three- (solid line) and two-spin (dotted line) system is shown in figure 4.6.

The obtainable coupling factor relative to the distance of the closest approach for the three-spin system is significantly smaller than for the two-spin system. The long rotational correlation time of ubiquitin causes a strong proton-carbon interaction, which diminishes the overall coupling factor. Therefore only a weak polarization transfer is expected from the electron to the surface of the protein. Due to this, the experimental set-up is changed for a clear distinction between the nuclei, which are effected by polarization transfer from the electron, and the nuclei, which are only effected by the sample transfer to the low field. For this reason, the DNP experiment was compared to an identically experiment with identical delays and durations but without microwave irradiation. By this means, even small changes in the signal intensity due to a polarization transfer could be identified.

In a preliminary step before conducting the DNP experiment, a spectrum with Boltzmann polarization was recorded in the high-field position. The two dimensional ${ }^{1} \mathrm{H}-{ }^{13} \mathrm{C}-$ Heteronuclear Single Quantum Coherence (HSQC) spectrum of the protein sample with only $23 \mathrm{nmol}(5 \mathrm{mM})$ Ubiquitin-U- ${ }^{15} \mathrm{~N}, \mathrm{U}-{ }^{13} \mathrm{C}$ and $10 \mathrm{mM}$ TEMPONE- $\mathrm{d}_{16},{ }^{15} \mathrm{~N}$ in $\mathrm{D}_{2} \mathrm{O}$ is depicted in figure 4.7 (A) with a close up of the methyl region $(\mathrm{B})$. The resolution and the sensitivity is sufficient to identify single resonances. The subsequently recorded DNP experiment and the corresponding shuttle experiment showed for most resonances no significant differences within the experimental error. The main reason are the relaxation losses during the sample transfer in addition to the predicted weak polarization transfer. Nevertheless, it was possible to extract two important information from the recorded spectra.

Firstly, a few signals show a distinct reduction of their intensity in the DNP experiment compared to the identical shuttle experiment without $\mathrm{mw}$ irradiation. The most prominent examples are illustrated in figure 4.8. The assignment of the ubiquitin ${ }^{[89]}$ resonances was taken from the Biological Magnetic Resonance Data Bank (BRMB ${ }^{[90]}$. The signals of the lysine $63 \mathrm{H}^{\varepsilon}$ protons and of the arginine $74 \mathrm{H}^{\delta}$ protons are highlighted in figure 4.8. Both signals are well resolved and distinct from other signals in the shuttle experiment without $\mathrm{mw}$ irradiation (left). By contrast, the two signals are nearly gone compared to the other signals in the 

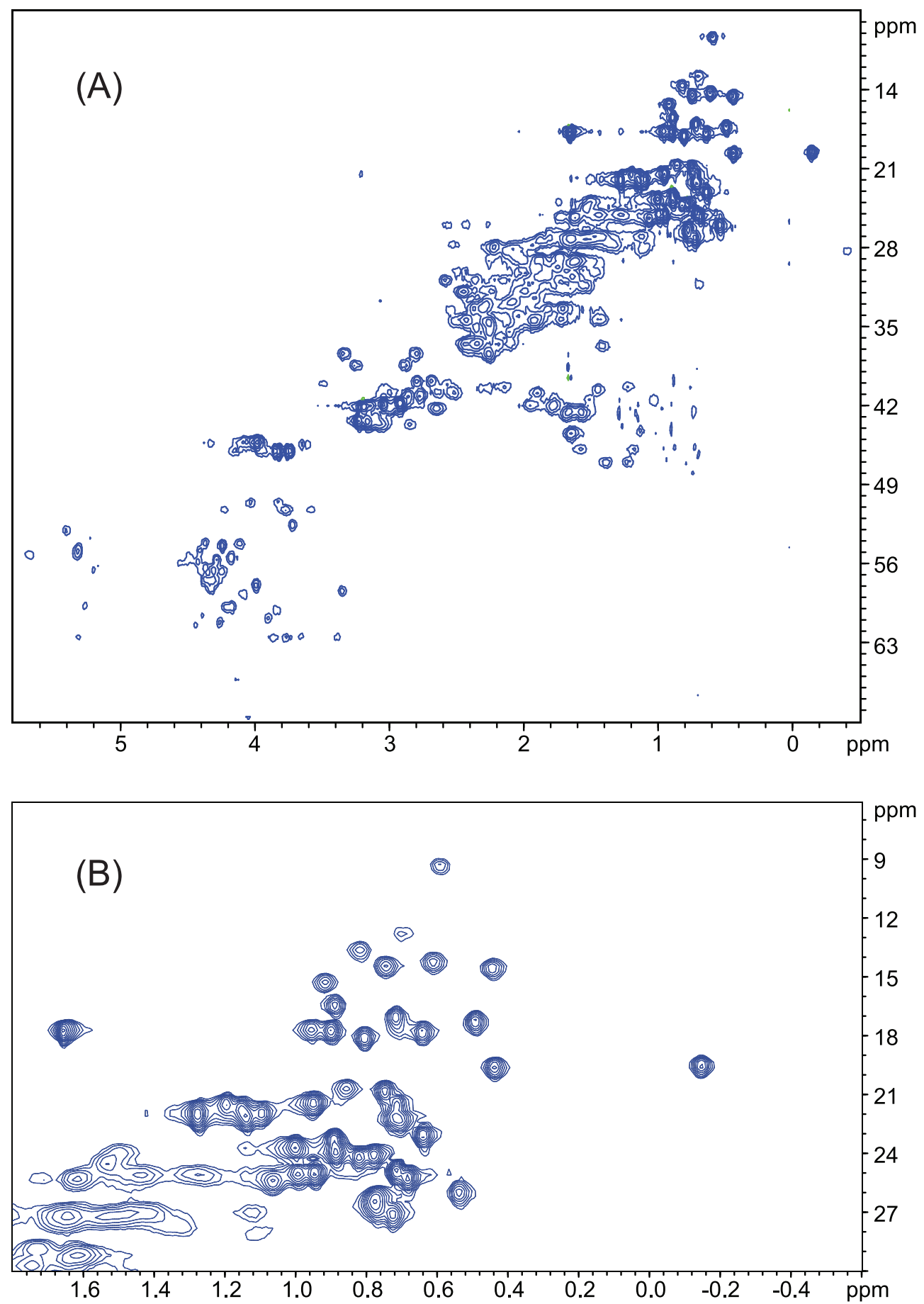

Figure 4.7.: ${ }^{1} \mathrm{H}_{-}{ }^{13} \mathrm{C}-\mathrm{HSQC}$ spectrum of $23 \mathrm{nmol}(5 \mathrm{mM})$ Ubiquitin-U- ${ }^{15} \mathrm{~N}, \mathrm{U}_{-}{ }^{13} \mathrm{C}$ with $10 \mathrm{mM}$ TEMPONE- $\mathrm{d}_{16}{ }^{15} \mathrm{~N}, 20 \mathrm{mM}$ DSS and $50 \mathrm{mM} \mathrm{NaCl}$ in $50 \mathrm{mM}$ phosphate buffer with $\mathrm{D}_{2} \mathrm{O}$ as solvent - (A) full spectrum (B) methyl region 

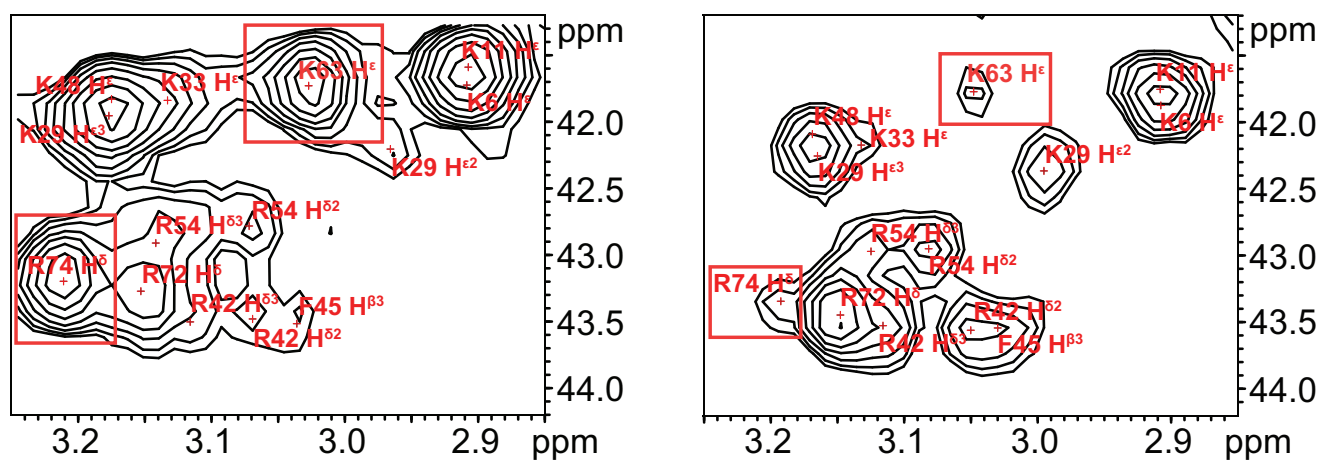

Figure 4.8.: Shuttle (left) and DNP (right) ${ }^{1} \mathrm{H}_{-}{ }^{13} \mathrm{C}-\mathrm{HSQC}$ spectrum of $23 \mathrm{nmol}(5 \mathrm{mM})$ Ubiquitin-U- ${ }^{15} \mathrm{~N}, \mathrm{U}-{ }^{13} \mathrm{C}$ with $10 \mathrm{mM}$ TEMPONE- $\mathrm{d}_{16},{ }^{15} \mathrm{~N}, 20 \mathrm{mM}$ DSS and $50 \mathrm{mM} \mathrm{NaCl}$ in $50 \mathrm{mM}$ phosphate buffer with $\mathrm{D}_{2} \mathrm{O}$ as solvent $-t_{\mathrm{p}}$ was $2 \mathrm{~s}$ and $P_{\mathrm{MW}}$ was $11 \mathrm{~W}$

DNP experiment with $\mathrm{mw}$ irradiation. Even though, the other depicted signals show intensity changes, these changes are not as clear as the reduction of the lysine 63 and arginine 74 signals.

Since the only difference between the two conducted experiments is the irradiation with the microwave, the intensity change must be caused by it. Altogether, there are two possible explanations which are based on the relaxation during the sample transfer.

One of these two explanation is, that the microwave irradiation heats up the protein sample. Thereby, the molecular tumbling and the diffusion rates increase with the consequence, that the longitudinal relaxation times of the protons increase. These longer relaxation times would slow down the build-up of the high-field polarization back to thermal equilibrium during the transfer of the sample from the low-field to the high-field position. The result would be a smaller signal intensity compared to the shuttle experiment without $\mathrm{mw}$ heating. However, this change effects all signals of ubiquitin by a similar degree and not only individual signals, such as lysine 63 and arginine 74 .

The other explanation is, that polarization was transferred from the electron to the mentioned protons resulting in an negative enhancement. However, the negative polarization relaxed back through null to a weak positive polarization during the transfer. This polarization is still smaller than the polarization of the shuttle experiment without $\mathrm{mw}$ irradiation. Hence, the conclusion is, that for the first time 
in liquid state $\mathrm{DNP}$, protons of a protein were successfully polarized by the method of DNP.

The second extracted information is based on the previous conclusion. The polarization transfer is especially observed on the accessible protons of the side chains on the surface of the protein. The implication is, that surface information of a protein are obtainable by solely observing the polarization transfer from the electron to exposed protons. Furthermore this accessibility dependence is another confirmation of the translational diffusion model and the importance of the distance of closest approach for the polarization transfer. 


\subsection{Outlook}

In this thesis, the parameters of protons, which are relevant for shuttle DNP, have been elaborately studied for small molecules. For all of these parameters (coupling and leakage factor as well as the relaxation losses during the transfer), data were collected and theoretical models were established. This has been different for carbons of small molecules, where further measurements are necessary. Additional relaxation measurements would enable the calculation of the leakage factor and the relaxation losses. With these two parameters, it would be possible to calculate the coupling factor of the carbon nuclei based on measured data and to calculate in a subsequent step the distance of closest approach with the translational diffusion model. These distances would help to determine, if the translational diffusion model could be used for qualitative predictions of the coupling factor.

For those additional measurements, the experimental condition could be improved further. Higher radical concentrations $(>10 \mathrm{mM})$ increase the leakage factor and reduce the build-up time of the carbon nuclei. The consequences are shorter $\mathrm{mw}$ irradiation times, more time effective measurements and less heating of the DNP cavity.

The future efforts to polarize proteins will be divided up into two different approaches. The first approach is to investigate proteins with a rotational correlation time of approximately 40 ns. For comparison, ubiquitin has a rotational correlation time of $4.1 \mathrm{~ns}{ }^{[92]}$. For slow tumbling proteins above a threshold of 12 ns, the dipolar relaxation between the protons of the protein is no longer destructive. This means, that the net magnetization of the protons is preserved at the magnetic field strength of $0.34 \mathrm{~T}$. This effect is called spin diffusion and enables the transfer of the dynamic nuclear polarization from the surface into the center of the protein.

Furthermore, a beneficial side effect for carbon labeled proteins with increasing rotational correlation times is a decreasing cross relaxation rate between proton and carbon spins, which interferes with the polarization transfer from the electron to the proton. Therefore, a reduced cross relaxation rate leads to a higher coupling factor of the protons in a three-spin system with a carbon and an electron.

The second approach will focus on the enhancements of amide protons in the back bone of a protein. These protons exchange with the water protons of the bulk 
solution. The rate of this chemical exchange depends on accessibility of the amide group by the solvent. Thereby, it would be possible to transfer protons from the surrounding water to the backbone amides which exhibit a fast exchange rate. Since enhancement factors of up to -4 are achievable for water, significant enhancements are expected for the exchanging amide protons, too. 
Appendices 



\section{A. Tables}

\section{A.1. List of abbreviations}

BCU Bruker Cooling Unit. 27

BRMB Biological Magnetic Resonance Data Bank. 71

DNP Dynamic Nuclear Polarization. III VI, 11, 3] 13, 16, 18, 22, 25, 27, 30, 32, 35, 37139, 43, 44, 46, 55, 61, 63, 65, 67, 70, 71, 73, 75, 82, 91, 94, 96, 97, 107

DOSY Diffusion ordered NMR spectroscopy. 39, 41

DSS sodium 2,2-Dimethyl-2-Silapentane-1-Sulfonate. [35, 37, 40, 43, 44, 51, 55, 72, 73,82

EPR Electron Paramagnetic Resonance. 21, 25, 27, 44, 65, 82

FID Free Induction Decay. 40, 48, 52, 53

hf High Field. 4, 6, 7, 21, 23, 30, 63,

HSQC Heteronuclear Single Quantum Coherence. IV, VI, 40, 41, 71773, 82, 100, 103, 107

INEPT Insensitive Nuclei Enhanced by Polarisation Transfer. 40

If Low Field. 6, 8, 21, 23, 27, 30, 43, 57,

MAS Magnetic Angle Spinning. 4, 7 
MRI Magnetic Resonance Imaging. 4, 5]

mw Microwave. 4, 5, 14, 26, 37, 38, 65, 71, 73, 175, 82

NMR Nuclear Magnetic Resonance. III, V, 117, 15, 21, 23, 25, 26, 29, 30, 32, 33, 38, 39, 41, 46, 48, 58, 82, 91

NMRD Nuclear Magnetic Relaxation Dispersion. 18

NOE Nuclear Overhauser Effect. 4

RF Radio Frequency. 32, 49

SNR Signal-to-Noise Ratio. 8, 46, 50, 52, 53

TEMPOL 4-hydroxyl-2,2,6,6-TEtraMethyl-1-PiperidinylOxyl-. 61

TEMPONE- $\mathbf{d}_{16},{ }^{15} \mathbf{N}$ 4-oxo-2,2,6,6-TEtraMethyl-1-PiperidinylOxyl- $d_{16},{ }^{15} \mathrm{~N}$. 19, 35, 39, 43, 44, 51, 55, 56, 58, 61, 64, 66, 70, 73, 81 


\section{A.2. List of Tables}

4.1. Measured signal enhancement of L-tryptophan . . . . . . . . . . . . . 44

4.2. Calculated global enhancement factor of L-tryptophan . . . . . . . . 47

4.3. Calculated applied global enhancement factor of $\mathrm{H}^{\delta 1}$ of L-tryptophan 51

4.4. Calculated coupling factors of L-tryptophan . . . . . . . . . . . 55

4.5. Calculated parameters of TEMPONE- $\mathrm{d}_{16}{ }^{15} \mathrm{~N}$ and L-tryptophan . . . 58

4.6. Calculated range of the distance of closest approach . . . . . . . . . . 61

4.7. Calculated enhancements of L-tryptophan- $\mathrm{d}_{8},{ }^{15} \mathrm{~N}_{2},{ }^{13} \mathrm{C}_{11} \ldots \ldots$. . . . 64

4.8. Calculated enhancements of L-tryptophan- ${ }^{15} \mathrm{~N}_{2},{ }^{13} \mathrm{C}_{11} \ldots \ldots$. . . . . . 69 


\section{A.3. List of Figures}

1.1. Energy states of a two-spin system . . . . . . . . . . . . 9 9

1.2. Dependency of the coupling factor on the proton lamor frequency . . 19

2.1. Schematic overview of the $\mathrm{DNP}$ shuttle spectrometer . . . . . . . 22

2.2. The magnetic field profile between the low field plateau and the high field center . . . . . . . . . . . . . . . . . 2 23

2.3. Overview of the EPR cavity (longitudinal cross section) . . . . . 2 25

2.4. Magnetic and electric field profile of the $\mathrm{TM}_{110}$ mode in the EPR cavity 26

2.5. Scheme of the shuttle container design . . . . . . . . 2 28

2.6. Shuttle timing diagram . . . . . . . . . . . . 30

2.7. Overview of the NMR probe . . . . . . . . . . . . . . . 33

3.1. Dependence of the chemical shift of DSS and water on the temperature 37

3.2. Dependence of the output power of $\mathrm{mm}$ amplifier on the attenuation of the E-Scan mw output signal . . . . . . . . . . . . . . . 38

4.1. ${ }^{1} \mathrm{H} \mathrm{DNP}$ enhancement of L-tryptophan . . . . . . . . . . . 43

4.2. Applied global enhancement factor of $\mathrm{H}^{\delta 1}$ of L-tryptophan . . . . . 53

4.3. Dependency of the coupling factor on the distance of closest approach for L-tryptophan . . . . . . . . . . . . . . . 60

4.4. ${ }^{13} \mathrm{C}$ enhancements of L-tryptophan- $\mathrm{d}_{8},{ }^{15} \mathrm{~N}_{2},{ }^{13} \mathrm{C}_{11} \ldots \ldots \ldots \ldots 64$

4.5. ${ }^{13} \mathrm{C}$ enhancements of L-tryptophan $-{ }^{15} \mathrm{~N}_{2},{ }^{13} \mathrm{C}_{11} \ldots \ldots \ldots 6$

4.6. Dependency of the proton coupling factor on the distance of closest approach for Ubiquitin-U- ${ }^{15} \mathrm{~N}, \mathrm{U}-{ }^{13} \mathrm{C} \ldots \ldots . . .70 .70$

4.7. ${ }^{1} \mathrm{H}_{-}{ }^{13} \mathrm{C}-\mathrm{HSQC}$ spectrum of Ubiquitin- $\mathrm{U}_{-}{ }^{15} \mathrm{~N}, \mathrm{U}_{-}{ }^{13} \mathrm{C} \ldots \ldots \ldots . .72$

4.8. Shuttle and $\overline{\mathrm{DNP}}{ }^{1} \mathrm{H}_{-}{ }^{13} \mathrm{C}-\mathrm{HSQC}$ spectrum of Ubiquitin- $\mathrm{U}_{-}{ }^{15} \mathrm{~N}, \mathrm{U}_{-}{ }^{13} \mathrm{C}$. 73 


\section{B. Literature}

[1] A. Krahn, P. Lottmann, T. Marquardsen, A. Tavernier, M.-T. Tuerke, M. Reese, A. Leonov, M. Bennati, P. Hoefer, F. Engelke and C. Griesinger, Phys. Chem. Chem. Phys., 2010, 12, 5830.

[2] P. Lottmann, T. Marquardsen, A. Krahn, A. Tavernier, P. Hoefer, M. Bennati, F. Engelke and C. Griesinger, Appl. Magn. Reson., 2012, 43, 207.

[3] M. H. Levitt, Spin Dynamics: Basics of Nuclear Magnetic Resonance, John Wiley \& Sons, 2007.

[4] J. Keeler, Understanding NMR Spectroscopy, John Wiley \& Sons, 2005.

[5] J. Cavanagh, W. J. Fairbrother, A. G. P. III, M. Rance and N. J. Skelton, Protein NMR Spectroscopy, Academic Press, 2nd edn., 2005.

[6] K. Hausser and D. Stehlik, Adv. Magn. Reson., 1968, 3, 79.

[7] R. G. Griffin and T. F. Prisner, Phys. Chem. Chem. Phys., 2010, 12, 5737.

[8] R. R. Ernst and W. A. Anderson, Rev. Sci. Instrum., 1966, 37, 93.

[9] P. Styles, N. Soffe, C. Scott, D. Cragg, F. Row, D. White and P. White, J. Magn. Reson., 1984, 60, 397.

[10] S. R. Hartmann and E. L. Hahn, Phys. Rev., 1962, 128, 2042.

[11] A. Pines, M. G. Gibby and J. S. Waugh, J. Chem. Phys., 1972, 56, 1776.

[12] G. Morris and R. Freeman, J. Am. Chem. Soc., 1979, 101, 760.

[13] G. Bodenhausen and D. J. Ruben, Chem. Phys. Lett., 1980, 69, 185. 
[14] C. P. Slichter, Phys. Chem. Chem. Phys., 2010, 12, 5741.

[15] A. Overhauser, Phys. Rev., 1953, 92, 476.

[16] T. R. Carver and C. P. Slichter, Phys. Rev., 1953, 92, 212.

[17] I. Solomon, Phys. Rev., 1955, 99, 559.

[18] T. R. Carver and C. P. Slichter, Phys. Rev., 1956, 102, 975.

[19] T. Prisner and W. Kockenberger, Appl. Magn. Reson., 2008, 34, 213.

[20] A. V. Atsarkin and W. Koeckenberger, Appl. Magn. Reson., 2012, 43, 1.

[21] L. R. Becerra, G. J. Gerfen, R. J. Temkin, D. J. Singel and R. G. Griffin, Phys. Rev. Lett., 1993, 71, 3561.

[22] L. Becerra, G. Gerfen, B. Bellew, J. Bryant, S. Hall, D. Inati, R. Weber, S. Un, T. Prisner, A. McDermott, K. Fishbein, K. Kreischer, R. Temkin, D. Singel and R. Griffin, J. Magn. Reson., 1995, 117, 28.

[23] K.-N. Hu, H.-H. Yu, T. M. Swager and R. G. Griffin, J. Am. Chem. Soc., 2004, 126, 10844.

[24] C. Song, K.-N. Hu, C.-G. Joo, T. M. Swager and R. G. Griffin, J. Am. Chem. Soc., 2006, 128, 11385.

[25] T. Maly and J. R. Sirigiri, Appl. Magn. Reson., 2012, 43, 181.

[26] C. Sauvee, M. Rosay, G. Casano, F. Aussenac, R. T. Weber, O. Ouari and P. Tordo, Angew. Chem., 2013, 125, 11058.

[27] K.-N. Hu, G. T. Debelouchina, A. A. Smith and R. G. Griffin, J. Chem. Phys., 2011, 134, 125105.

[28] Y. Hovav, O. Levinkron, A. Feintuch and S. Vega, Appl. Magn. Reson., 2012, $43,21$.

[29] S. Jannin, A. Comment and J. J. van der Klink, Appl. Magn. Reson., 2012, 43, 59. 
[30] G. T. Debelouchina, M. J. Bayro, P. C. A. van der Wel, M. A. Caporini, A. B. Barnes, M. Rosay, W. E. Maas and R. G. Griffin, Phys., 2010, 12, 5911.

[31] A. S. L. Thankamony, O. Lafon, X. Lu, F. Aussenac, M. Rosay, J. TrÃ@bosc, H. Vezin and J.-P. Amoureux, Appl. Magn. Reson., 2012, 43, 237.

[32] J. Ardenkjaer-Larsen, B. Fridlund, A. Gram, G. Hansson, L. Hansson, M. Lerche, R. Servin, M. Thaning and K. Golman, Proc. Natl. Acad. Sci. U. S. A., 2003, 100, 10158.

[33] S. Jannin, A. Bornet, R. Melzi and G. Bodenhausen, Chem. Phys. Lett., 2012, $\mathbf{5 4 9}, 99$.

[34] A. Bornet, R. Melzi, A. J. P. Linde, P. Hautle, B. van den Brandt, S. Jannin and G. Bodenhausen, J. Phys. Chem. Lett., 2013, 4, 111.

[35] K. Golman, R. Zandt and M. Thanning, Proc. Natl. Acad. Sci. U. S. A., 2006, 103, 11270.

[36] S. E. Day, M. I. Kettunen, F. A. Gallagher, D.-E. Hu, M. Lerche, J. Wolber, K. Golman, J. H. Ardenkjaer-Larsen and K. M. Brindle, Nat. Med. (N. Y., NY, U. S.), 2007, 13, 1382.

[37] S. Bowen and C. Hilty, Phys. Chem. Chem. Phys., 2010, 12, 5766.

[38] M. Ragavan, H.-Y. Chen, G. Sekar and C. Hilty, Anal. Chem., 2011, 83, 6054.

[39] Y. Lee, H. Zeng, A. Mazur, M. Wegstroth, T. Carlomagno, M. Reese, D. Lee, S. Becker, C. Griesinger and C. Hilty, Angew. Chem., Int. Ed., 2012, 51, 5179.

[40] H. Zeng, Y. Lee and C. Hilty, Anal. Chem., 2010, 82, 8897.

[41] H.-Y. Chen, M. Ragavan and C. Hilty, Angew. Chem., Int. Ed., 2013, 52, 9192.

[42] V. Denysenkov, M. Prandolini, A. Krahn, M. Gafurov, B. Endeward and T. Prisner, Appl. Magn. Reson.denysenkov08, 2008, 34, 289.

[43] M. Prandolini, V. Denysenkov, M. Gafurov, S. Lyubenova, B. Endeward, M. Bennati and T. Prisner, Appl. Magn. Reson., 2008, 34, 399. 
[44] V. Denysenkov, M. J. Prandolini, M. Gafurov, D. Sezer, B. Endeward and T. F. Prisner, Phys. Chem. Chem. Phys., 2010, 12, 5786.

[45] P. Neugebauer, J. G. Krummenacker, V. P. Denysenkov, G. Parigi, C. Luchinat and T. F. Prisner, Phys. Chem. Chem. Phys., 2013, 15, 6049.

[46] J. G. Krummenacker, V. Denysenkov and T. F. Prisner, Appl. Magn. Reson., 2012, 43, 139 .

[47] M. Reese, D. Lennartz, T. Marquardsen, P. Hoefer, A. Tavernier, P. Carl, T. Schippmann, M. Bennati, T. Carlomagno, F. Engelke and C. Griesinger, Appl. Magn. Reson., 2008, 34, 301.

[48] M. Reese, M. Tuerke, I. Tkach, G. Parigi, C. Luchinat, T. Marquardsen, A. Tavernier, P. Hoefer, F. Engelke and C. Griesinger, J. Am. Chem. Soc., 2009, 131, 15086.

[49] A. Abragam, Nuclear Magnetism, Oxford Univ. Press, London and New York, 1961.

[50] M. Bennati, C. Luchinat, G. Parigi and M.-T. Tuerke, Phys. Chem. Chem. Phys., 2010, 12, 5902.

[51] F. Bloch, Phys. Rev., 1946, 70, 460.

[52] M.-T. Tuerke, I. Tkach, M. Reese, P. Hoefer and M. Bennati, Phys. Chem. Chem. Phys., 2010, 12, 5893.

[53] G. E. Pake and J. T. R. Tuttle, Phys. Rev. Lett., 1959, 3, 423.

[54] J. D. Currin, Phys. Rev., 1962, 126, 1995.

[55] J. H. Freed, J. Chem. Phys., 1965, 43, 2312.

[56] J. H. Freed, J. Phys. Chem., 1967, 71, 38.

[57] M.-T. Tuerke and M. Bennati, Phys. Chem. Chem. Phys., 2011, 13, 3630.

[58] M.-T. Tuerke, G. Parigi, C. Luchinat and M. Bennati, Phys. Chem. Chem. Phys., 2012, 14, 502. 
[59] N. Bloembergen, J. Chem. Phys., 1957, 27, 572.

[60] C. F. Polnaszek and R. G. Bryant, J. Chem. Phys., 1984, 81, 4038.

[61] H. F. Bennett, R. D. B. III, S. H. Koenig and H. M. Swartz, Magn. Reson. Med., 1987, 4, 93 .

[62] L.-P. Hwang and J. H. Freed, J. Chem. Phys., 1975, 63, 4017.

[63] J. H. Freed, J. Chem. Phys., 1978, 68, 4034.

[64] N. Bloembergen and L. O. Morgan, J. Chem. Phys., 1961, 34, 842.

[65] Z. Luz and S. Meiboom, J. Chem. Phys., 1964, 40, 2686.

[66] B. Bates, M. Johnson and B. Currie, J. Labelled Compd. Radiopharm., 1983, 20, 33 .

[67] K. Yamada, Y. Kinoshita, T. Yamasaki, H. Sadasue, F. Mito, M. Nagai, S. Matsumoto, M. Aso, H. Suemune, K. Sakai and H. Utsumi, Arch. Pharm. Chem. Life Sci., 2008, 341, 548.

[68] E. C. Johnson, G. A. Lazar, J. R. Desjarlais and T. M. Handel, Structure, 1999, 7, 976 .

[69] R. E. Hoffman, Magn. Reson. Chem., 2006, 44, 606.

[70] M. Liu, X. Mao, C. Ye, H. Huang, J. K. Nicholson and J. C. Lindon, Journal of Magnetic Resonance, 1998, 132, 125.

[71] T.-L. Hwang and A. J. Shaka, J. Magn. Reson. Series A, 1995, 112, 275.

[72] H. Henry-Riyad and T. T. Tidwell, Journal of Physical Organic Chemistry, 2003, 16, 559 .

[73] C. S. J. Jr., Prog. Nucl. Magn. Reson. Spectrosc., 1999, 34, 203.

[74] V. Sklenar, M. Piotto, R. Leppik and V. Saudek, J. Magn. Reson. Series A, 1993, 102, 241. 
[75] F. Delaglio, S. Grzesiek, G. W. Vuister, G. Zhu, J. Pfeifer and A. Bax, J. Biomol. NMR, 1995, 6, 277.

[76] R. L. J. Keller, The Computer Aided Resonance Assigment Tutorial, Cantina Verlag, 2004.

[77] P. Hoefer, P. Carl, G. Guthausen, T. Prisner, M. Reese, T. Carlomagno, C. Griesinger and M. Bennati, Appl. Magn. Reson., 2008, 34, 393.

[78] V. Vitzthum, F. Borcard, S. Jannin, M. Morin, P. Mieville, M. A. Caporini, A. Sienkiewicz, S. Gerber-Lemaire and G. Bodenhausen, ChemPhysChem, 2011, 12, 2929 .

[79] D. Rovnyak, J. Hoch, A. Stern and G. Wagner, J. Biomol. NMR, 2004, 30, 1.

[80] C. Luchinat and G. Parigi, Appl. Magn. Reson., 2008, 34, 379.

[81] D. Sezer, M. J. Prandolini and T. F. Prisner, Phys. Chem. Chem. Phys., 2009, 11, 6626 .

[82] L. G. Longsworth, J. Am. Chem. Soc., 1953, 75, 5705.

[83] C. H. Cho, J. Urquidi, S. Singh and G. W. Robinson, J. Phys. Chem. B, 1999, 103, 1991.

[84] I. Peric, D. Merunka, B. L. Bales and M. Peric, J. Phys. Chem. Lett., 2013, 4, 508.

[85] L. X.-Q. Chen, R. A. Engh and G. R. Fleming, J. Phys. Chem., 1988, 92, 4811.

[86] D. Sezer, Phys. Chem. Chem. Phys., 2013, 15, 526.

[87] D. F. S. Natusch, R. E. Richards and D. Taylor, Mol. Phys., 1966, 11, 421.

[88] J. R. Stewart, J. Chem. Phys., 1971, 54, 492.

[89] G. Cornilescu, J. L. Marquardt, M. Ottiger and A. Bax, J. Am. Chem. Soc., 1998, 120, 6836 . 
[90] E. L. Ulrich, H. Akutsu, J. F. Doreleijers, Y. Harano, Y. E. Ioannidis, J. Lin, M. Livny, S. Mading, D. Maziuk, Z. Miller, E. Nakatani, C. F. Schulte, D. E. Tolmie, R. K. Wenger, H. Yao and J. L. Markley, Nucleic Acids Res., 2008, 36, D402.

[91] K. H. Mayo, E. Ilyina and H. Park, Protein Sci., 1996, 5, 1301.

[92] N. Tjandra, S. E. Feller, R. W. Pastor and A. Bax, J. Am. Chem. Soc., 1995, 117, 12562 . 



\section{Pulse programs and scripts}

\section{C.1. Pulse programs}

In the following section, the pulse sequences are given, which were used for the NMR and DNP experiments on the DNP shuttle spectrometer and which significantly deviate from the pulse programs provide by Bruker.

Pulse program for ${ }^{1} H \overline{D N P}$ experiments with watergate (w5) sequence for water suppression:

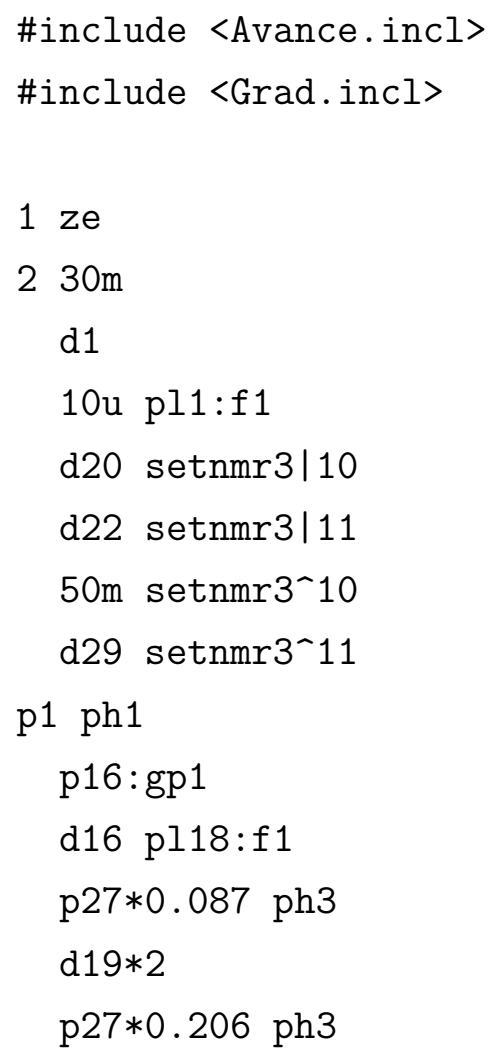


$\mathrm{d} 19 * 2$

p27*0.413 ph3

$\mathrm{d} 19 * 2$

p27*0.778 ph3

d $19 * 2$

p27*1.491 ph3

$\mathrm{d} 19 * 2$

p27*1.491 ph4

$\mathrm{d} 19 * 2$

p27*0.778 ph4

$\mathrm{d} 19 * 2$

p27*0.413 ph 4

$\mathrm{d} 19 * 2$

p27*0.206 ph4

$\mathrm{d} 19 * 2$

p27*0.087 ph4

$50 \mathrm{u}$

$\mathrm{p} 16: \mathrm{gp} 1$

d16

$4 \mathrm{u}$

$\mathrm{p} 16: \mathrm{gp} 2$

d16

p27*0.087 ph5

$\mathrm{d} 19 * 2$

p27*0.206 ph5

$\mathrm{d} 19 * 2$

p27*0.413 ph5

$\mathrm{d} 19 * 2$

p27*0.778 ph5

d $19 * 2$

p27*1.491 ph5

d $19 * 2$

p27*1.491 ph6 


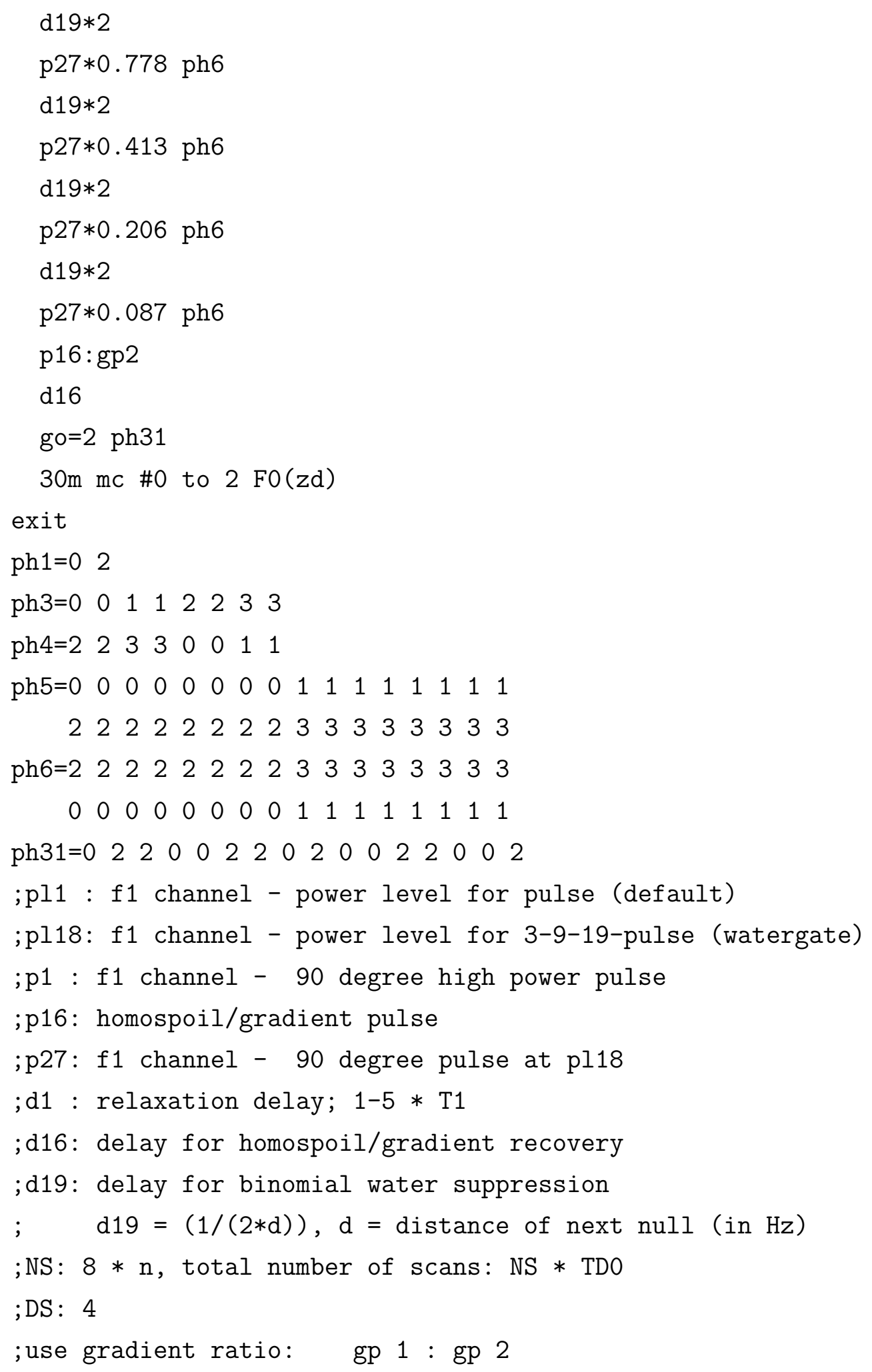


; $34: 22$

; for z-only gradients:

;gpz1: $34 \%$

;gpz2: $22 \%$

; use gradient files:

;gpnam1: SINE.100

;gpnam2: SINE.100

Pulse program for ${ }^{1} H D N P$ experiments with gradients and excitation sculpting for water suppression:

\#include <Avance.incl>

\#include <Grad.incl>

\#include <Delay.incl>

"p2=p1*2"

"d12=20u"

"d29=d28-50m"

"TAU $=\mathrm{de}+\mathrm{p} 1 * 2 / 3 \cdot 1416+50 \mathrm{u} "$

"acqt $0=0$ "

baseopt_echo

$1 \mathrm{ze}$

50u UNBLKGRAD

$230 \mathrm{~m}$

d2

d12 pl1:f1

d20 setnmr3/10

d22 setnmr3|11

$50 \mathrm{~m}$ setnmr3 10

d29 setnmr3^11

p1 ph1

$50 \mathrm{u}$

$\mathrm{p} 16: \mathrm{gp} 1$ 


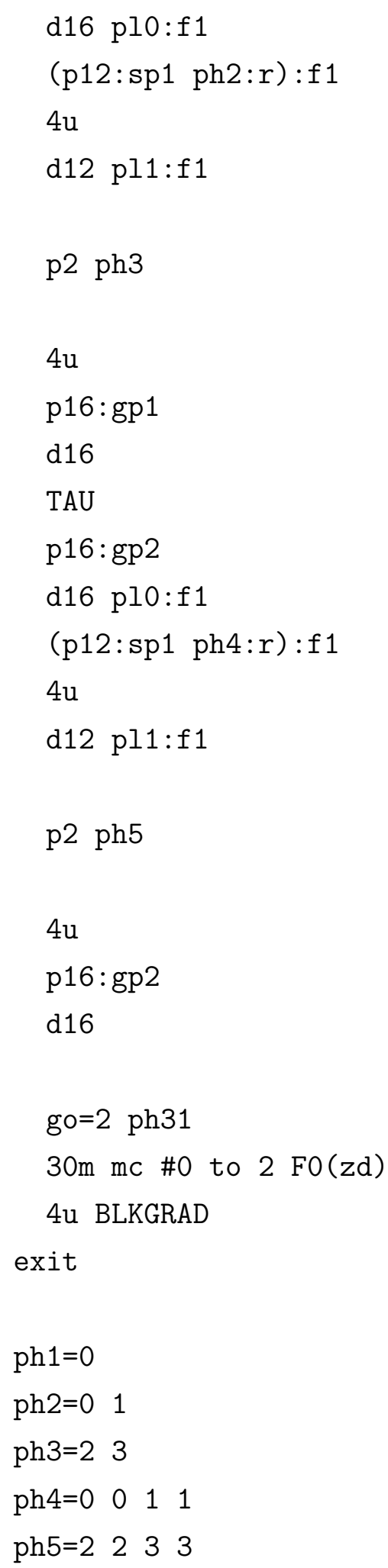


ph31=0 2220

;p10 : OW

;pl1 : f1 channel - power level for pulse (default)

;sp1 : f1 channel - shaped pulse 180 degree

;p1 : f1 channel - 90 degree high power pulse

;p2 : f1 channel - 180 degree high power pulse

;p12: f1 channel - 180 degree shaped pulse (Squa100.1000) [2 msec]

;p16: homospoil/gradient pulse

; d1 : relaxation delay; $1-5 * \mathrm{~T} 1$

;d12: delay for power switching

[20 usec]

;d16: delay for homospoil/gradient recovery

;NS: $8 * \mathrm{n}$, total number of scans: NS * TDO

;DS : 4

; use gradient ratio: $\quad$ gp 1 : gp 2

; $\quad 31: 11$

;for z-only gradients:

;gpz1: $31 \%$

;gpz2: $11 \%$

; use gradient files:

;gpnam1: SMSQ10.100

;gpnam2: SMSQ10.100

Pulse program for ${ }^{13} \mathrm{C} D N P$ experiments with additional lock delay and proton decoupling:

\#include <Avance.incl>

\#include <Grad.incl>

\#include <Delay.incl>

"d11=30m"

"d29=d28-50m"

"acqt $0=-\mathrm{p} 1 * 2 / 3.1416$ " 
$1 \mathrm{ze}$

d11 pl12:f2

$230 \mathrm{~m}$

d2

d1 LOCKH_OFF

500m LOCKH_ON

d20 setnmr3|10

d22 setnmr3|11

$50 \mathrm{~m}$ setnmr 3 - 10

d29 setnmr3^11

p1 ph1

go=2 ph31

$30 \mathrm{~m} \mathrm{mc} \# 0$ to $2 \mathrm{FO}(\mathrm{zd})$

exit

ph1=0 $\begin{array}{llllllll}2 & 2 & 0 & 1 & 3 & 3 & 1\end{array}$

ph31=0 $2 \begin{array}{llllll}2 & 0 & 1 & 3 & 3 & 1\end{array}$

;pl0 : OW

;pl1 : f1 channel - power level for pulse (default)

;pl12: f2 channel - power level for CPD/BB decoupling

;p1 : f1 channel - high power pulse

;d1 : relaxation delay; 1-5 * T1

;d11: delay for disk I/O

$[30 \mathrm{msec}]$

;NS: $8 * \mathrm{n}$, total number of scans: NS $*$ TDO

; cpd2: decoupling according to sequence defined by cpdprg2

;pcpd2: f2 channel - 90 degree pulse for decoupling sequence

Pulse program for ${ }^{13} \mathrm{C} D N P$ experiments with additional lock delay and proton decoupling - version 2:

\#include <Avance.incl>

\#include <Grad.incl>

\#include <Delay.incl> 


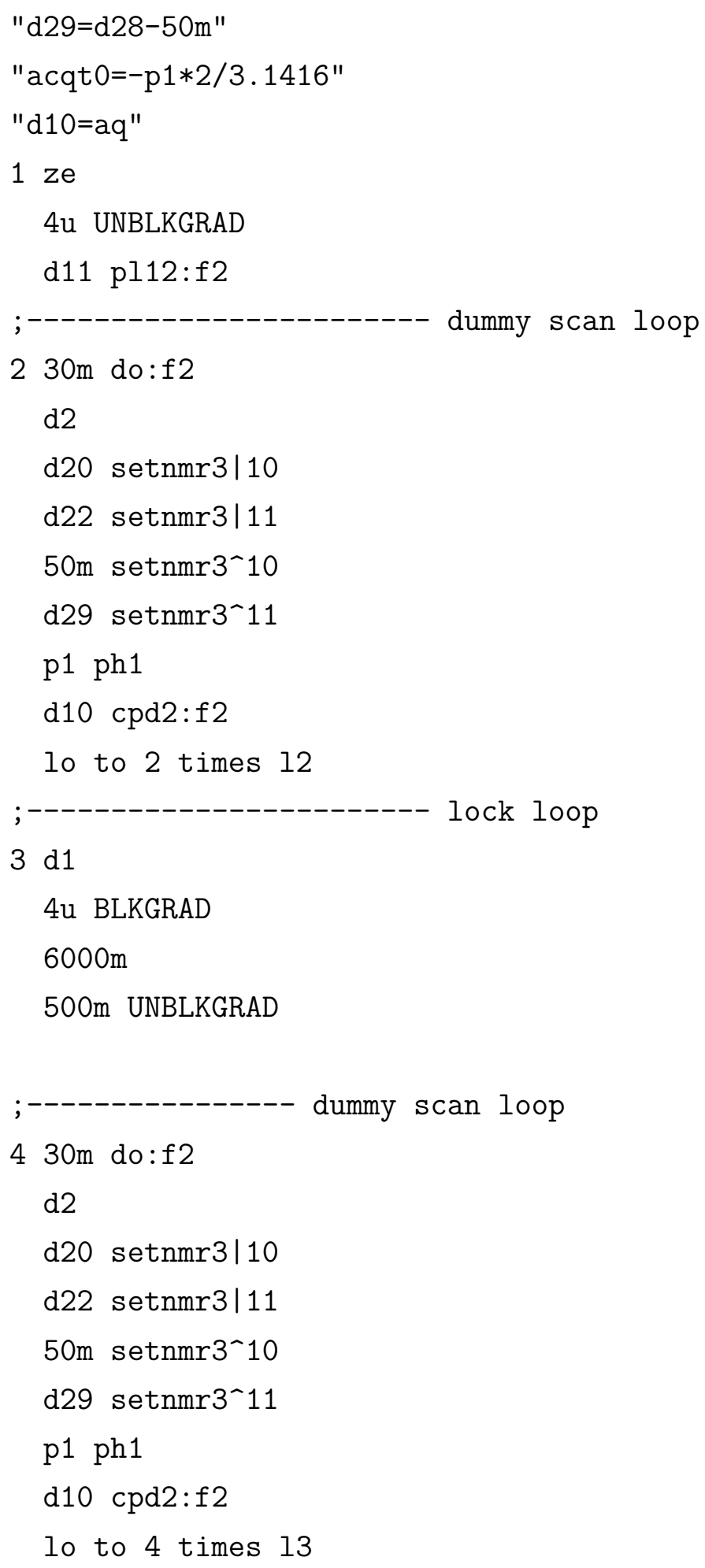


$530 \mathrm{~m}$ do: $\mathrm{f} 2$

$\mathrm{d} 2$

d20 setnmr3|10

d22 setnmr3|11

$50 \mathrm{~m}$ setnmr $3{ }^{\wedge} 10$

d29 setnmr3^11

p1 ph1

go $=5$ ph31 cpd2:f2

$500 \mathrm{~m}$

lo to 3 times 11

30m do:f2 mc \#0 to $2 \mathrm{FO}(\mathrm{zd})$

$4 \mathrm{u}$ BLKGRAD

exit

ph1=0 $2 \begin{array}{lllllll}2 & 0 & 1 & 3 & 3 & 1\end{array}$

ph31=0 $22 \begin{array}{llllll} & 0 & 1 & 3 & 3 & 1\end{array}$

;p10 : OW

;pl1 : f1 channel - power level for pulse (default)

;pl12: f2 channel - power level for CPD/BB decoupling

;p1 : f1 channel - high power pulse

;p16: homospoil/gradient pulse

; 11 : relaxation delay; $1-5 * \mathrm{~T} 1$

;d11: delay for disk I/O

[30 $\mathrm{msec}]$

;d12: delay for power switching

[20 usec]

;d16: delay for homospoil/gradient recovery

;NS: $8 * \mathrm{n}$, total number of scans: NS $*$ TDO

; cpd2: decoupling according to sequence defined by cpdprg2

;pcpd2: f2 channel - 90 degree pulse for decoupling sequence 
Pulse program for $H S Q C$ with Boltzmann polarization:






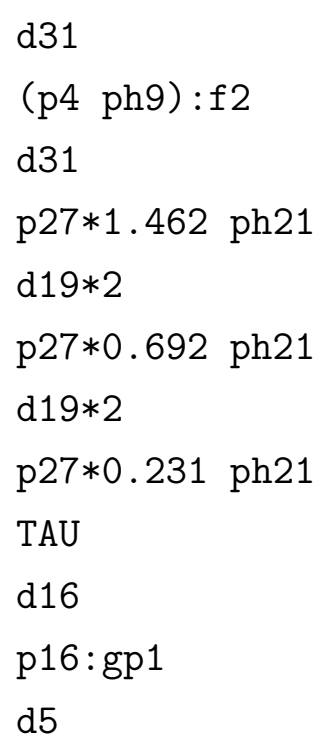




$$
\begin{aligned}
& (p 4 \text { ph5):f2 } \\
& \text { d31 } \\
& \text { p27*1.462 ph23 } \\
& d 19 * 2 \\
& \text { p27*0.692 ph23 } \\
& d 19 * 2 \\
& \text { p27*0.231 ph23 }
\end{aligned}
$$

TAU

d16 pl12:f2

$\mathrm{p} 16: \mathrm{gp} 2$

d5

\section{go=2 ph31 cpd2:f2}

d1 do:f2 mc \#0 to 2 F1PH(calph(ph6, +90) \& calph(ph9, +90), $\operatorname{caldel}(d 0,+i n 0))$

$4 \mathrm{u}$ BLKGRAD

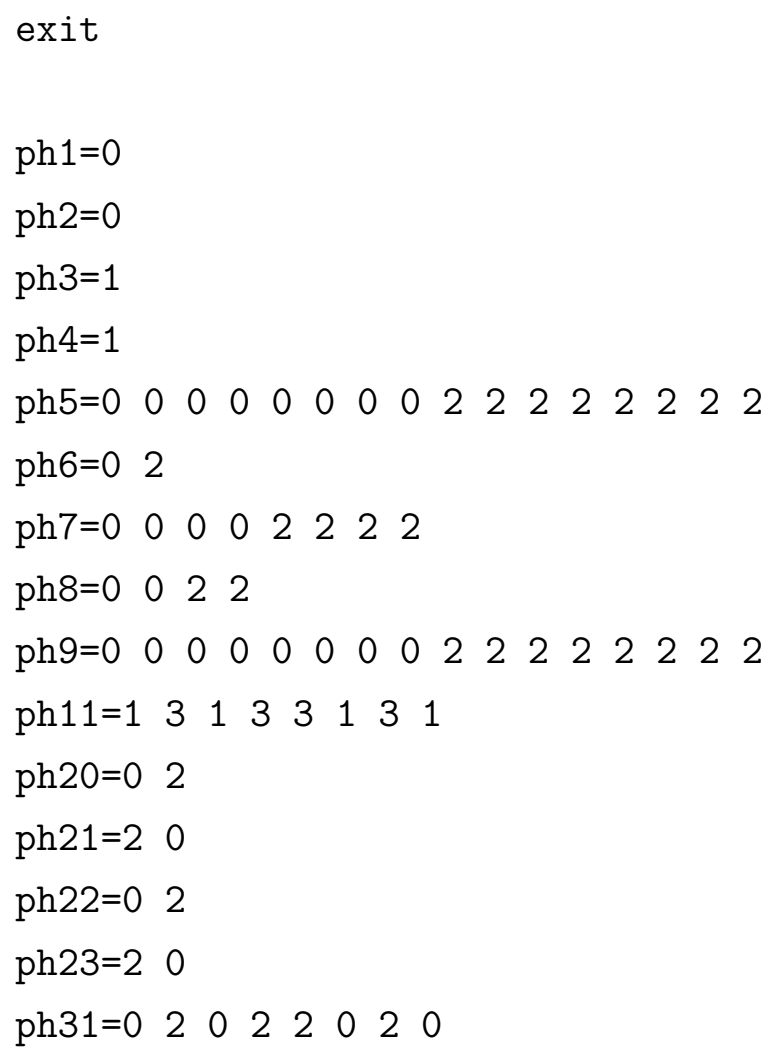




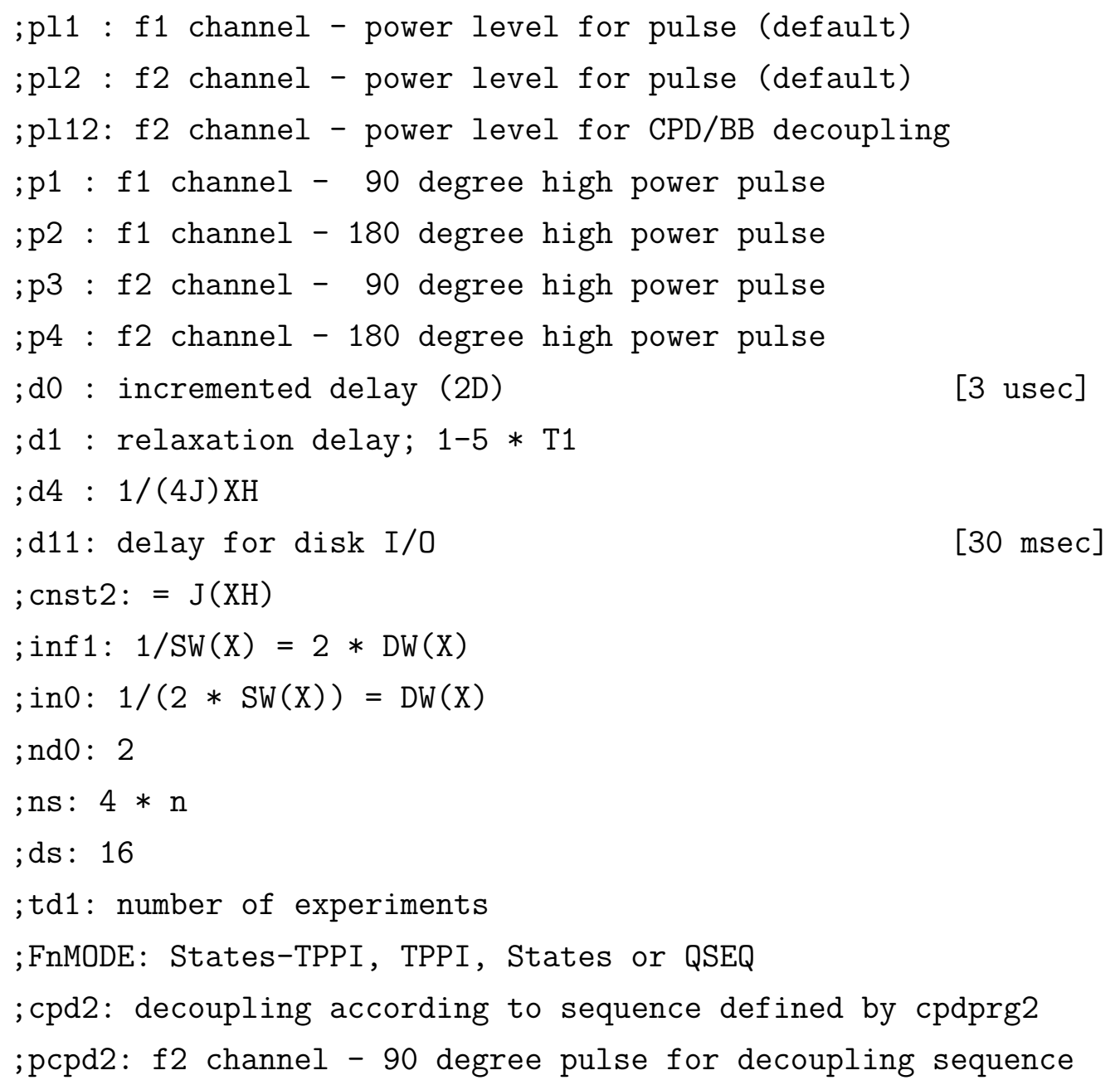

Pulse program for $H S Q C$ with Boltzmann polarization and shuttling:

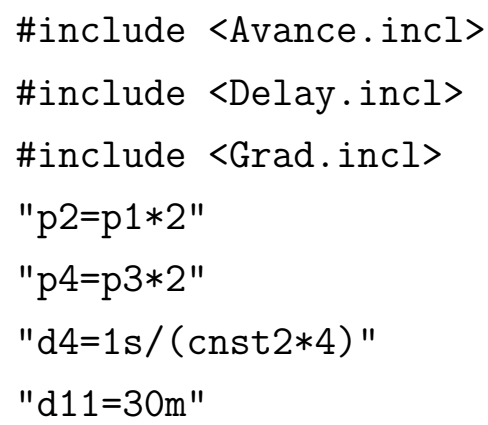




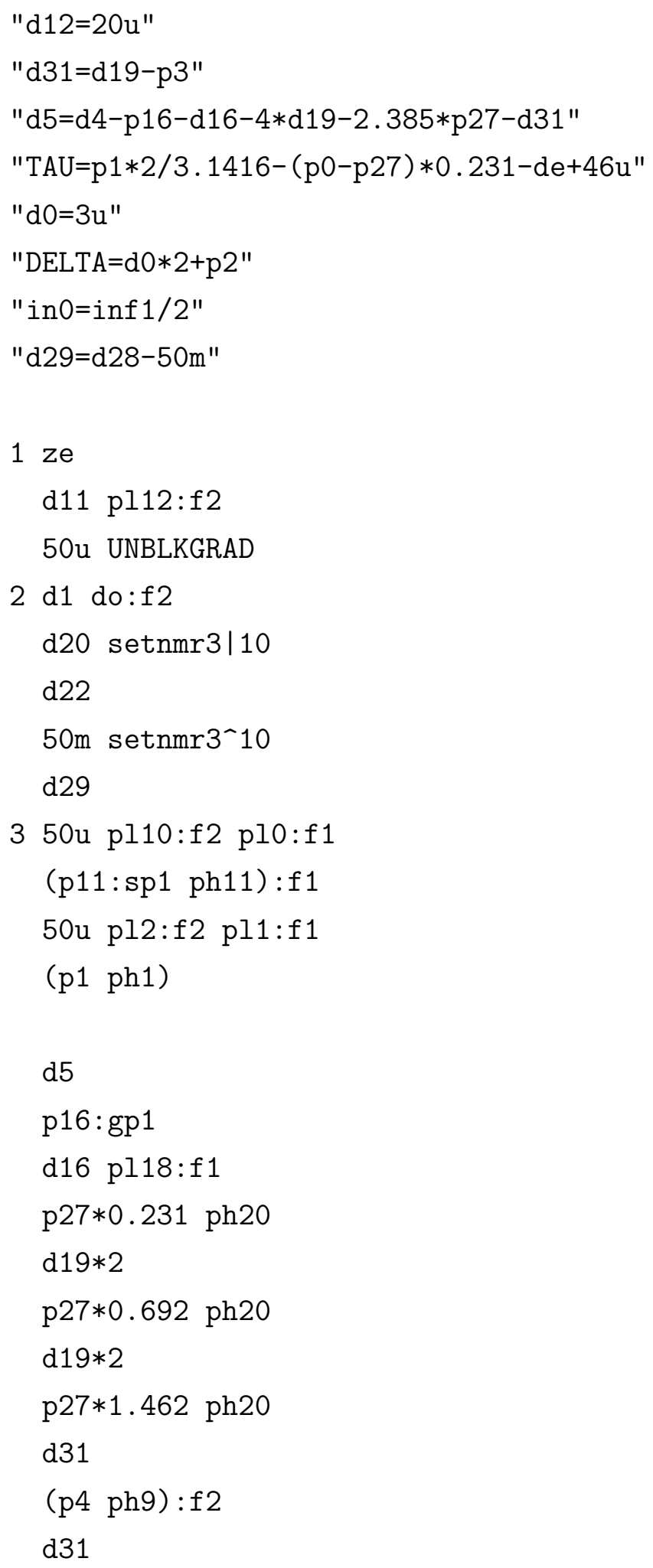




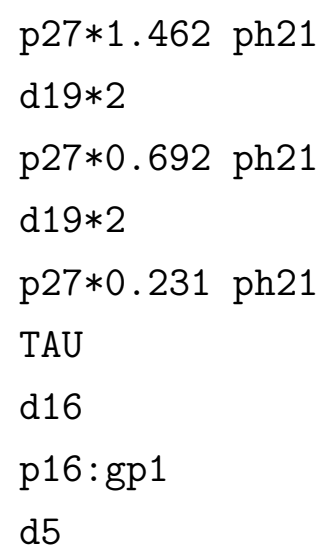




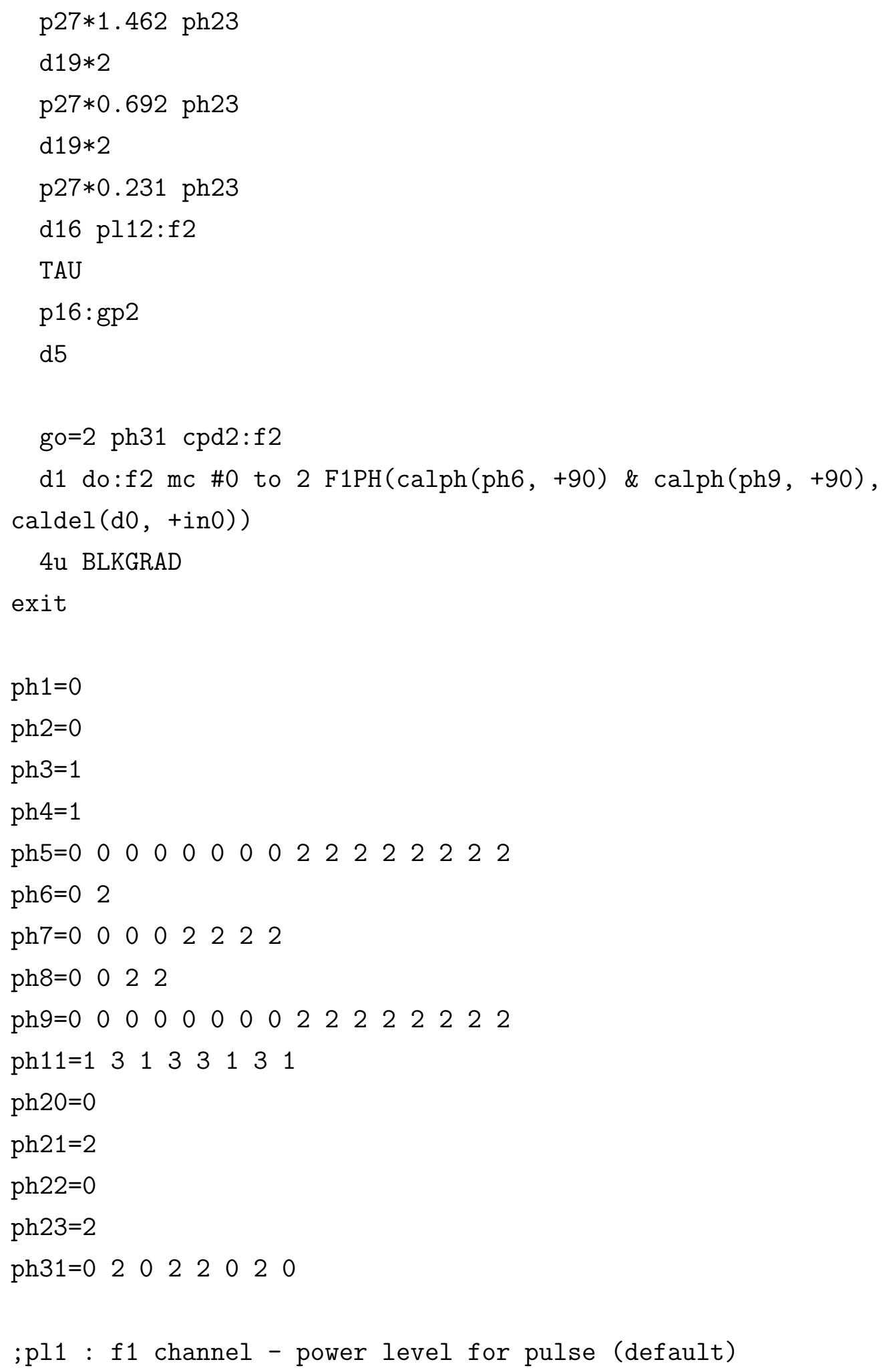


;p12 : f2 channel - power level for pulse (default)

;pl12: f2 channel - power level for CPD/BB decoupling

;p1 : f1 channel - 90 degree high power pulse

;p2 : f1 channel - 180 degree high power pulse

;p3 : f2 channel - 90 degree high power pulse

;p4 : f2 channel - 180 degree high power pulse

; d0 : incremented delay (2D)

[3 usec]

; $\mathrm{d} 1$ : relaxation delay; $1-5 * \mathrm{~T} 1$

; d4 : 1/(4J) XH

;d11: delay for disk I/O

[30 $\mathrm{msec}]$

; cnst2: $=\mathrm{J}(\mathrm{XH})$

$; \inf 1: 1 / \mathrm{SW}(\mathrm{X})=2 * \mathrm{DW}(\mathrm{X})$

;in0: $1 /(2 * \operatorname{SW}(X))=\operatorname{DW}(X)$

;nd0: 2

;ns: $4 * \mathrm{n}$

;ds : 16

;td1: number of experiments

;FnMODE: States-TPPI, TPPI, States or QSEQ

; cpd2: decoupling according to sequence defined by cpdprg2

;pcpd2: f2 channel - 90 degree pulse for decoupling sequence

\section{Pulse program for $H S Q C$ with DNP:}

\#include <Avance.incl>

\#include <Delay.incl>

\#include <Grad.incl>

"p2=p1*2"

"p4=p3*2"

"d $4=1 \mathrm{~s} /($ cnst $2 * 4)$ "

"d11=30m"

"d12=20u" 


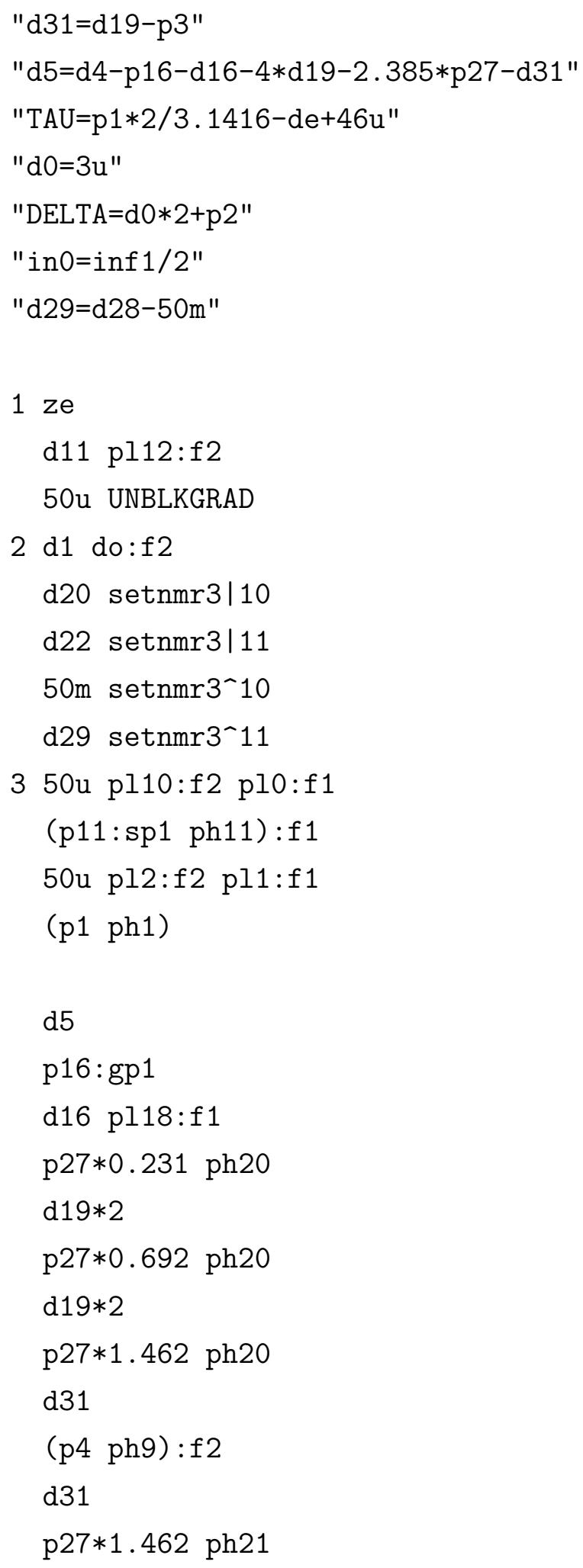




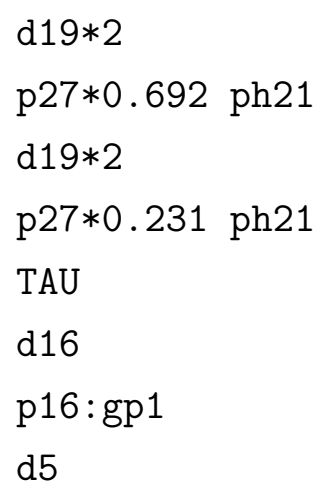




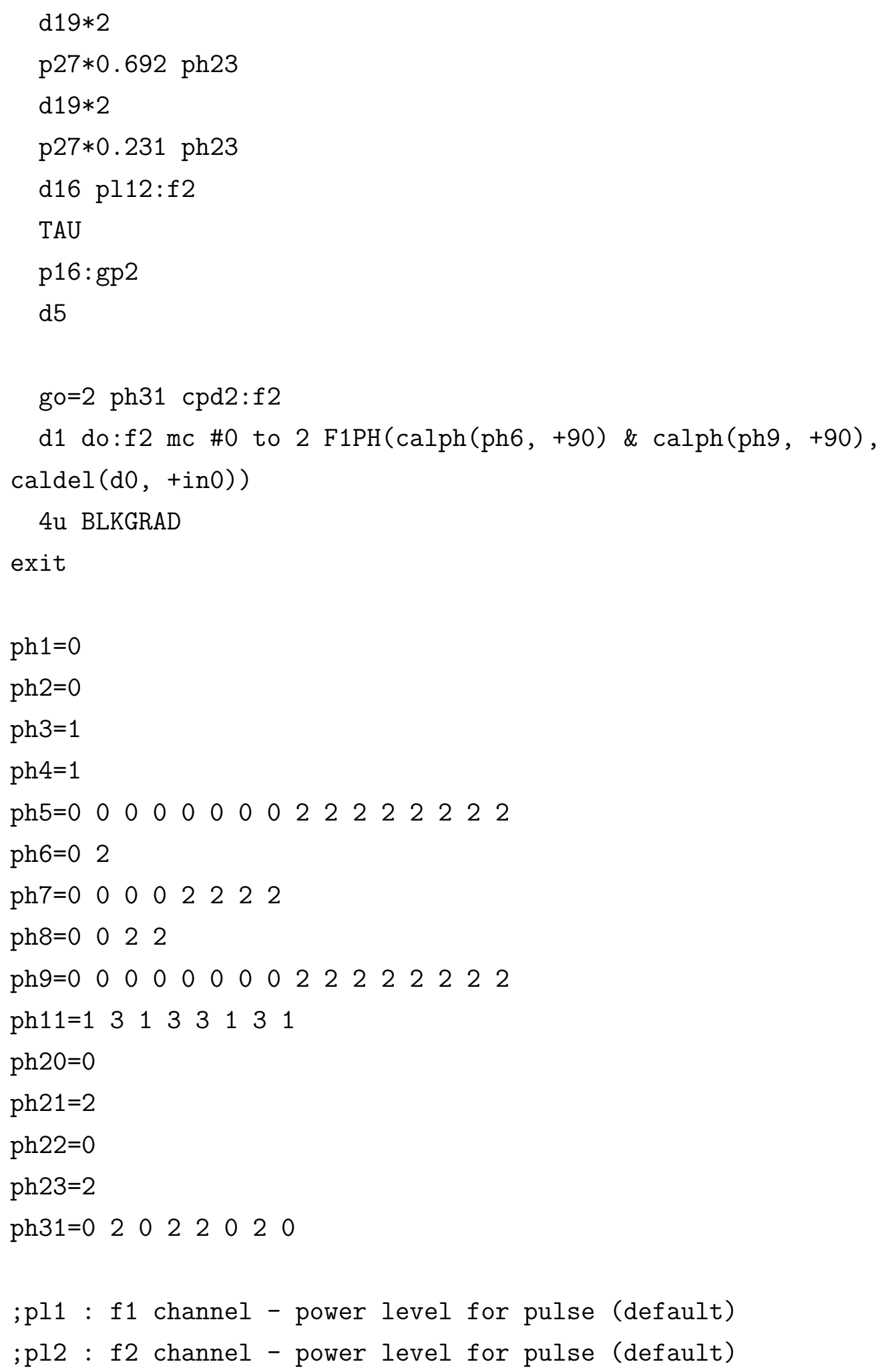


;pl12: f2 channel - power level for CPD/BB decoupling

;p1 : f1 channel - 90 degree high power pulse

;p2 : f1 channel - 180 degree high power pulse

;p3 : f2 channel - 90 degree high power pulse

;p4 : f2 channel - 180 degree high power pulse

;d0 : incremented delay (2D)

[3 usec]

; 11 : relaxation delay; $1-5 * \mathrm{~T} 1$

; d4 : 1/(4J)XH

;d11: delay for disk $1 / 0$

[30 $\mathrm{msec}]$

; cnst2: $=\mathrm{J}(\mathrm{XH})$

;inf 1: $1 / \mathrm{SW}(\mathrm{X})=2 * \mathrm{DW}(\mathrm{X})$

;ino: $1 /(2 * \operatorname{SW}(\mathrm{X}))=\mathrm{DW}(\mathrm{X})$

;nd0: 2

;ns: $4 * \mathrm{n}$

;ds: 16

;td1: number of experiments

;FnMODE: States-TPPI, TPPI, States or QSEQ

; cpd2: decoupling according to sequence defined by cpdprg2 ;pcpd2: f2 channel - 90 degree pulse for decoupling sequence 


\section{C.2. Scilab script}

The following script was used to calculate all distances and coupling factors based on the translational diffusion model and the combined model.

//calculting xi with rotational and translational contribution funcprot(0)//removes info message about change variables clear

$/ /$ constants

$/ / \mathrm{mu} 0$ - permeability constant $\left[\mathrm{N}^{*} \mathrm{~A}^{-}-2\right]$

$\mathrm{mu} 0=4 * \% \mathrm{pi} * 10^{-}-7$

//avo - Avogadro constant [mol^-1]

avo $=6.02214129 * 10^{\sim} 23$

//gam_H - gyromagnetic ratio of proton $\left[\mathrm{s}^{\wedge}-1 \mathrm{~T}^{-}-1\right]$

gam_H=267.5222005*10^ 6

//gam_C - gyromagnetic ratio of carbon $\left[\mathrm{s}^{\wedge}-1 \mathrm{~T}^{-}-1\right]$

gam_C $=67.28286 * 10^{\sim} 6$

//ge - Lande factor of the electron

ge $=2.0023193043617$

//mub - Bohr magneton [J/T]

$\mathrm{mub}=9.27400968 * 10^{\wedge}-24$

//spin - electron

$s=1 / 2$

$/ / \mathrm{planck}$ constant $[\mathrm{J} * \mathrm{~s}]$

$\mathrm{h}=6.62606957 * 10^{\wedge}-34$

$/ /$ reduced planck constant

hbar $=\mathrm{h} /(2 * \% \mathrm{pi})$

//kb - Boltzmann constant

$\mathrm{kb}=1.380648813 * 10^{\wedge}-23$

$/ /$ 
$/ / d$ - Distance of closest approach [Angstrom]

$d=3.5$

$\mathrm{d} 11=3.52$

$\mathrm{d} 12=3.06$

$\mathrm{d} 21=4.16$

$\mathrm{d} 22=3.89$

$\mathrm{d} 31=4.96$

$\mathrm{d} 32=4.79$

$\mathrm{d} 41=4.09$

$\mathrm{d} 42=3.73$

$\mathrm{d} 51=4.64$

$\mathrm{d} 52=4.4$

$\mathrm{d} 61=5.35$

d62 $=5.19$

//Frequency

$\mathrm{nu}=14.5$

//Temperature [K]

$t=273.15+42$

//Carbon proton distance

d_HC=1.1

$/ / \mathrm{n}$ - nuclei in innersphere

$\mathrm{n}=0$

$/ / r$ - distance between radical and nuc $[\mathrm{m}]$

$/ / \mathrm{r}=\mathrm{d}$

//with (1) or without (0) tau_nuc as tau_M

$/ /$

//Sample properties

$/ /++$ solvent viscosity++

//eta - water viscosity $[\mathrm{Pa} * \mathrm{~s}] / /$ cho99

function eta_H_lit=eta_H_lit $(t)$; eta_H_lit $=802.25336 *((t-225.334)$

$+3.4741 * 10^{\wedge}-3 *(t-225.334)^{\wedge} 2-1.7413 * 10^{\wedge}-5 *(t-225.334)^{\wedge} 3+2.7719$ 
$\left.* 10^{\wedge}-8 *(t-225.334)^{\wedge} 4\right)^{\wedge}-1.53026 / 1000$; endfunction

//eta_D_lit - deuterated water viscosity [Pa*s]//cho99

function eta_D_lit=eta_D_lit(t); eta_D_lit $=885.60402 *((t-231.832)$

$+2.7990 * 10^{\wedge}-3 *(t-231.832)^{\wedge} 2-1.6342 * 10^{\wedge}-5 *(t-231.832){ }^{\wedge} 3$

$\left.+2.9067 * 10^{\wedge}-8 *(t-231.832)^{\wedge} 4\right)^{\wedge}-1.55255 / 1000$; endfunction




r_hydro_nuc=kb*T_D_nuc_lit/(D_nuc_lit $* 6 * \%$ pi $*$ eta_D_nuc_lit)

//Come_Hlex life time assume tau_c nuc

//tau_c_nuc_lit

$/ /$ tau_rotnuc

tau_c_nuc_lit $=2.18 * 10^{-}-11 / / 25 \hat{\mathrm{A}}^{\circ} \mathrm{C} / /$ chen 88

T_tau_c_nuc_lit $=298.15$

eta_tau_c_nuc_lit=eta_H_lit(T_tau_c_nuc_lit)

r_rot_nuc $=\left(\left(\right.\right.$ tau_c_nuc_lit $* 3 * k b * T_{-}$tau_c_nuc_lit $) /(4$

$* \%$ pi*eta_tau_c_nuc_lit) $)^{-}(1 / 3)$

$/ /$ magnetic field

function $\mathrm{B}_{-} \mathrm{O}=\mathrm{B}_{-} \mathrm{O}(\mathrm{d}) ; \mathrm{B}_{-} \mathrm{O}=$ nu $* 10^{-} 6 * 2 * \% \mathrm{pi} / \mathrm{gam}_{-} \mathrm{H}$; endfunction

$\operatorname{disp}\left(" B_{-} 0=\right.$ "+string $\left.\left(B_{-} O(d)\right)\right)$

//eta_D - deuterated water viscosity [Pa*s]

//cho92

function eta_ $D=$ eta_ $D(d)$; eta_ $D=885.60402 *((t-231.832)$

$+2.7990 * 10^{\wedge}-3 *(t-231.832)^{\wedge} 2-1.6342 * 10^{\wedge}-5 *(t-231.832) \wedge 3$

$\left.+2.9067 * 10^{\wedge}-8 *(t-231.832)^{\wedge} 4\right)^{\wedge}-1.55255 / 1000$; endfunction

//D - diffusion constant

function D_nuc=D_nuc $(d) ; D_{-}$nuc $=k b * t /\left(6 * \%\right.$ pi $* r_{-}$hydro_nuc $*$ eta_D $\left.(d)\right)$;

endfunction

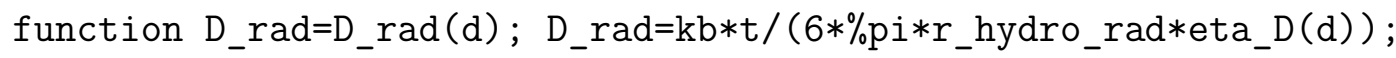

endfunction

//di - relative diffusion coefficient

function D_rel=D_rel(d); D_rel=(D_nuc (d)+D_rad (d)) ; endfunction

$\operatorname{disp}\left(" D_{-}\right.$rad $=$"+string $\left(D_{-}\right.$rad $\left.(d)\right)+" \quad$ " D_nuc $=$ "+string $\left(D_{-}\right.$nuc $\left.(d)\right)$

+" "+"D_rel = "+string (D_rel(d)))

//tau_D - translatioavol correlation time

//for proton 
function tau_D_H=tau_D_H(d); tau_D_H $=\left(d * 10^{-}-10\right)^{\sim} 2 / D_{-} r e l(d)$

; endfunction

//for carbon d plus proton-carbon distance

function tau_D_C=tau_D_C $(d)$; tau_D_C $=\left(\left(d+d_{-} H C\right) * 10^{-}-10\right)$-2/D_rel (d) ;

endfunction

$\operatorname{disp}\left(" t a u_{-} D_{-} H=\right.$ "string $\left(\operatorname{tau}_{-} \mathrm{D}_{-} \mathrm{H}(\mathrm{d})\right)+" \quad$ "+"tau_D_C $=$ "

+string (tau_D_C (d)))

//rotaioavol correlation time

//radical

function tau_c_rad=tau_c_rad (d); tau_c_rad

$=4 * \%$ pi*eta_D $(d) * r_{-} r$ rot_rad^3/(3*kb*t); endfunction

//nucleus

function tau_c_nuc=tau_c_nuc(d); tau_c_nuc

$=4 * \%$ pi $*$ eta_D $(d) * r_{-}$rot_nuc $3 /(3 * \mathrm{~kb} * \mathrm{t})$; endfunction

//tau_rot [s]

function tau_rot=tau_rot (d); tau_rot=(tau_c_rad $(d)^{\wedge}-1$

$\left.+(\text { tau_c_nuc }(d))^{\wedge}-1\right)^{\wedge}-1$; endfunction

$/ /$ tau_rot $[\mathrm{s}]$

//function tau_rot=tau_rot (d); tau_rot $=\left(\operatorname{tau}{ }_{-} c_{-} r a d(d)^{\wedge}-1+t 1 e^{-}-1\right.$

$\left.+b *(\text { tau_c_nuc }(d))^{-}-1\right)^{-}-1$; endfunction

disp $\left(" t a u_{-} r o t="+\right.$ string $($ tau_rot $(d))+" \quad$ "+tau_c_rad $=$ "

+string (tau_c_rad $(d))+" \quad$ "+"tau_c_nuc = "+string $\left(\operatorname{tau}\right.$ s_nuc $\left.\left._{-}(d)\right)\right)$

//factor $k$ for translatioavol contribution

//proton

function $k_{-} t_{-} e H=k_{-} t_{-} e H(d) ; k_{-} t_{-} e H$

$=32 * \%$ pi $/ 405 *(\mathrm{mu0} /(4 * \% \mathrm{pi}))^{\wedge} 2 *$ avo*c_rad $*$ gam_H^ $\mathrm{H}^{\wedge} 2 * \mathrm{ge}^{\wedge} 2 * \mathrm{mub}^{\wedge} 2$

$* s *(s+1) /\left(\left(d * 10^{-}-10\right) * D_{-} r e l(d)\right)$ endfunction 
//carbon

function $k_{-} t_{-} e C=k_{-} t_{-} e C(d) ; k_{-} t_{-} e C$

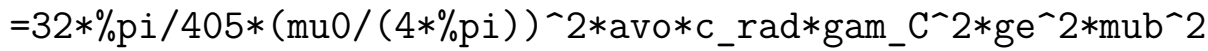

$* s *(\mathrm{~s}+1) /\left(\left(\left(\mathrm{d}+\mathrm{d} \_\mathrm{HC}\right) * 10^{-}-10\right) * \mathrm{D}_{-} \mathrm{rel}(\mathrm{d})\right)$ endfunction

$/ / \mathrm{k}$ for rotational contribution

//electron - proton

function $k_{-} r_{-} e H=k_{-} r_{-} e H(d) ; k_{-} r_{-} e H=n * c_{-} r a d / c_{-} n u c * 2 / 15 *(m u 0 /(4$

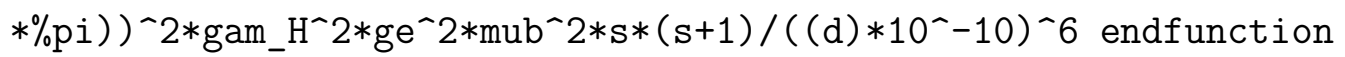

//electron - carbon

function k_r_eC=k_r_eC $(d) ; k_{-} r_{-} e C=n * c_{-} r a d / c_{-} n u c * 2 / 15 *(m u 0 /(4$

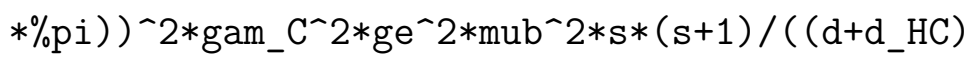

$\left.* 10^{\wedge}-10\right)^{\wedge} 6$ endfunction

$/ /$ proton - carbon

function $\mathrm{k}_{-} \mathrm{r}_{-} \mathrm{HC}=\mathrm{k}_{-} \mathrm{r}_{-} \mathrm{HC}(\mathrm{d}) ; \mathrm{k}_{-} \mathrm{r}_{-} \mathrm{HC}=1 / 10 *(\mathrm{muO} /(4 * \% \mathrm{pi}))^{-} 2$

$*$ gam_C^ $2 *$ gam_H^2 $2 * \operatorname{hbar}^{\wedge} 2 /\left(d_{-} H C * 10^{\wedge}-10\right)^{\wedge} 6$ endfunction

$\operatorname{disp}\left(" k_{-} t_{-} e H=\right.$ "+string $\left(k_{-} t_{-} e H(d)\right)+" \quad "+" k_{-} t_{-} e C="$

+string $\left(k_{-} t_{-} e C(d)\right)+" \quad "+" k_{-} r_{-} e H=~ "+\operatorname{string}\left(k_{-} r_{-} e H(d)\right)+"$ "

$+" k_{-} r_{-} e C=$ +string $\left(k_{-} r_{-} e C(d)\right)+" \quad "+" k_{-} r_{-} H C="$

$\left.+\operatorname{string}\left(k_{-} r_{-} H C(d)\right)\right)$

//omega_electron

function ome_e=ome_e (d); ome_e=ge $*$ mub/hbar $* B_{-} 0(d)$

; endfunction

//omega_proton

function ome_H=ome_H(d); ome_H=gam_H*B_O(d)

; endfunction

//omega_carbon

function ome_C=ome_C $(d)$; ome_C=gam_C*B_O(d)

; endfunction 


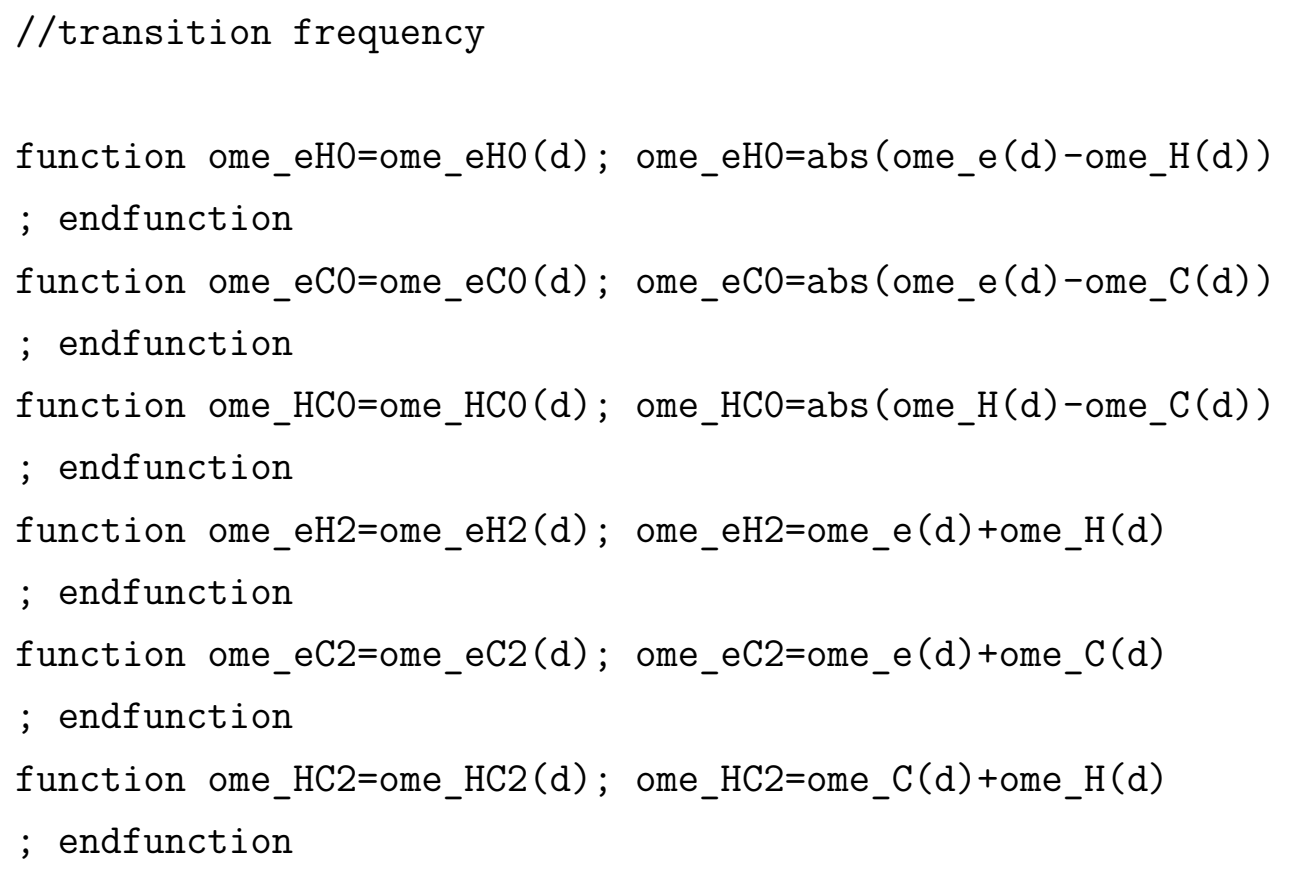


function $z c 1=z c 1(d) ; \quad z c 1=\left(2 * o m e \_C(d) * t a u_{-} D_{-} C(d)\right)-(1 / 2)$;

endfunction

function jtc1=jtc1(d); jtc1 $=(1+5 * z c 1(d) / 8+z c 1(d)-2 / 8) /(1+z c 1(d)$

$+z c 1(d) \wedge 2 / 2+z c 1(d) \wedge 3 / 6+4 * z c 1(d) \sim 4 / 81+z c 1(d) \wedge 5 / 81+z c 1(d)-6 / 648)$

; endfunction

//calc jt2

function $z h 2=z h 2(d) ; \quad z h 2=\left(2 * o m e \_e H 2(d) * t a u_{-} D_{-} H(d)\right)^{-}(1 / 2)$

; endfunction

function jth2 $=j \operatorname{th} 2(d) ; j \operatorname{th} 2=(1+5 * z h 2(d) / 8+z h 2(d)-2 / 8) /(1+z h 2(d)$

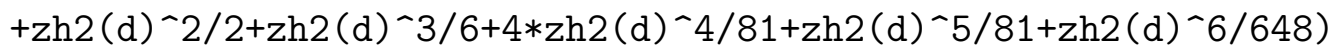

; endfunction

function $\mathrm{zc} 2=\mathrm{zc} 2(\mathrm{~d}) ; \mathrm{zc} 2=(2 *$ ome_eC2 $(d) *$ tau_D_C $(d))-(1 / 2)$

; endfunction

function jtc2 $=j t c 2(d) ; j$ tc $2=(1+5 * z c 2(d) / 8+z c 2(d)-2 / 8) /(1+z c 2(d)$

$+z c 2(d) \wedge 2 / 2+z c 2(d) \wedge 3 / 6+4 * z c 2(d) \wedge 4 / 81+z c 2(d)-5 / 81+z c 2(d)-6 / 648)$

; endfunction

//calc jro

function jreh0=jreho(d); jreh0=tau_rot (d)/(1+ome_eHO(d)^2

*tau_rot (d) 2 ); endfunction

function jrec0 $=j \operatorname{rec} 0(d) ; j r e c 0=t a u_{-} \operatorname{rot}(d) /\left(1+o m e \_e C 0(d) \wedge 2\right.$

*tau_rot(d)^2); endfunction

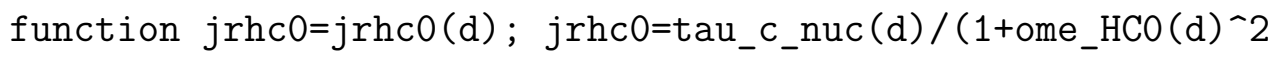

*tau_c_nuc(d)^2); endfunction

//calc jr1

function jreh1=jreh1(d); jreh1=tau_rot(d)/(1+ome_H(d)^2

*tau_rot(d)^2); endfunction

function jrec1=jrec1(d); jrec1=tau_rot (d)/(1+ome_C(d)^2

*tau_rot (d)`2); endfunction

function jrh1=jrh1(d); jrh1=tau_c_nuc(d)/(1+ome_H(d)^2

*tau_c_nuc(d)^2); endfunction

function jrc1=jrc1(d); jrc1=tau_c_nuc(d)/(1+ome_C(d)^2 
*tau_c_nuc(d)^2); endfunction

//calc jr2

function jreh2=jreh2(d); jreh2=tau_rot(d)/(1+ome_eH2(d)^2

*tau_rot (d) -2); endfunction

function jrec2=jrec2(d); jrec2=tau_rot (d)/(1+ome_eC2(d) ^2

*tau_rot (d)-2); endfunction

function jrhc2=jrhc2(d); jrhc2=tau_c_nuc(d)/(1+ome_HC2(d)^2

*tau_c_nuc(d)-2); endfunction

//wt = transition probebility of translatioavol motion

//two spin proton-electron and carbon electron

function $w \operatorname{th} 0=w \operatorname{th} 0(d)$; wthO $=\left(k_{-} t_{-} e H(d) * j \operatorname{th} 0(d)\right)$; endfunction

function wth1 $=$ wth $1(d)$; wth $1=3 / 2 * k_{-} t_{-} e H(d) * j \operatorname{th} 1(d)$; endfunction

function wth $2=$ wth $2(d)$; wth2 $=6 * k_{-} t_{-} e H(d) * j \operatorname{th} 2(d)$; endfunction

function wtc $0=w t c 0(d)$; wtc $0=\left(k_{-} t \_e C(d) * j t c 0(d)\right)$; endfunction

function wtc1=wtc1(d); wtc1 $=3 / 2 * k_{-} t_{-} e C(d) * j t c 1(d)$; endfunction

function wtc $2=w t c 2(d)$; wtc $2=6 * k_{-} t_{-} e C(d) * j t c 2(d)$; endfunction

//wr = transition probebility of rotatioavol motion

function wreh0 $=$ wreh0 $(d)$; wreh0 $=\left(k_{-} r_{-} e H(d) * j r e h 0(d)\right)$; endfunction function wreh1=wreh1(d); wreh1=3/2*k_r_eH(d)*jreh1(d); endfunction function wreh2 $=$ wreh2 $(d)$; wreh2 $=6 * k_{-} r_{-} e H(d) * j r e h 2(d)$; endfunction

function $\operatorname{wrec} 0=\operatorname{wrec} 0(d) ; \operatorname{wrec} 0=\left(k_{-} r_{-} e C(d) * j r e c 0(d)\right)$; endfunction function wrec1=wrec1(d); wrec1=3/2*k_r_eC $(d) * j r e c 1(d)$; endfunction function wrec $2=$ wrec2 $(d)$; wrec $2=6 * k_{-} r_{-} e C(d) * j r e c 2(d)$; endfunction

function $\operatorname{wrhc} 0=\operatorname{wrhcO}(d)$; $\operatorname{wrhcO}=\left(k_{-} r_{-} H C(d) * j r h c 0(d)\right)$; endfunction function $\operatorname{wrh} 1=w r h 1(d)$; wrh1=3/2*k_r_HC (d)*jrh1(d); endfunction function $\operatorname{wrc1}=\operatorname{wrc1}(d)$; $\operatorname{wrc1} 1=3 / 2 * \mathrm{k}_{-} r_{-} \mathrm{HC}(d) * j r c 1(d)$; endfunction function wrhc2=wrhc2 $(d)$; wrhc2 $=6 * \mathrm{k}_{-}{ }_{-} \_\mathrm{HC}(\mathrm{d}) * \mathrm{jrhc} 2(\mathrm{~d})$; endfunction 
function sig_eH=sig_eH(d); sig_eH=wth2(d)-wth0 (d)

+wreh2(d)-wreh0 (d); endfunction

function sig_eC=sig_eC (d); sig_eC=wtc2 (d) -wtc0 (d)

+wrec2(d)-wrec0(d); endfunction

function sig_HC=sig_HC(d); $\operatorname{sig\_ HC}=($ wrhc2 $(d)-w r h c 0(d))$

; endfunction

function rho_H=rho_H(d); rho_H= (wth2 (d)+2*wth1 (d)

+wth0 (d)+wreh2 (d) +2*wreh1 (d) +wreh0 (d)+wrhc2 (d)

$+2 * w r h 1(d)+w r h c 0(d))$; endfunction

function rho_C=rho_C $(d)$; rho_ $C=($ wtc2 $(d)+2 *$ wtc1 $(d)$

+ wtc0 $(d)+$ wrec2 $(d)+2 * w r e c 1(d)+w r e c 0(d)+w r h c 2(d)$

$+2 * w r c 1(d)+w r h c 0(d))$; endfunction

function rho_HwoC=rho_HwoC $(d)$; rho_HwoC= $($ wth2 $(d)$

$+2 * w \operatorname{th} 1(d)+w \operatorname{th} 0(d)+$ wreh2 (d) +2*wreh1 (d) +wreh0 (d))

; endfunction

function $x i_{-} \mathrm{HC}_{-} \mathrm{H}=\mathrm{xi} \mathrm{HC}_{-} \mathrm{H}(\mathrm{d})$; $\mathrm{xi} \mathrm{H}_{-} \mathrm{HC} \mathrm{H}=(\mathrm{sig}$ _eH (d)

*rho_C (d)-sig_eC (d)*sig_HC(d))/(rho_H(d)*rho_C (d)

-sig_HC(d)^2); endfunction

function $\mathrm{xi}$ _HC_C $=\mathrm{xi} \mathrm{HC}_{-} \mathrm{C}(\mathrm{d})$; $\mathrm{xi} \mathrm{H}_{-} \mathrm{HC} \mathrm{C}=(\mathrm{sig}$ _eC $(\mathrm{d})$

*rho_H(d)-sig_eH(d)*sig_HC(d))/(rho_H(d)*rho_C (d)

-sig_HC(d)-2); endfunction

function $\mathrm{xi} H=\mathrm{xi} H(\mathrm{~d})$; $\mathrm{xi}+\mathrm{H}=($ wth2 $(\mathrm{d})-\mathrm{wth} 0(\mathrm{~d})+$ wreh2 $(d)$

-wreh0 (d)) / (wth2 (d)+2*wth1 (d)+wth0 (d)+wreh2 (d)

$+2 *$ wreh $1(d)+$ wreho $(d))$; endfunction 
function $\mathrm{xi} C=\mathrm{xi} C(\mathrm{~d}) ; \mathrm{xi} C=($ wtc2 $(d)-w \operatorname{tc} 0(d)+$ wrec2 $(d)$

$-\operatorname{wrec} 0(d)) /(\operatorname{wtc} 2(d)+2 * w t c 1(d)+w t c 0(d)+w r e c 2(d)$

$+2 * \operatorname{wrec} 1(d)+\operatorname{wrec} 0(d))$; endfunction

function $x i_{-} H C=x i_{-} H C(d) ; x i \_H C=(w r h c 2(d)-w r h c 0(d)) /($ wrhc2 $(d)$

$+2 * \operatorname{wrc} 1(d)+\operatorname{wrhc} 0(d))$; endfunction

function eps_C=eps_C(d); eps_C=(1-xi_C(d)

$*($ ge*mub/hbar)/gam_C) /(14.1/B_O(d)); endfunction

function eps_HC_C=eps_HC_C $(d)$; eps_HC_C=(1-xi_HC_C (d)

*(ge*mub/hbar)/gam_C)/(14.1/B_O(d)); endfunction

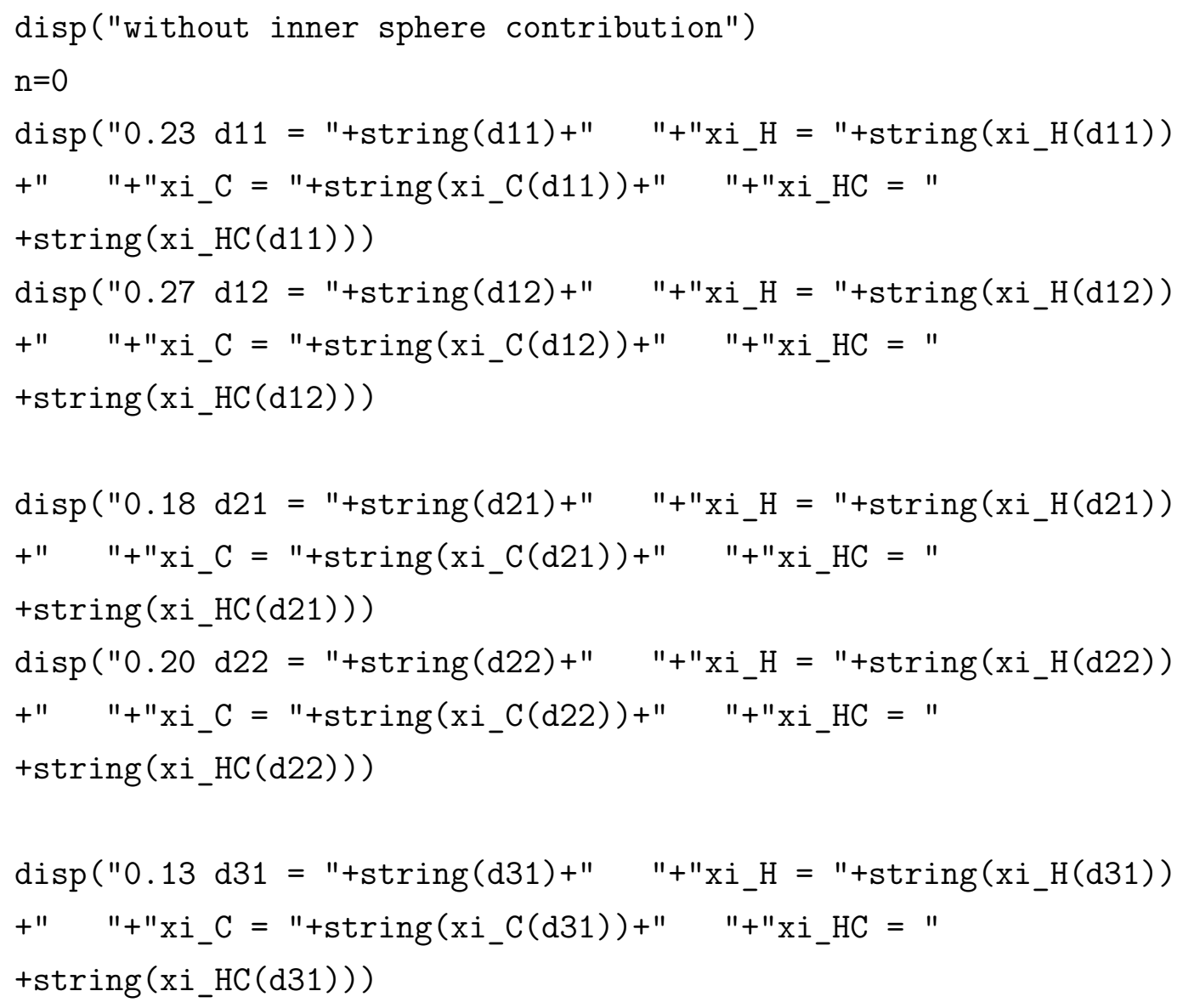




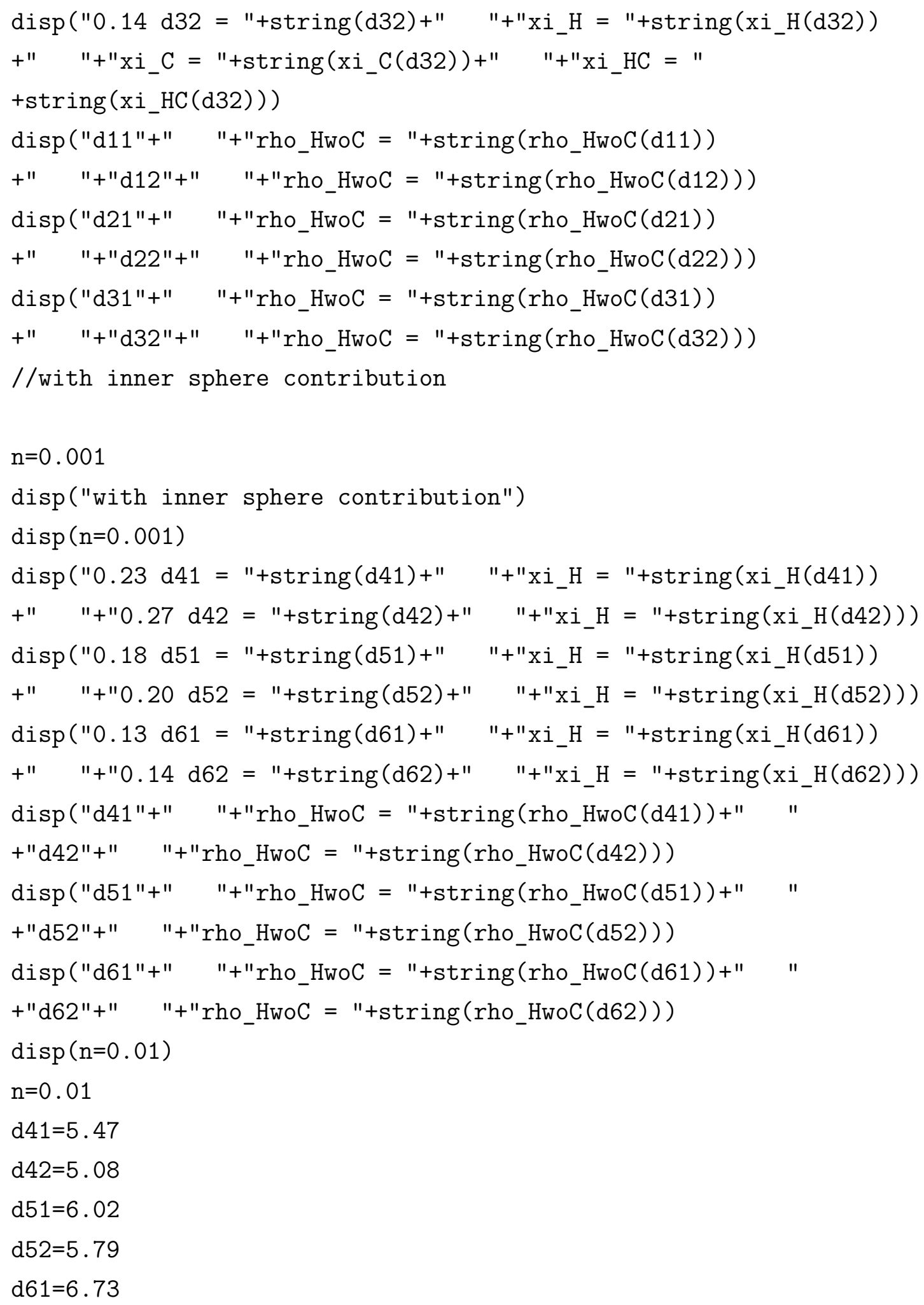


$\mathrm{d} 62=6.57$

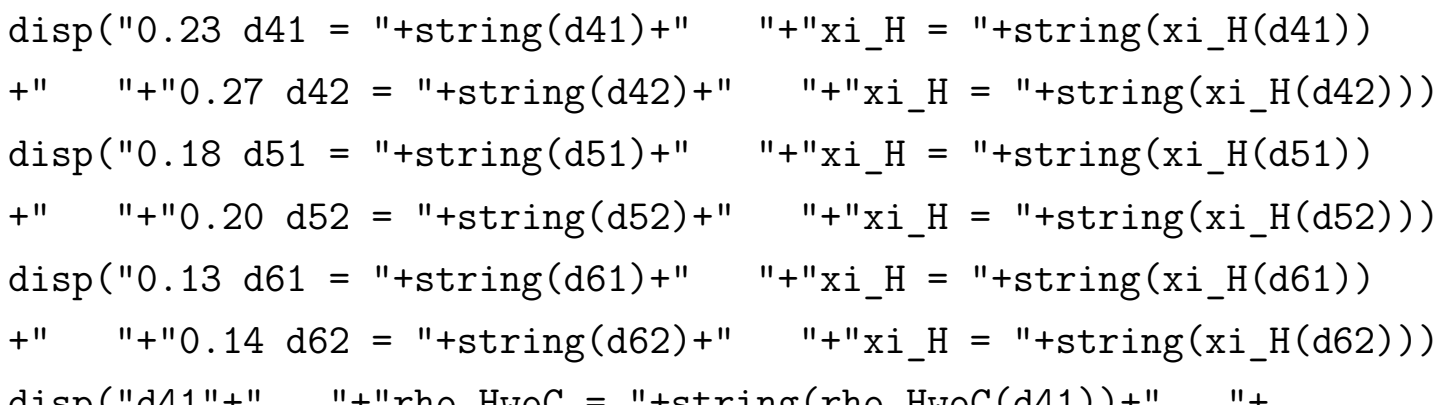

disp("d41"+" "+"rho_HwoC = "+string (rho_HwoC $(d 41))+"$ "+

"d42"+" "+"rho_HwoC = "+string $\left.\left(r h o \_H w o C(d 42)\right)\right)$

disp ("d51"+" "+"rho_HwoC = "+string (rho_HwoC(d51))+" "+

"d52"+" "+"rho_HwoC = "+string $($ rho_HwoC (d52)))

$\operatorname{disp}(" d 61 "+"$ "+"rho_HwoC = "+string (rho_HwoC $(\mathrm{d} 61))+"$ "+

"d62"+" "+"rho_HwoC = "+string $($ rho_HwoC $(d 62)))$

$\operatorname{disp}(\mathrm{n}=0.0001)$

$\mathrm{n}=0.0001$

$\mathrm{d} 41=3.62$

$\mathrm{d} 42=3.19$

$\mathrm{d} 51=4.23$

$\mathrm{d} 52=3.97$

$\mathrm{d} 61=5.02$

$\mathrm{d} 62=4.84$

$\operatorname{disp}(" 0.23 \mathrm{~d} 41=$ = +string $(\mathrm{d} 41)+" \quad$ "+"xi_H = "+string $(\mathrm{xi} H(\mathrm{~d} 41))$

+" "+"0.27 d42 = "+string (d42)+" "+"xi_H = "+string $\left.\left(\mathrm{xi}_{-} H(\mathrm{~d} 42)\right)\right)$

$\operatorname{disp}(" 0.18 \mathrm{~d} 51$ = "+string $(\mathrm{d} 51)+"$ "+"xi_H = "+string $(\mathrm{xi} H(\mathrm{~d} 51))$

+" "+"0.20 d52 = "+string (d52)+" "+"xi_H = "+string $\left.\left(\mathrm{xi}_{-} H(\mathrm{~d} 52)\right)\right)$

$\operatorname{disp}\left(" 0.13 \mathrm{~d} 61=\right.$ = +string $(\mathrm{d} 61)+" \quad$ "+"xi_H = "+string $\left(\mathrm{xi} \mathrm{H}_{-} \mathrm{H}(\mathrm{d} 61)\right)$

+" "+"0.14 d62 = "+string (d62)+" "+"xi_H = "+string $\left.\left(\mathrm{xi}_{-} H(\mathrm{~d} 62)\right)\right)$

disp("d41"+" "+"rho_HwoC = "+string (rho_HwoC $(\mathrm{d} 41))+$ " "

+"d42"+" "+"rho_HwoC = "+string $($ rho_HwoC $(d 42)))$

$\operatorname{disp}(" \mathrm{~d} 51 "+" \quad$ "+"rho_HwoC = "+string (rho_HwoC(d51))+" "

+"d52"+" "+"rho_HwoC = "+string (rho_HwoC(d52)))

disp("d61"+" "+"rho_HwoC = "+string (rho_HwoC $(\mathrm{d} 61))+"$ " 
+"d62"+" "+"rho_HwoC = "+string $($ rho_HwoC $(d 62)))$

disp ("carbon enhancement")

$\mathrm{n}=0$

$\operatorname{disp}(" 0.23 \mathrm{~d} 11=$ "+string $(\mathrm{d} 11)+" \quad$ "+"eps_C $=$ "

+string (eps_C (d11))+" "+"0.27 d12 = "+string(d12)+" "

+"eps_C = "+string (eps_C(d12)))

$\operatorname{disp}(" 0.18 \mathrm{~d} 21=$ "+string $(\mathrm{d} 21)+" \quad$ "+"eps_C $=$ "

+string (eps_C $(\mathrm{d} 21))+" \quad$ "+"0.20 d22 = "+string(d22)+" "

+"eps_C = "+string $($ eps_C $(d 22)))$

$\operatorname{disp}(" 0.13 \mathrm{~d} 31=$ = +string $(\mathrm{d} 31)+" \quad$ "+"eps_C $=$ "

+string (eps_C (d31))+" "+"0.14 d32 = "+string(d32)+" "

+"eps_C = "+string $($ eps_C $($ d32) $))$

disp("3 spin system couplin factor for C")

$\mathrm{n}=0$

$\operatorname{disp}(" 0.23 \mathrm{~d} 11=$ = +string $(\mathrm{d} 11)+" \quad$ "+"xi_HC_C = "

+string (xi_HC_C (d11))+" "+"0.27 d12 = "+string (d12)+" "

$+" x i_{-} H C_{-} C=$ "string $\left(x_{-}\right.$HC_C $\left.\left._{-}(\mathrm{d} 12)\right)\right)$

$\operatorname{disp}(" 0.18 \mathrm{~d} 21=$ "+string $(\mathrm{d} 21)+" \quad$ "+xi_HC_C $=$ "

+string $\left(x_{-}\right.$HC_C $\left._{-}(\mathrm{d} 21)\right)+"$ "+"0.20 d22 = "+string $(\mathrm{d} 22)+"$ "

$+" \mathrm{xi} \mathrm{HC}_{-} \mathrm{C}=$ "+string $\left.\left(\mathrm{xi} \mathrm{H}_{-} \mathrm{HC} \mathrm{C}(\mathrm{d} 22)\right)\right)$

$\operatorname{disp}(" 0.13 \mathrm{~d} 31=$ "+string $(\mathrm{d} 31)+" \quad$ "+"xi_HC_C $=$ "

+string $\left(x_{-}\right.$HC_C $\left._{-}(\mathrm{d} 31)\right)+"$ "+"0.14 d32 = "+string $(\mathrm{d} 32)+"$ "

+"xi_HC_C = "+string $\left.\left(\mathrm{xi}_{-} \mathrm{HC}_{-} \mathrm{C}(\mathrm{d} 32)\right)\right)$

disp("3 spin system carbon enhancement")

$\mathrm{n}=0$ 


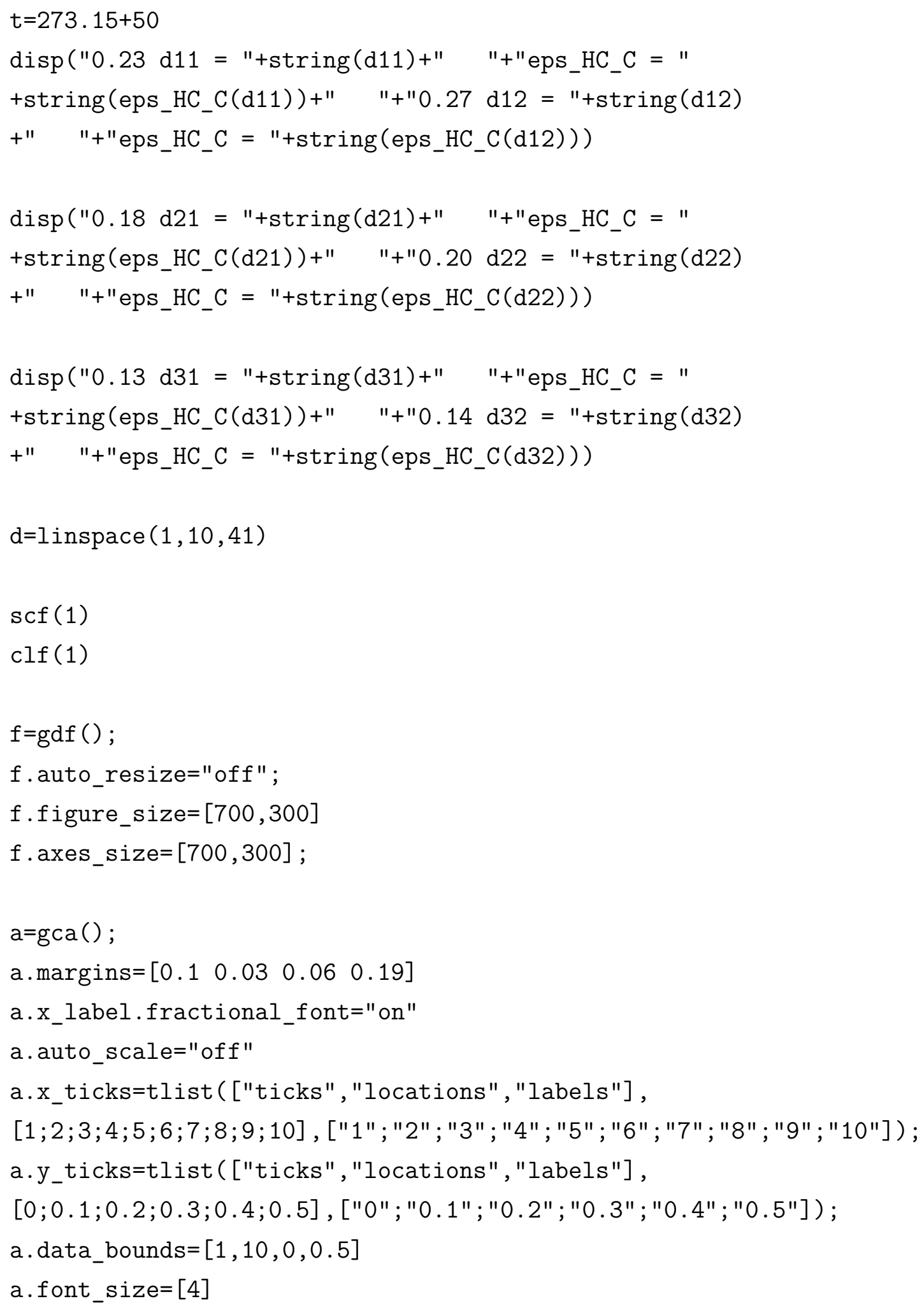




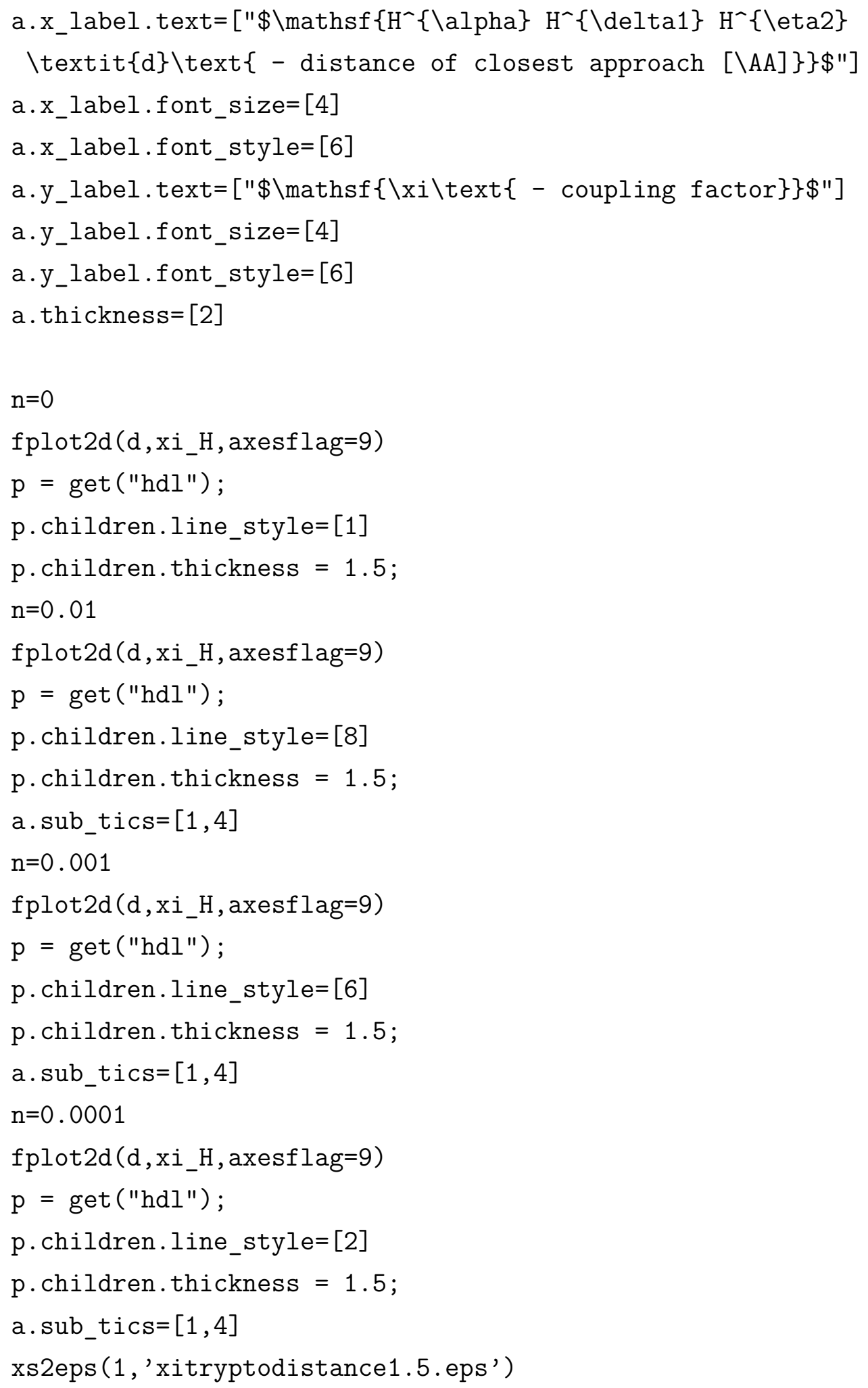




\section{C.3. NMRPipe scripts}

NMRPipe script for the Fourier transformation of the direct dimension:

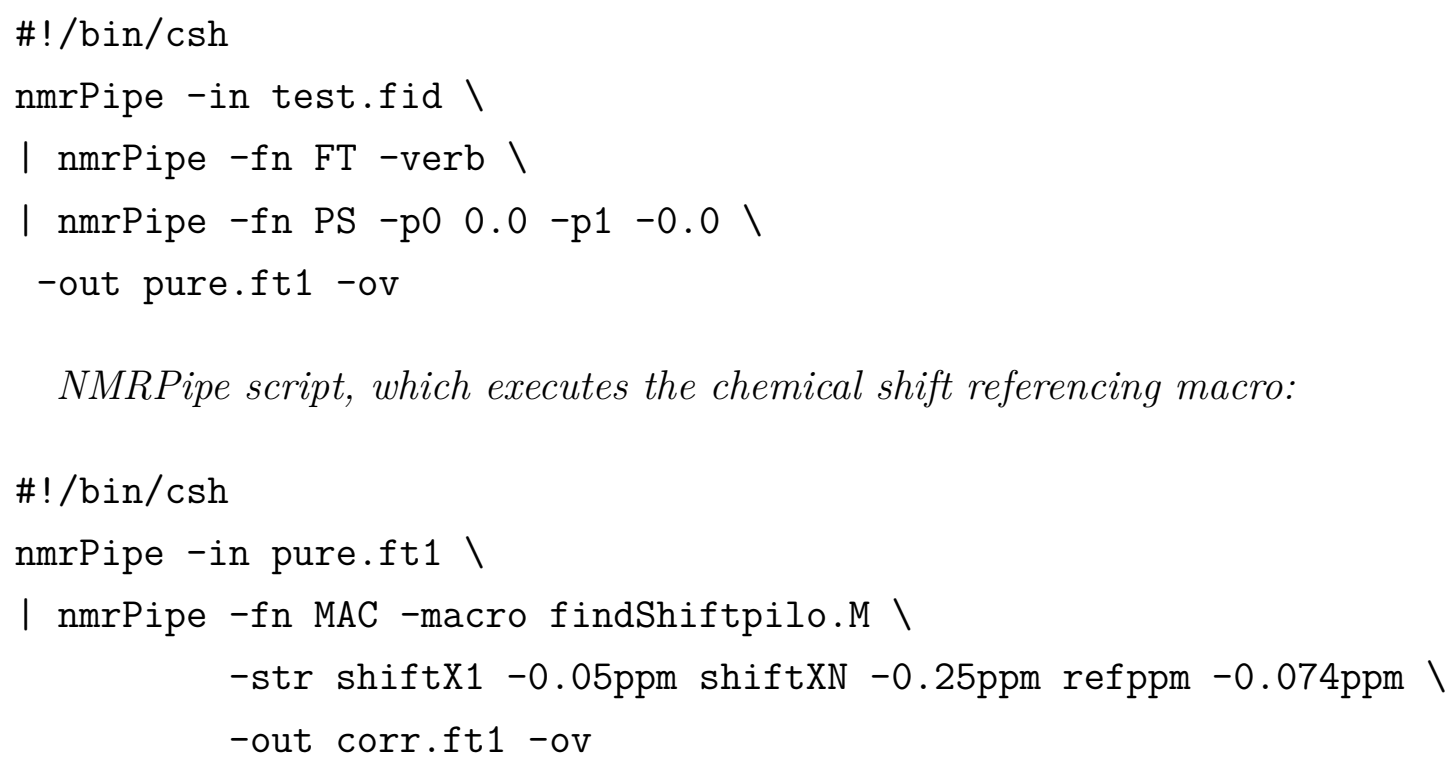

NMRPipe macro for the chemical shift referencing (findShiftpilo.M):

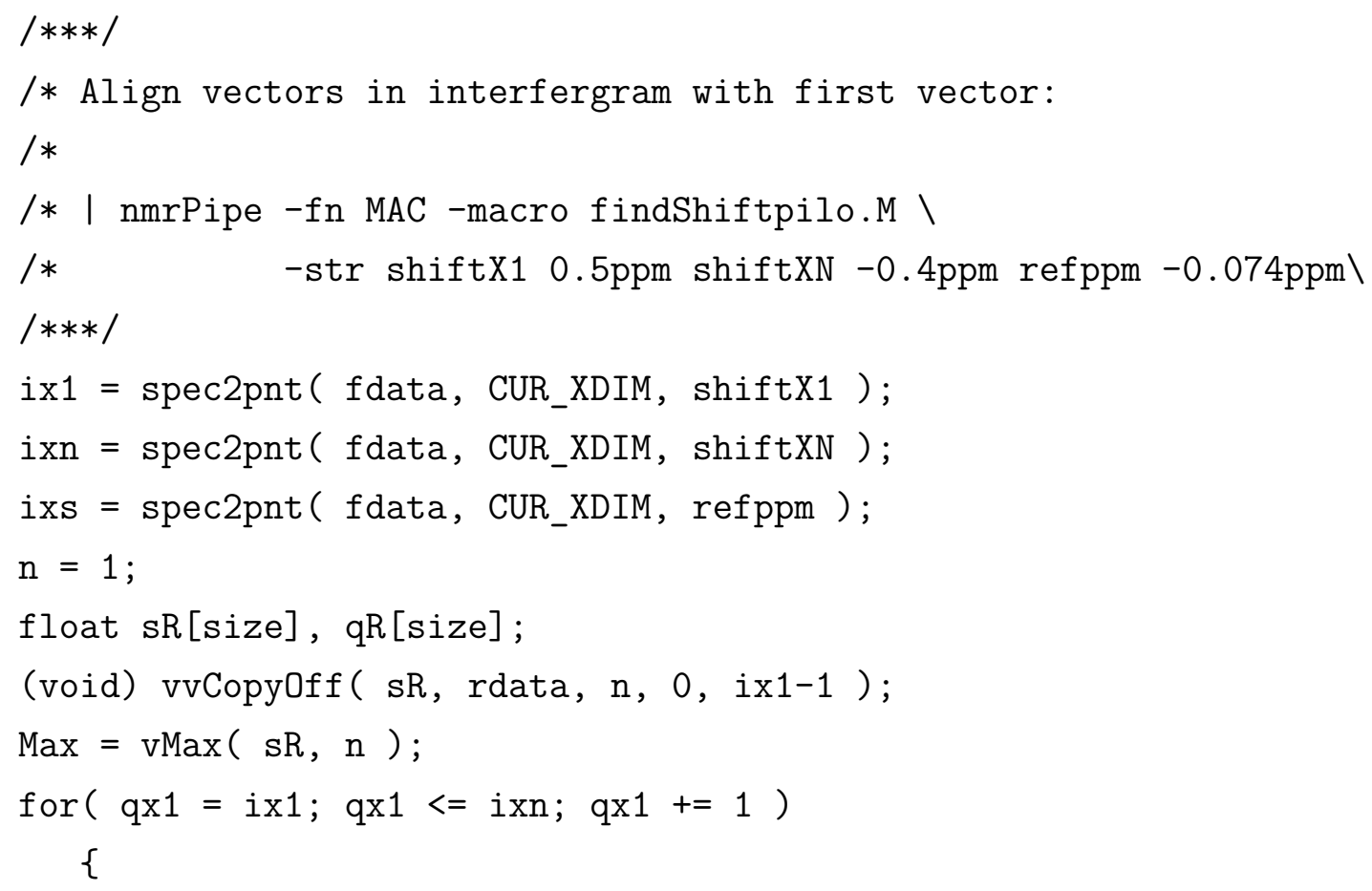




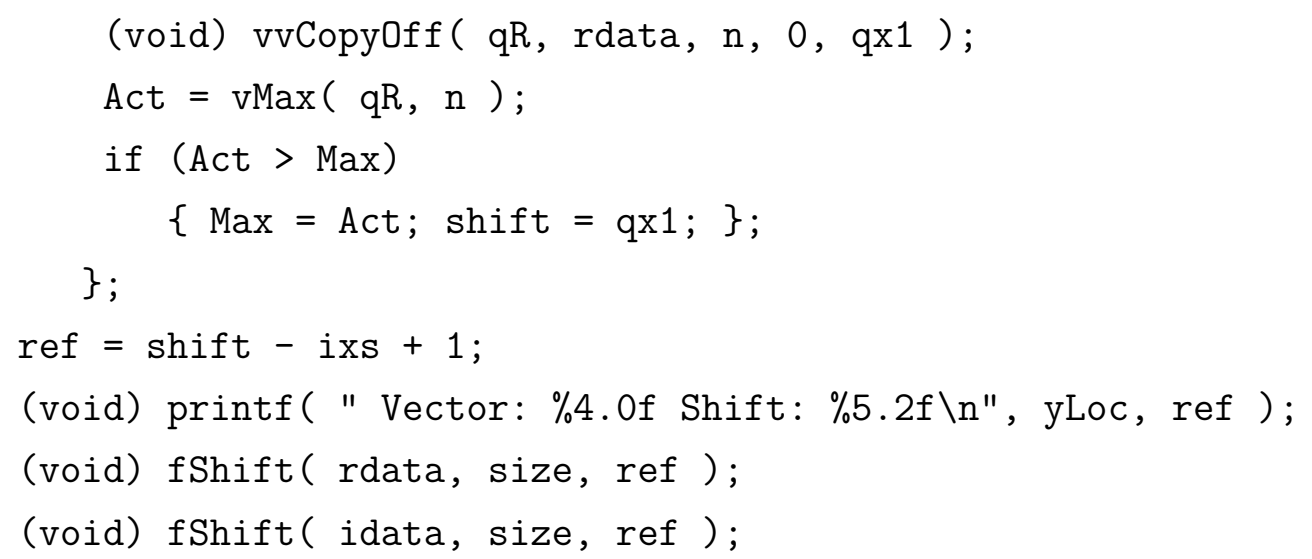

NMRPipe script for the inverse Fourier transformation and the concluding script for the processing of the two dimensional data set:

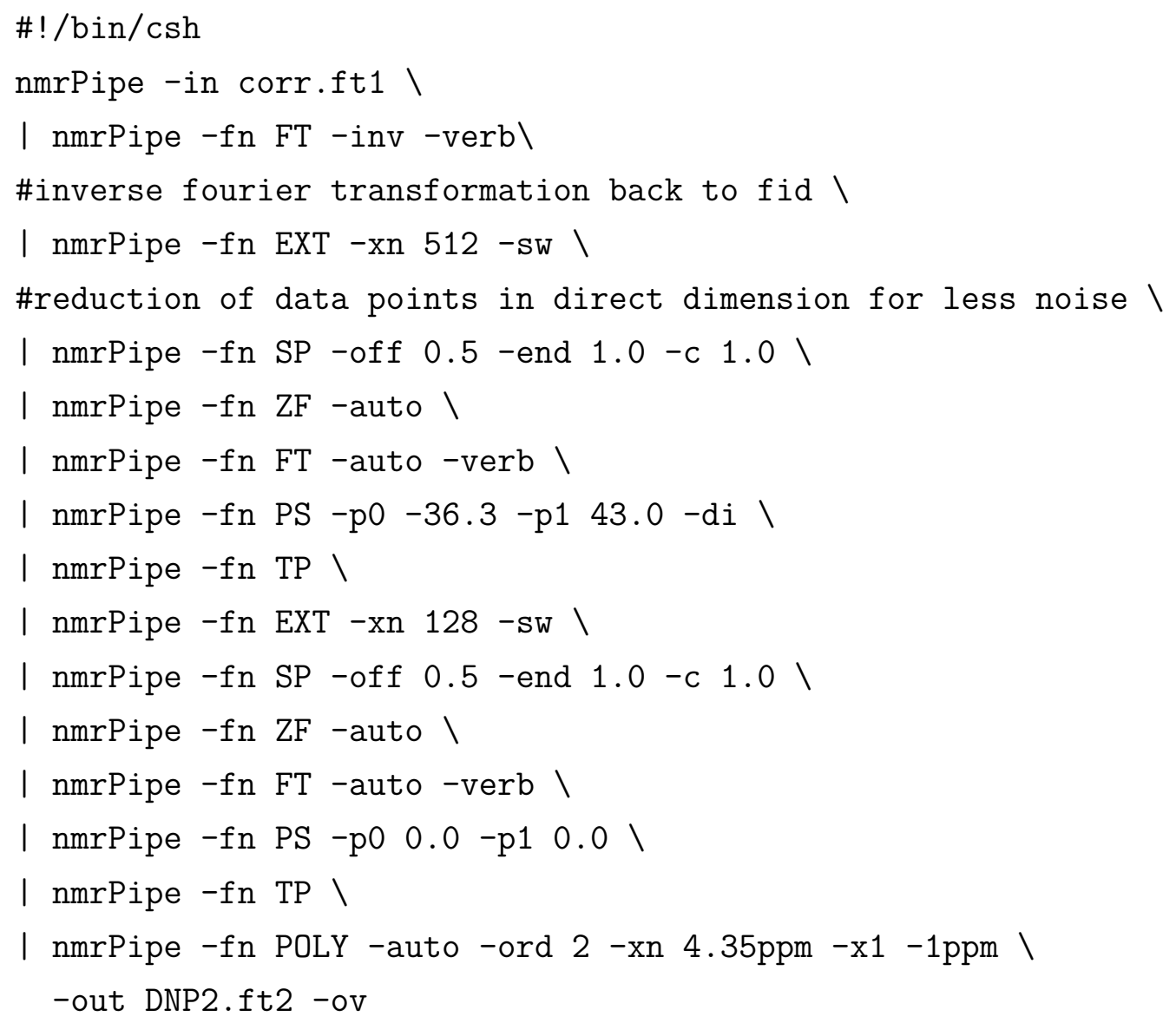





\section{Curriculum vitae}

\section{Personal data}

\begin{tabular}{ll}
\hline \hline Name & Philip Lottmann \\
Birthday & 21.01 .1986 \\
City of birth & Hildesheim \\
Citizenship & German
\end{tabular}

School and academic education

\begin{tabular}{ll}
\hline 2005 & University entrance certificate (German Abitur) at the \\
& Roswitha Gymnasium, Bad Gandersheim, Germany \\
& Studies of chemistry at the Georg August University Göt- \\
& tingen, Germany \\
& Internship at Sanofi Aventis: CAS - Structural biology, \\
& Frankfurt, Germany \\
& Safed Summer School on "Dynamic Nuclear Polarization \\
& at high magnetic fields - theory and applications", Safed, \\
& Israel \\
& Diploma in Chemistry, thesis: "Measurements on and \\
& with a DNP-Shuttle-Spectrometer" \\
& EMBO Practical Course on "Structure, dynamics and \\
& function of biomacromolecules by solution NMR", Mu- \\
& nich, Germany \\
& PhD student at Max Planck Institute for Biophysical \\
& Chemistry / Georg August University Göttingen, Ger- \\
& many, thesis title: "Sensitivity Enhanced NMR", princi- \\
& ple investigator Prof. Dr. C. Griesinger \\
since 07.2010 &
\end{tabular}




\section{Awards and Scholarships}

\begin{tabular}{ll}
\hline \hline 12.2007 & Otto-Wallach-Prize for outstanding performances in the \\
& intermediate examination \\
$03.2011-02.2013 \quad$ & Scholarship of the fund of the chemical industry \\
& "Chemiefonds-Stipendium der Stiftung Stipendien-Fonds \\
& des Verbandes der Chemischen Industrie"
\end{tabular}

\section{Publications}

A. Krahn, P. Lottmann, T. Marquardsen, A. Tavernier, M.-T. Tuerke, M. Reese, A. Leonov, M. Bennati, P. Hoefer, F. Engelke and C. Griesinger, Shuttle DNP Spectrometer with a Two-Center Magnet, Phys. Chem. Chem. Phys., 2010, 12, 5830 .

P. Lottmann, T. Marquardsen, A. Krahn, A. Tavernier, P. Hoefer, M. Bennati, F. Engelke and C. Griesinger, Evaluation of a Shuttle DNP Spectrometer by Calculating the Coupling and Global Enhancement Factors of L-Tryptophan, Appl. Magn. Reson., 2012, 43, 207.

Göttingen, December 2013 

